\title{
A Chemoproteomic Method for Profiling Inhibitor-Bound Kinase Complexes
}

\author{
Linglan Fang ${ }^{\dagger}$, Sujata Chakraborty ${ }^{\dagger}$, Emily M. Dieter ${ }^{\dagger}$, Zachary E. Potter ${ }^{\dagger}$, Chloe K. Lombard ${ }^{\dagger}$, \\ and Dustin J. Maly ${ }^{*} \dagger,+$ \\ ${ }^{\dagger}$ Department of Chemistry and ${ }^{\star}$ Biochemistry, University of Washington, Seattle, USA \\ *Email: djmaly@uw.edu
}

\section{Table of Contents:}

- Supporting Figures (Figure S1-S13)

- Materials and Reagents

- Generation of Stable HeLa Cell Line

- Synthesis and Characterization Data of 1-TCO, 2-TCO, 3-TCO

- Protein purification and kinase activity assay

- SH3 Domain Pull-Down Assay

- Generation of Tetrazine-Beads

- Procedure of Enriching Probe-Bound Target in Cell Lysates

- Procedure of Enriching probe-bound kinase complex from Live Cell

- Proteomic Data Acquisition, Process, and Visualization of Target Interactome

- Procedure of Clickable Proximity Ligation Assay

- Imaging Data Acquisition and Analysis

- References

- High-Resolution Mass Spectrometric (HRMS) Data

- NMR Spectra

- Original immunoblots 

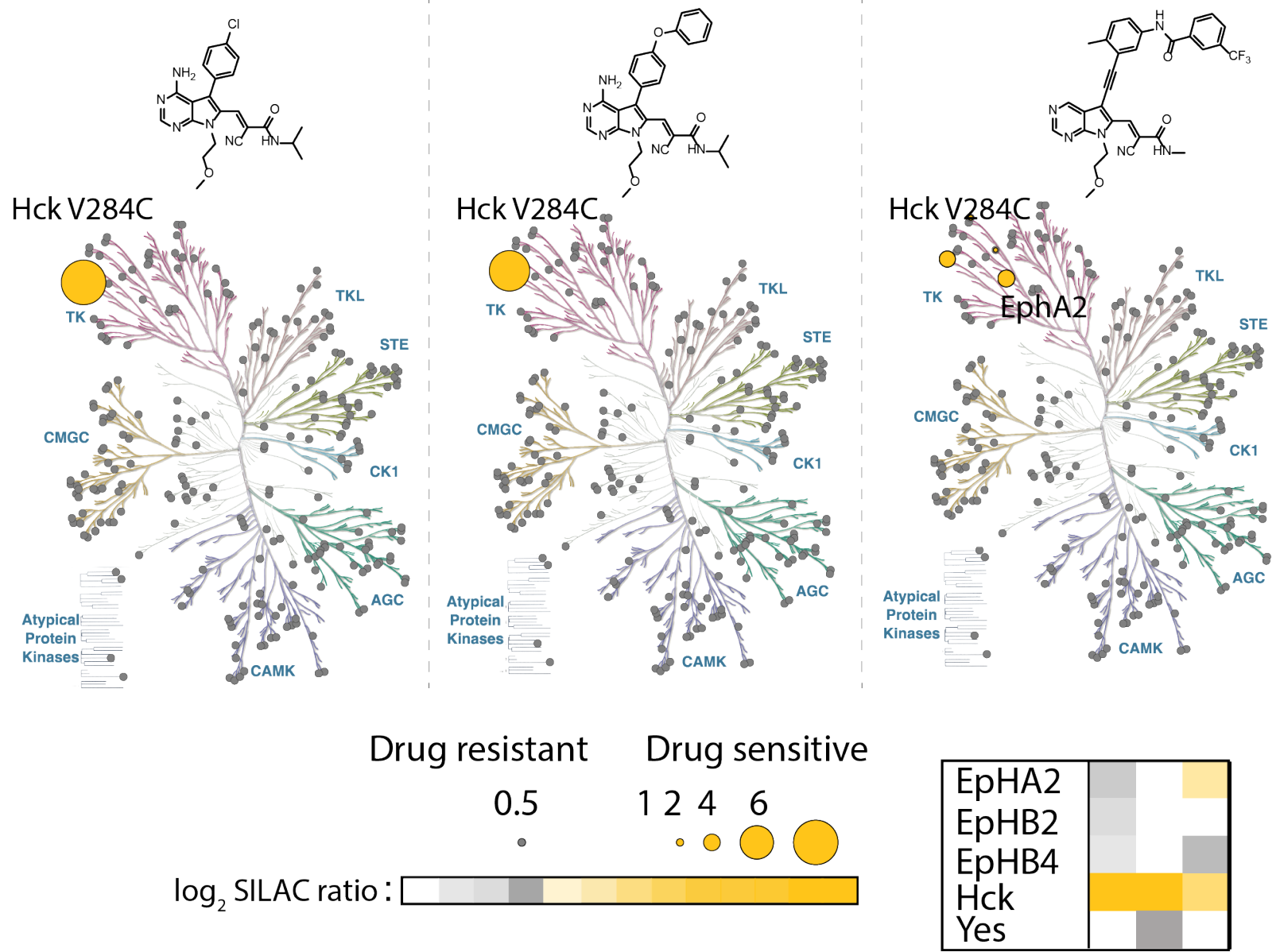

Figure S1. Phylogenetic trees showing the kinobead-based chemoproteomic profiling of CystIMATIK probes. Significantly competed kinases are shown as yellow circles with the size corresponding to the level of competition (larger circle, more competed). See the Supplementary Information of Mol. Cell 2019, 74, p. 393-408 for primary profiling data. 
A

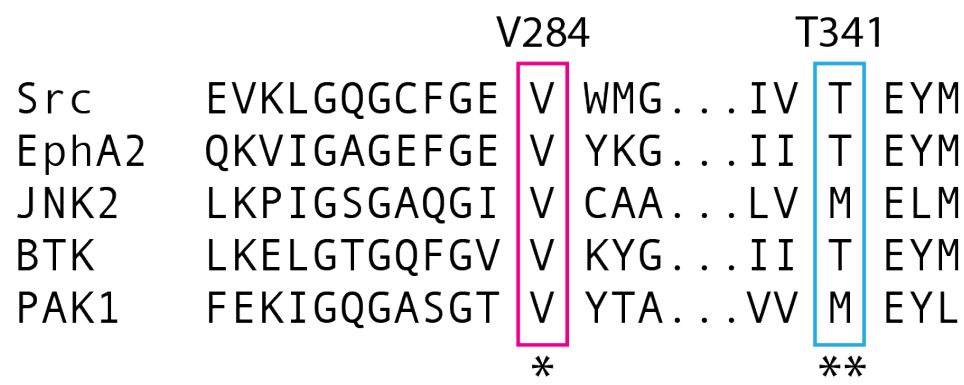

$\mathrm{B}$

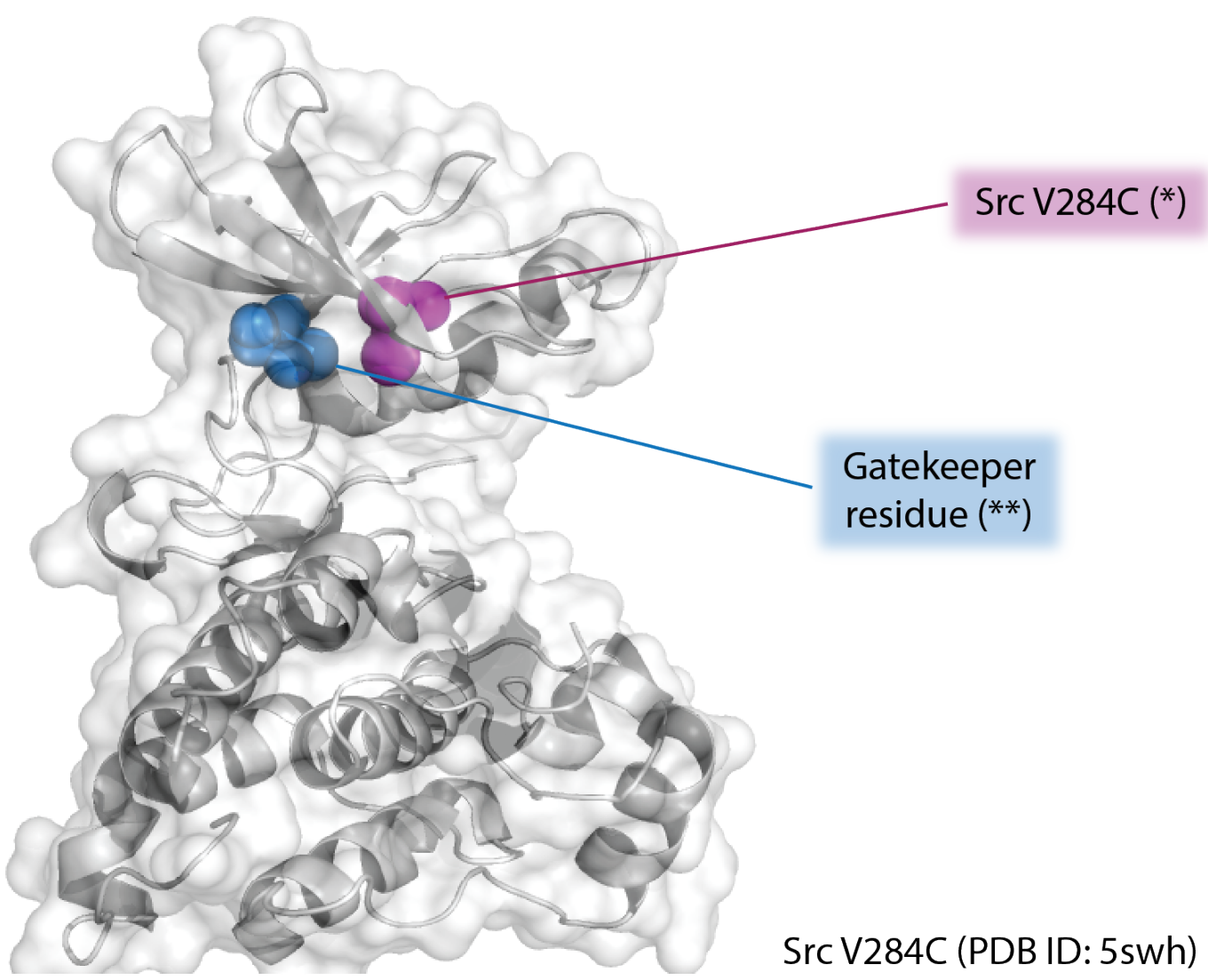

Figure S2. Sensitization of kinases to CystIMATIK probes. Introduction of a Cys at the residue homologous to V284 of Src and, if required, a Thr at the gatekeeper position sensitizes kinases to CystIMATIK probes. (A) Sequence alignment of the nonreceptor tyrosine kinases Src and BTK, the receptor tyrosine kinase Epha2, and the serine/threonine kinases PAK1 and JNK2. Residues at the position equivalent to V284 of Src are framed in magenta. Gatekeeper residues are framed in blue. Sequences used for sequence alignment are: PDB ID (Src): 2BDF; PDB ID (EphA2): 5I9Y; PDB ID (JNK2): 3E7O; PDB ID (BTK): 5FBO; PDB ID (PAK1): 2HY8. (B) X-ray crystal structure of the catalytic domain of Src V284C (PDB ID: 5SWH). The Cys284 residue is colored in magenta. The gatekeeper residue (T341) is colored in blue. 


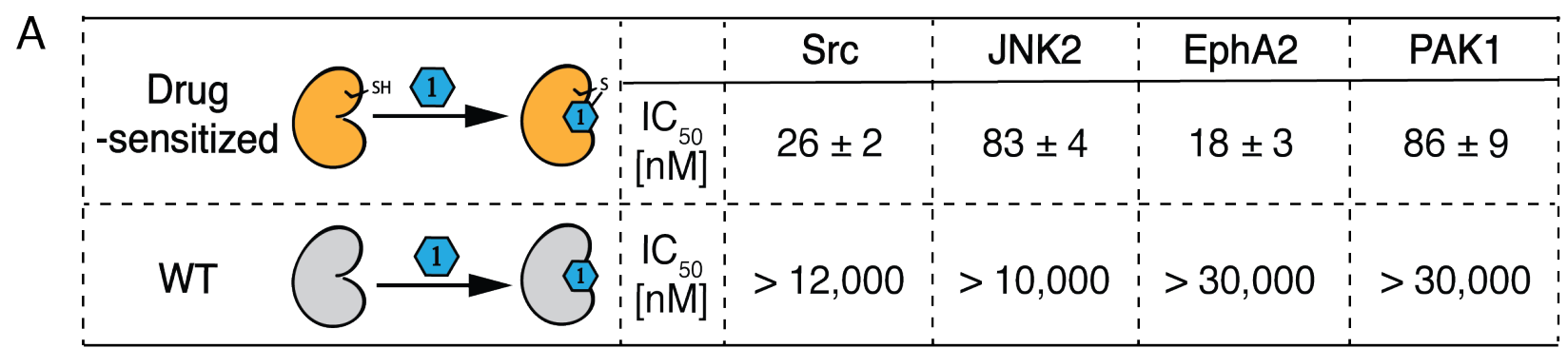

B
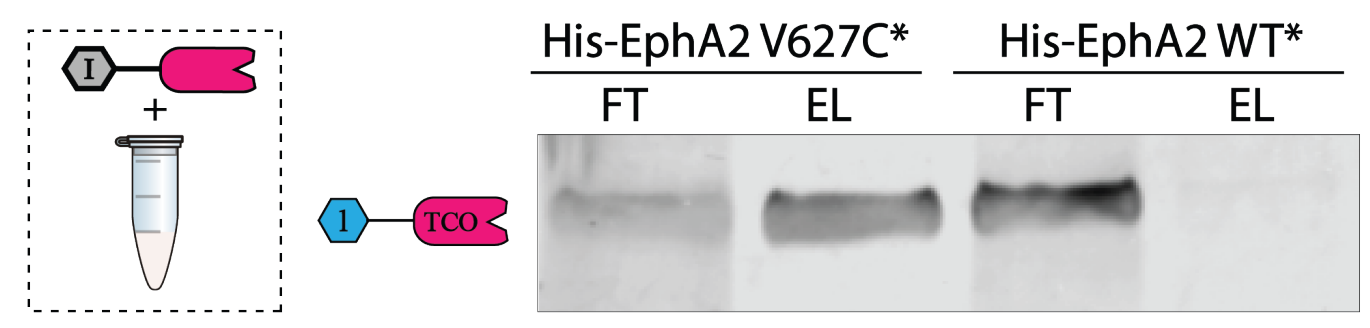

EphA2

C
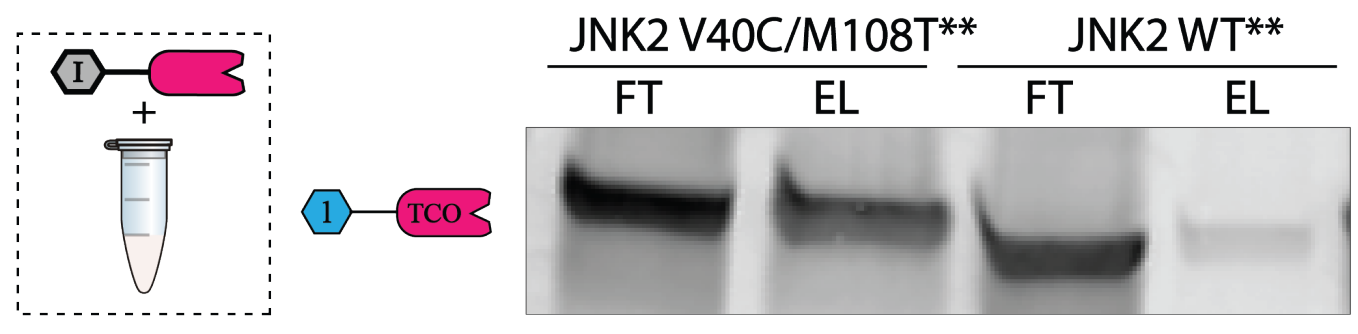

JNK2

D

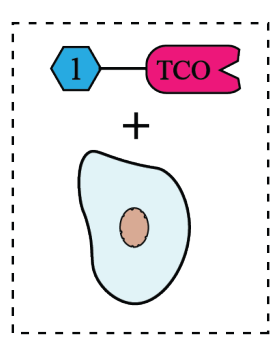

Mock Flag-BTK WT ${ }^{* * *}$ Flag-BTK V416C ${ }^{* * *}$

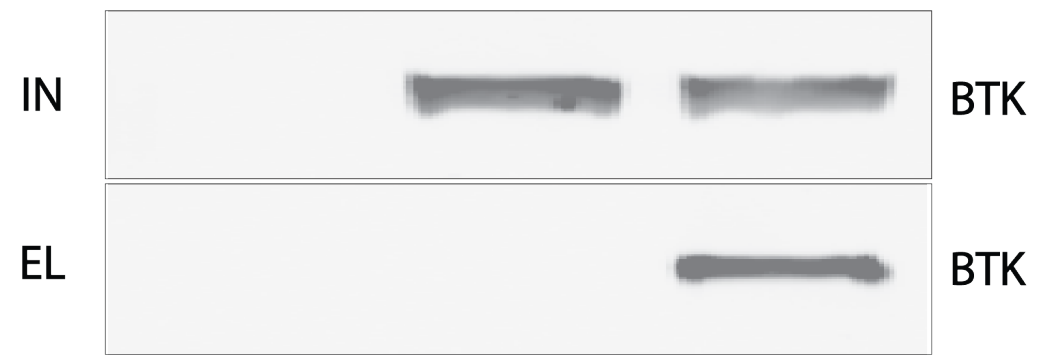

Notes:* N-terminal His-tagged recombinant EphA2 (residue 590 - 876); ** Full-length reco-mbinant JNK2; ***N-terminal Flag-tagged full-length BTK

Figure S3. Sensitization of diverse kinases to CystIMATIK probes $\mathbf{1}$ and 1-TCO. (A) $\mathrm{IC}_{50}$ values of 1 against recombinant Src V284C, JNK2 V40C/M108T, EphA2 V627C and PAK1 $\mathrm{V} 286 \mathrm{C} / \mathrm{M} 346 \mathrm{~T}$, and wild-type Src, JNK2, EphA2 and PAK1. IC $\mathrm{I}_{50}$ values shown are mean $\pm \mathrm{SEM}$, $n=3$. (B) 1-TCO selectively enriches drug-sensitized EphA2 (V627C; residue 590 - 876) from cell lysates. HEK293 cell lysate containing either N-terminal His-tagged EphA2 V627C or EphA2 WT were treated with 1-TCO $(10 \mu \mathrm{M})$, incubated with tetrazine-conjugated sepharose beads (Tzbeads), and captured proteins were eluted under reducing and denaturing conditions. An anti-His Tag immunoblot of the flow through (FT) and elution (EL) are shown. (C) 1-TCO selectively enriches drug-sensitized JNK2 (V40C/M108T) from cell lysates. HEK293 cell lysate containing either JNK2 V40C/M108T or JNK2 WT were treated with 1-TCO $(10 \mu \mathrm{M})$, incubated with Tzbeads, and captured proteins were eluted under reducing and denaturing conditions. An anti-JNK2 
immunoblot of the flow through (FT) and elution (EL) are shown. (D) 1-TCO selectively enriches drug-sensitized BTK (V416C) from cells. HEK293 cells transiently expressing either N-terminal Flag-tagged BTK (V416C) or BTK WT were treated with 1-TCO $(2.5 \mu \mathrm{M})$, lysed, and then incubated with Tz-beads. Captured proteins were eluted under reducing and denaturing conditions. An anti-DYKDDDDK Tag (Flag Tag) immunoblot of the input (IN) and elution (EL) are shown. Note: in Figure S3D, elution samples were 2-fold more concentrated than input samples. 
A

\begin{tabular}{|c|c|c|c|c|}
\hline & Src & JNK2 & EphA2 & PAK1 \\
\hline & $28 \pm 3$ & $180 \pm 10$ & $300 \pm 30$ & $151 \pm 4$ \\
\hline WT & $>12,000$ & $>10,000$ & $>30,000$ & $>30,000$ \\
\hline
\end{tabular}

$\mathrm{B}$

\begin{tabular}{|c|c|c|c|c|}
\hline & Src & JNK2 & EphA2 & PAK1 \\
\hline & $39 \pm 3$ & $6.2 \pm 0.2$ & $32 \pm 2$ & $>30,000$ \\
\hline WT & $>12,000$ & $>10,000$ & $890 \pm 60$ & $>30,000$ \\
\hline
\end{tabular}

C

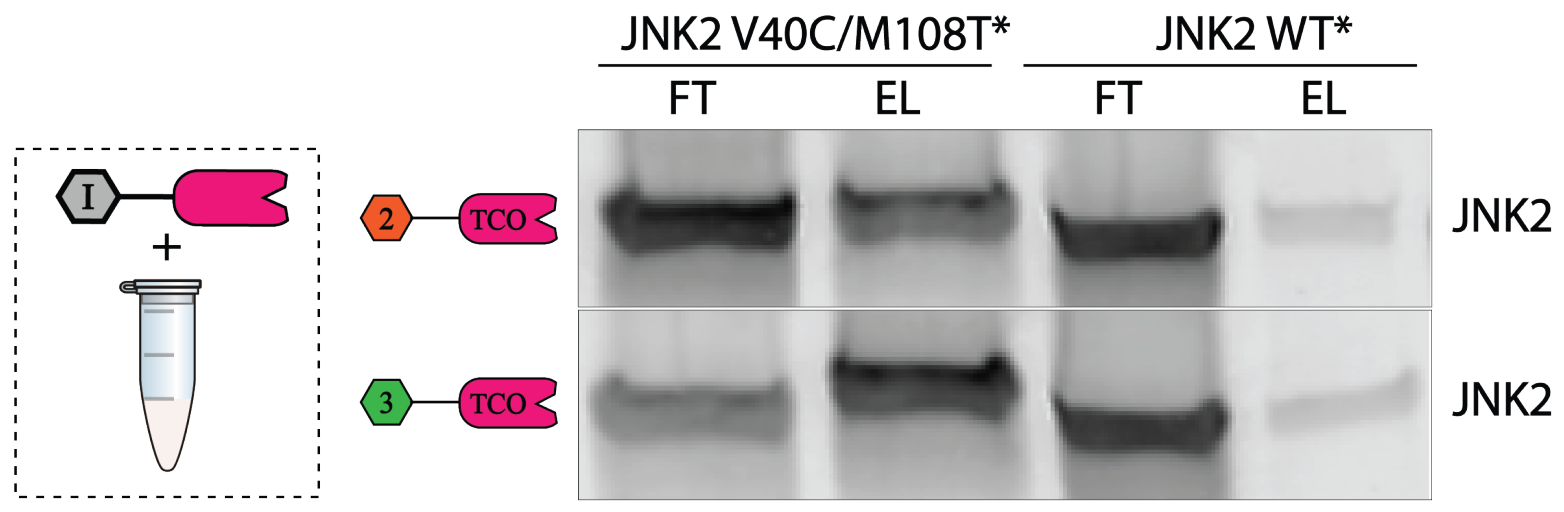

$\mathrm{D}$

Mock Flag-BTK WT** Flag-BTK V416C**
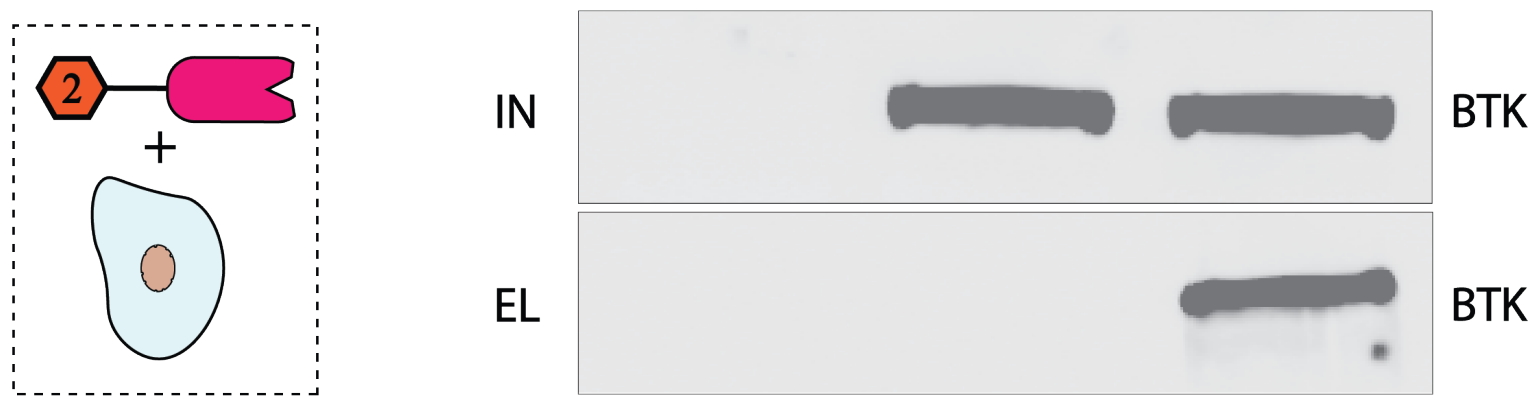

Notes: *** Full-length recombinant JNK2; ** N-terminal Flag-tagged full-length BTK

Figure S4. Sensitization of diverse kinases to conformation-selective CystIMATIK probes. (A) $\mathrm{IC}_{50}$ values of 2 against recombinant Src V284C, JNK2 V40C/M108T, EphA2 V627C and PAK1 V286C/M346T, and WT Src, JNK2, EphA2 and PAK1. Values shown are mean \pm SEM, $n=3$. (B) $\mathrm{IC}_{50}$ values of 3 against recombinant Src V284C, JNK2 V40C/M108T, EphA2 V627C and PAK1 V286C/M346T, and wild-type Src, JNK2, EphA2 and PAK1. Values shown are mean \pm SEM, $n=3$. (C) 2-TCO and 3-TCO selectively enrich drug-sensitized JNK2 (V40C/M108T) from 
cell lysates. HEK293 cell lysate containing either JNK2 (V40C/M108T) or JNK2 WT were treated with 2-TCO $(10 \mu \mathrm{M})$ or 3-TCO $(10 \mu \mathrm{M})$, incubated with Tz-beads, and captured proteins were eluted under reducing and denaturing conditions. An anti-JNK2 immunoblot of the flow through (FT) and elution (EL) are shown. (D) 2-TCO selectively enriches drug-sensitized BTK (V416C) from cells. HEK293 cells transiently expressing either N-terminal Flag-tagged BTK (V416C) or BTK WT were treated with 2-TCO $(2.5 \mu \mathrm{M})$, lysed, and then incubated with Tz-beads. Captured proteins were eluted under reducing and denaturing conditions. An anti-DYKDDDDK Tag (Flag Tag) immunoblot of the input (IN) and elution (EL) are shown. Note: in Figure S4D, elution samples were 2-fold more concentrated than input samples. 
A

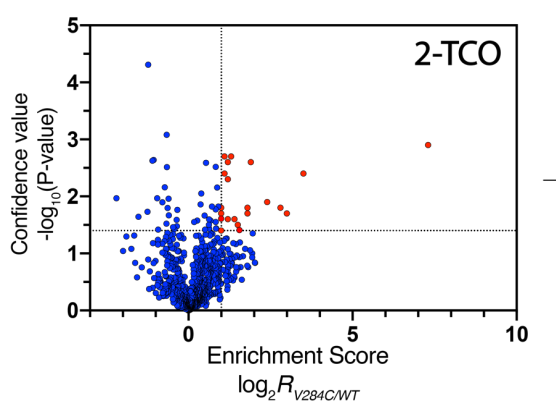

B

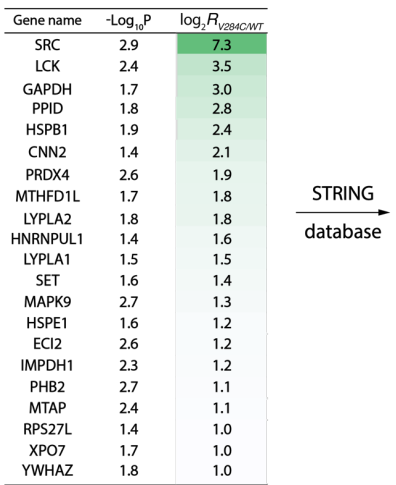

C

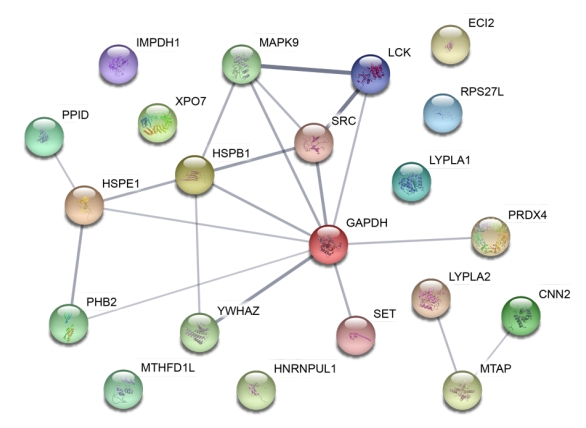

Figure S5. Interrogation of growth-factor-stimulated 2-TCO-bound Src's interactome with coclickable precipitation. (A) Volcano plot showing the $\log _{2}$ transformed MaxQuant intensities of the label-free comparison of 2-TCO-treated, growth factor-stimulated HeLa cells expressing Src V284C or Src WT $(n=3)$. Statistically significant interactors for drug-sensitized Src were defined as protein hits with enrichment scores $=\log _{2}$ [Intensity (drug-sensitized target)/Intensity (WT target $)] \geq 1.0$ and confidence values $=-\log _{10}(\mathrm{P}$-value $) \geq 1.4$. The calculation was based on intensity values computed by MaxQuant. Missing protein intensity values were imputed by Perseus with a distribution downshift of 1.3 and a width of 0.2. Specific Src interactors are highlighted in red and non-specific binders are marked in blue. (B) Proteins that show statistically significant enrichment with 2-TCO from Src V284C-expressing, growth factor-stimulated HeLa cells. The second column shows confidence values $\left(-\log _{10}(\mathrm{P}\right.$-value $\left.)\right)$ and the third column shows enrichment scores. (C) STRING network of the interactome of 2-TCO-bound Src V284C. Significant interactors were visualized using the STRING database., ${ }^{2}$ Each circle (node) represents a member of the interactome. Each line between the nodes (edge) represents a detected protein-protein interaction (PPI) according to the STRING database. 
A

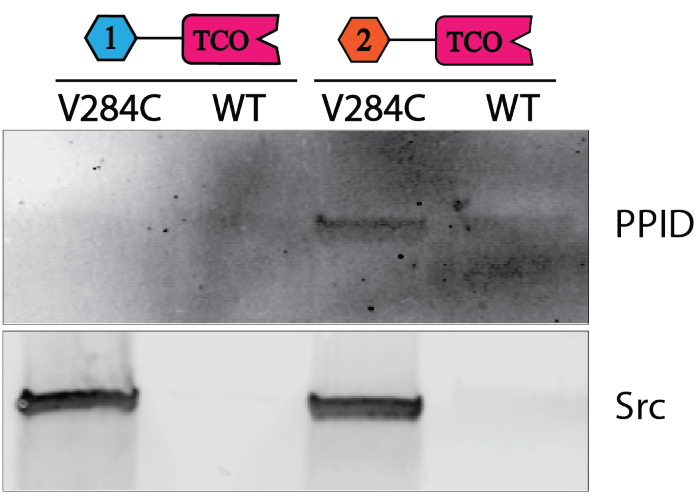

B

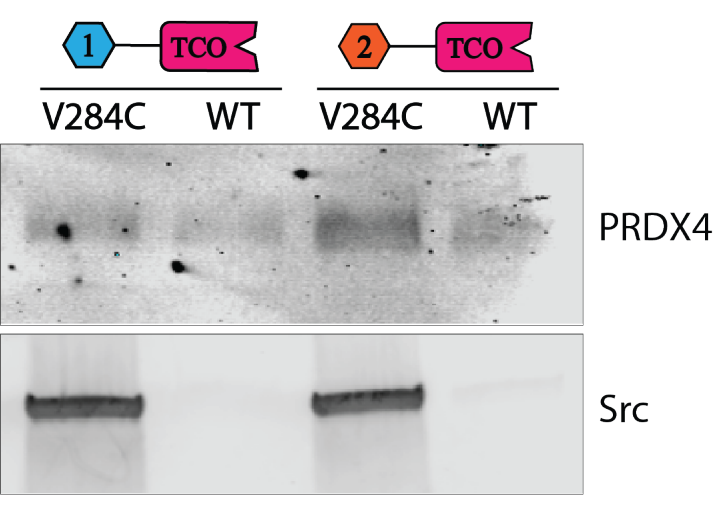

Figure S6. Interrogation of Src's interactome with co-clickable precipitation. (A) Western blot confirmation of PPID as an interactor with the 2-TCO-bound Src V284C complex. An anti-PPID immunoblot of the elution (EL) is shown (top). An anti- Src immunoblot of the elution (EL) is also shown (bottom). (B) Western blot confirmation of PRDX4 as an interactor with the 2-TCO-bound Src V284C complex. An anti-PRDX4 immunoblot of the elution (EL) is shown (top). An anti-Src immunoblot of the elution (EL) is also shown (bottom). 


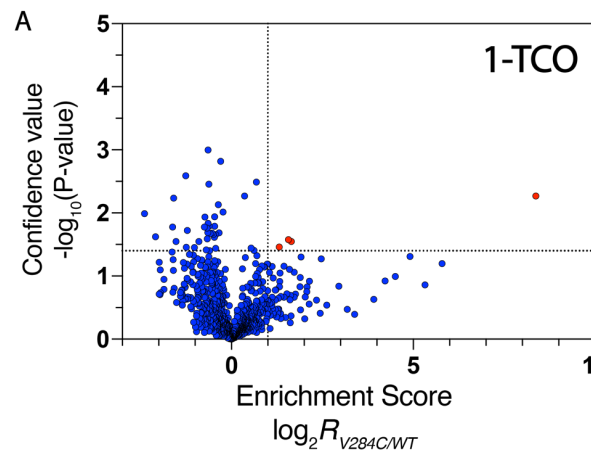

B

C
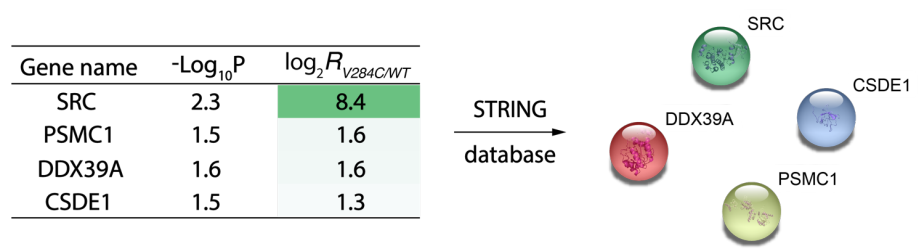

D

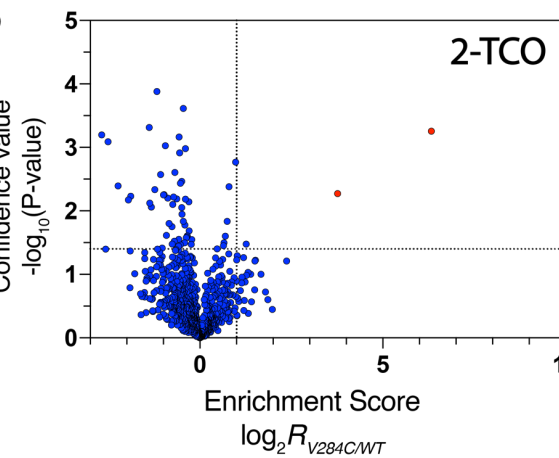

$\rightarrow$\begin{tabular}{ccccc}
\hline Gene name & $-\log _{10} \mathrm{P}$ & $\log _{2} R_{\text {V284CNT }}$ & & \multicolumn{1}{c}{ STRING } \\
\hline SRC & 3.3 & 6.3 & & database \\
GOLGA4 & 2.3 & 3.8 & &
\end{tabular}

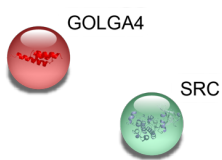

Figure S7. Characterization of 1-TCO-bound and 2-TCO-bound Src's interactome in serumstarved HeLa cells. (A, D) Volcano plot showing the $\log _{2}$ transformed MaxQuant intensities of the label-free comparison of 1-TCO-treated (A, top) or 2-TCO-treated (D, bottom), serum-starved HeLa cells expressing Src V284C or Src WT ( $n=3)$. Statistically significant interactors for drugsensitized Src were defined as protein hits with enrichment scores $=\log _{2}$ [Intensity (drug-sensitized target)/Intensity (WT target) $] \geq 1.0$ and confidence values $=-\log _{10}(\mathrm{P}$-value $) \geq 1.4$. The calculation was based on intensity values computed by MaxQuant. Missing protein intensity values were imputed by Perseus with a distribution downshift of 1.3 and a width of 0.2 . Specific Src interactors are highlighted in red and non-specific binders are marked in blue. (B, E) Proteins that significantly co-enriched with 1-TCO (B, top) or 2-TCO (E, bottom) from Src V284C-expressing, serumstarved HeLa cells. The second column shows confidence values (- $\log _{10}(\mathrm{P}$-value) $)$ and the third column shows enrichment scores. (C, F) STRING network of the interactome of 1-TCO-bound (C, top) or 2-TCO-bound (F, bottom) Src V284C. Significant interactors were visualized using the STRING database. Each circle (node) represents a member of the interactome. 
A

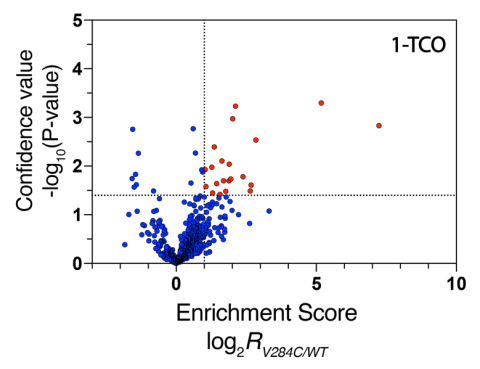

D

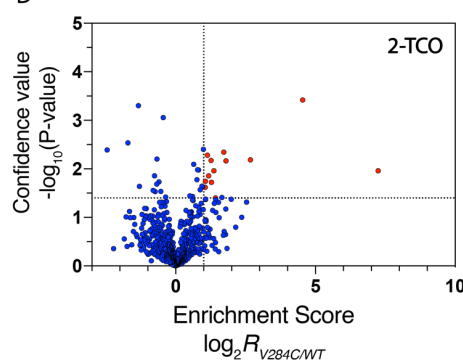

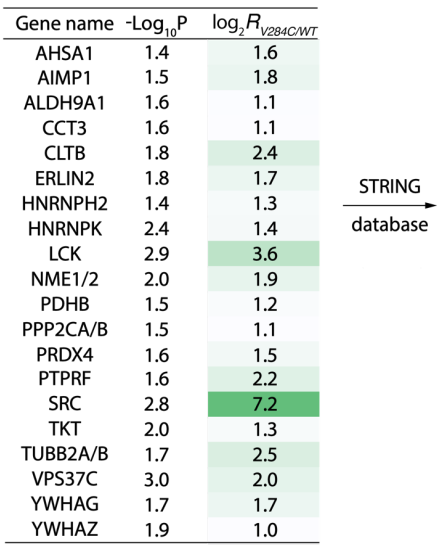

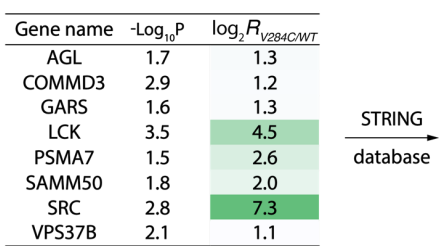

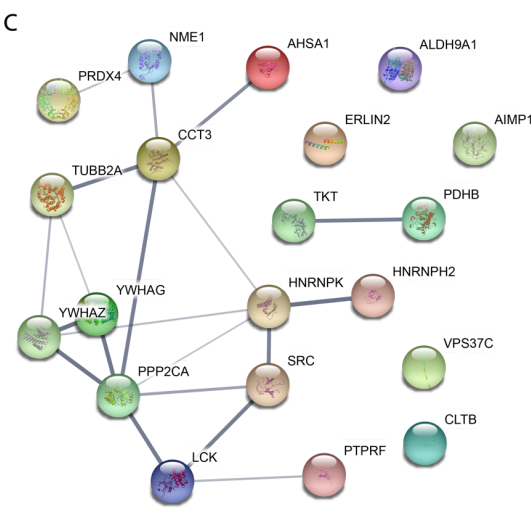

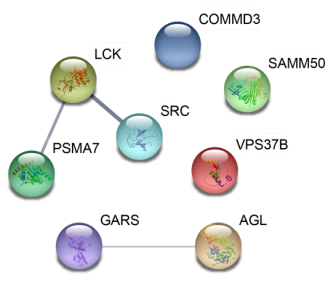

Figure S8. Characterization of 1-TCO-bound and 2-TCO-bound Src's interactome in Hela cells cultured in complete medium (DMEM). (A, D) Volcano plot showing the $\log _{2}$ transformed MaxQuant intensities of the label-free comparison of 1-TCO-treated (A, top) or 2-TCO-treated (D, bottom) HeLa cells expressing Src V284C or Src WT ( $n=3)$ cultured in complete medium (DMEM). Statistically significant interactors for drug-sensitized Src were defined as protein hits with enrichment scores $=\log _{2}$ [Intensity (drug-sensitized target)/Intensity (WT target) $] \geq 1.0$ and confidence values $=-\log _{10}(\mathrm{P}$-value $) \geq 1.4$. The calculation was based on intensity values computed by MaxQuant. Missing protein intensity values were imputed by Perseus with a distribution downshift of 1.3 and a width of 0.2. Specific Src interactors are highlighted in red and non-specific binders are marked in blue. (B, E) Proteins that significantly co-enriched with 1-TCO (B, top) or 2-TCO (E, bottom) from Src V284C-expressing HeLa cells cultured in complete medium (DMEM). The second column shows confidence values $\left(-\log _{10}(\mathrm{P}\right.$-value $\left.)\right)$ and the third column shows enrichment scores. (C, F) STRING network of the interactome of 1-TCO-bound (C, top) or 2-TCO-bound (F, bottom) Src V284C. Significant interactors were visualized using the STRING database. Each circle (node) represents a member of the interactome. Each line between the nodes (edge) represents a detected protein-protein interaction (PPI) according to the STRING database. 
A

B

\begin{tabular}{|c|c|c|c|c|c|c|c|c|}
\hline Gene name & $-\log _{10} P$ & $\log _{2} R_{V 284 C W T}$ & Gene name & $-\log _{10} P$ & $\log _{2} R_{V 284 C W T}$ & Gene name & $-\log _{10} P$ & $\log _{2} R_{V 284 C N T}$ \\
\hline ACACA & 1.5 & 1.3 & LGALS1 & 2.6 & 5.4 & SEC16A & 1.7 & 2.3 \\
\hline ACAT2 & 1.8 & 1.6 & MAPK9 & 2.0 & 1.5 & SEC23A & 1.5 & 1.9 \\
\hline AIMP1 & 2.4 & 2.6 & MARS & 3.2 & 2.6 & SEH1L & 1.6 & 2.6 \\
\hline AIMP2 & 4.5 & 5.9 & MAT2A & 1.8 & 2.4 & SFXN1 & 2.4 & 1.6 \\
\hline ALDH1B1 & 1.5 & 3.4 & MDN1 & 2.2 & 1.2 & SLC25A10 & 1.5 & 4.0 \\
\hline ATP5C1 & 1.4 & 4.6 & NDUFA10 & 3.8 & 5.2 & SMN1 & 2.5 & 2.3 \\
\hline CAD & 1.9 & 2.5 & PDHB & 1.8 & 2.3 & SRC & 2.5 & 6.6 \\
\hline CBL & 3.9 & 5.3 & PPP2R2A & 1.8 & 2.0 & SUB1 & 3.3 & 3.9 \\
\hline CBR1 & 3.1 & 5.8 & PRDX2 & 1.5 & 1.7 & TCP1 & 1.5 & 2.8 \\
\hline CCT4 & 1.5 & 1.4 & PRMT5 & 1.5 & 2.3 & TKT & 2.2 & 1.5 \\
\hline CCT7 & 1.5 & 1.9 & PSMA5 & 3.3 & 4.6 & TSG101 & 1.9 & 3.0 \\
\hline CNOT1 & 2.0 & 2.6 & $\mathrm{RCC} 2$ & 1.7 & 1.5 & TUBA1B & 1.5 & 1.5 \\
\hline COPB2 & 1.7 & 1.5 & RPL10A & 1.8 & 1.5 & UBE2C & 1.5 & 1.1 \\
\hline DARS & 1.8 & 2.8 & RPL19 & 2.5 & 3.4 & UBE2S & 2.9 & 4.3 \\
\hline DCXR & 3.3 & 3.0 & RPL27A & 2.1 & 3.2 & VAPA & 3.1 & 1.0 \\
\hline DNAJA1 & 1.7 & 2.5 & RPL4 & 1.4 & 4.1 & VDAC3 & 1.8 & 1.6 \\
\hline ECM29 & 2.4 & 1.1 & RPL7 & 1.9 & 4.4 & YES1 & 4.9 & 8.7 \\
\hline HIST1H1 & 2.1 & 3.4 & RPLP1 & 2.3 & 1.2 & YWHAB & 1.7 & 1.5 \\
\hline HNRNPK & 1.8 & 1.6 & RPL14 & 1.8 & 3.9 & YWHAG & 1.6 & 2.9 \\
\hline HSD17B10 & 2.2 & 1.3 & RPS15A & 2.6 & 3.6 & ZBTB1 & 2.0 & 3.3 \\
\hline
\end{tabular}
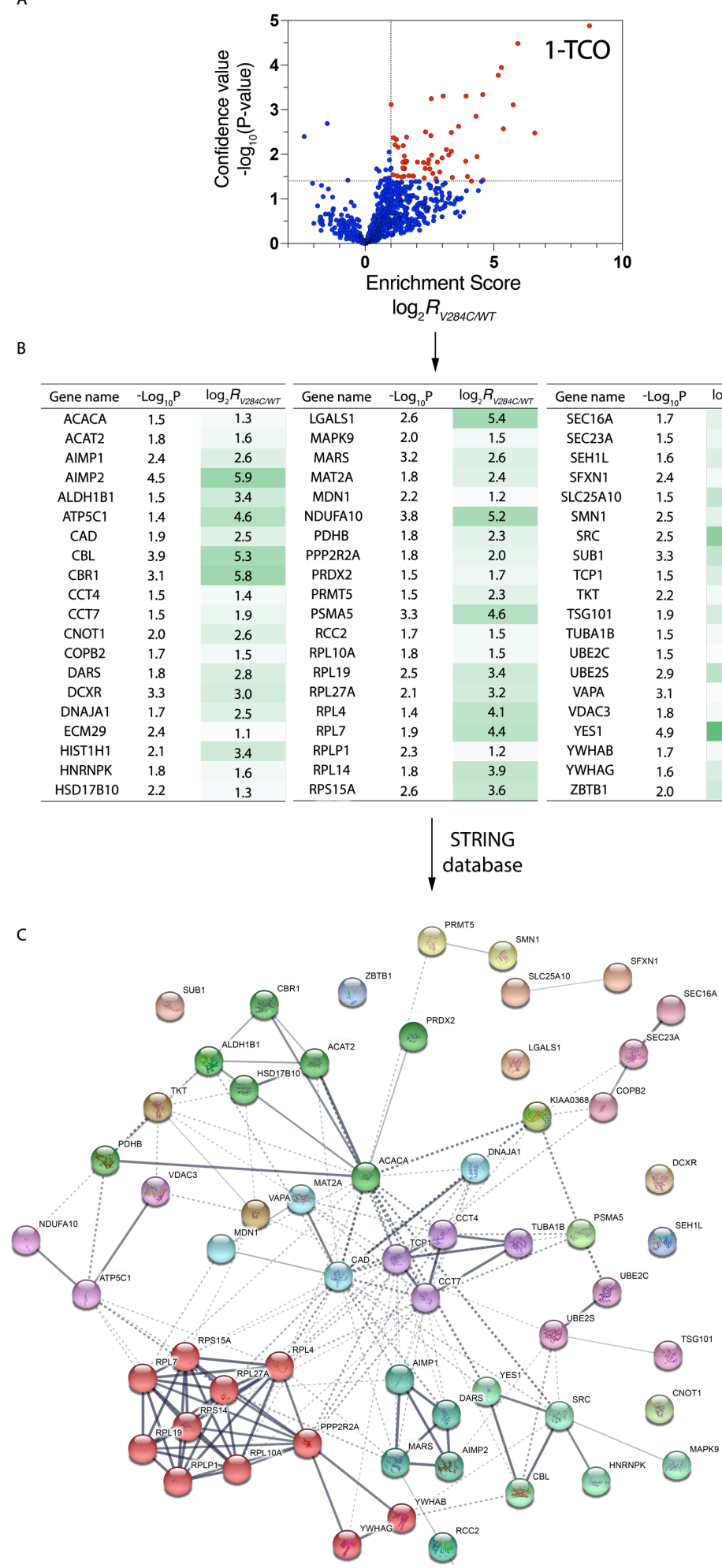

D
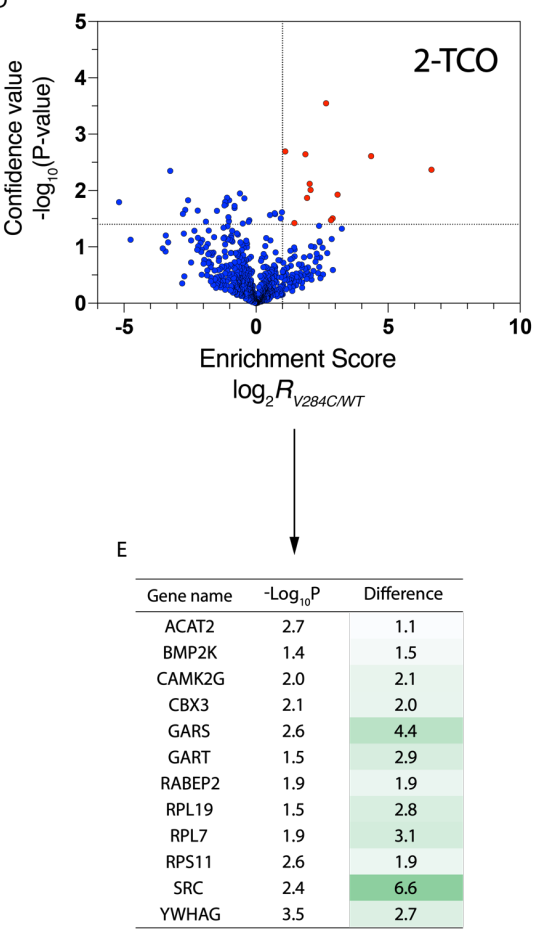

STRING

database
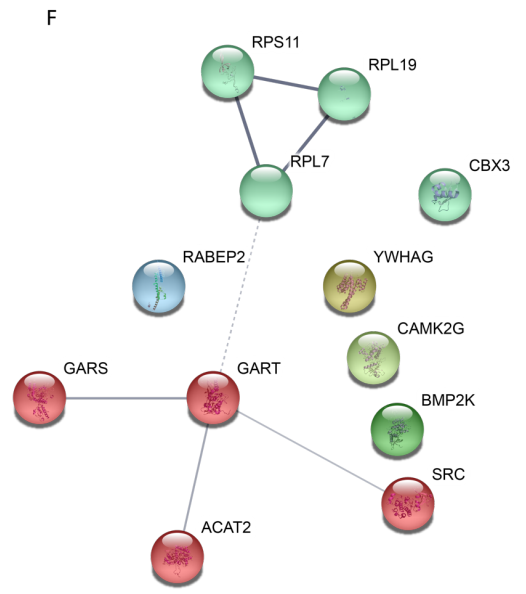

Figure S9. Characterization of 1-TCO-bound and 2-TCO-bound Src's interactome in HeLa cells plated on fibronectin. (A, D) Volcano plot showing the $\log _{2}$ transformed MaxQuant intensities of the label-free comparison of 1-TCO-treated (A, left) or 2-TCO-treated (D, right) HeLa cells expressing Src V284C or Src WT $(n=3)$ plated on fibronectin. Statistically significant interactors for drug-sensitized $\mathrm{Src}$ were defined as protein hits with enrichment scores $=\log _{2}$ [Intensity (drug- 
sensitized target)/Intensity $($ WT target $)] \geq 1.0$ and confidence values $=-\log _{10}(\mathrm{P}$-value $) \geq 1.4$. The calculation was based on intensity values computed by MaxQuant. Missing protein intensity values were imputed by Perseus with a distribution downshift of 1.3 and a width of 0.2. Specific Src interactors are highlighted in red and non-specific binders are marked in blue. (B, E) Proteins that significantly co-enriched with 1-TCO (B, left) or 2-TCO (E, right) from Src V284C-expressing HeLa cells plated on fibronectin. The second column shows confidence values $\left(-\log _{10}(\mathrm{P}-\mathrm{value})\right)$ and the third column shows enrichment scores. (C, F) Significant interactors were visualized using the STRING database. Each circle (node) represents a member of the interactome. Each line between the nodes (edge) represents a detected protein-protein interaction (PPI) according to the STRING database. 


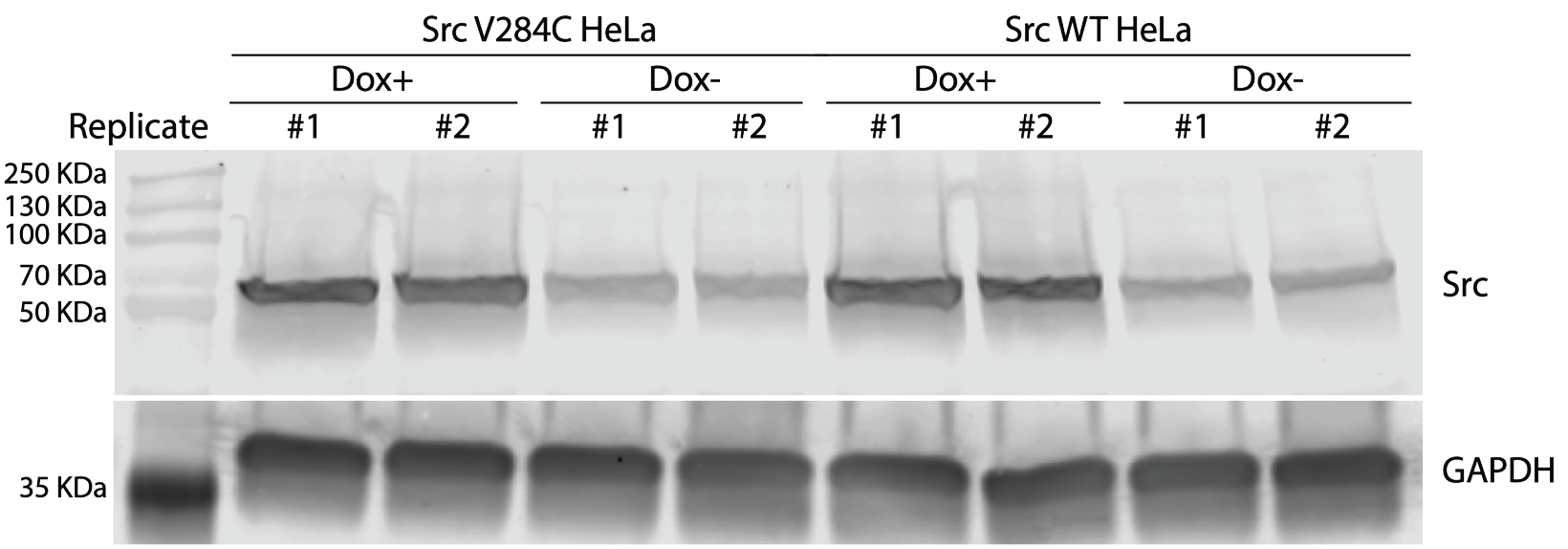

Figure S10. Anti-Src immunoblots of HeLa cells stably expressing Src V284C or Src WT ( $n=2)$. Src V284C and Src WT expression was induced with $1 \mu \mathrm{g} / \mathrm{mL}$ of doxycycline for 24 hours prior to performing PLA studies. 


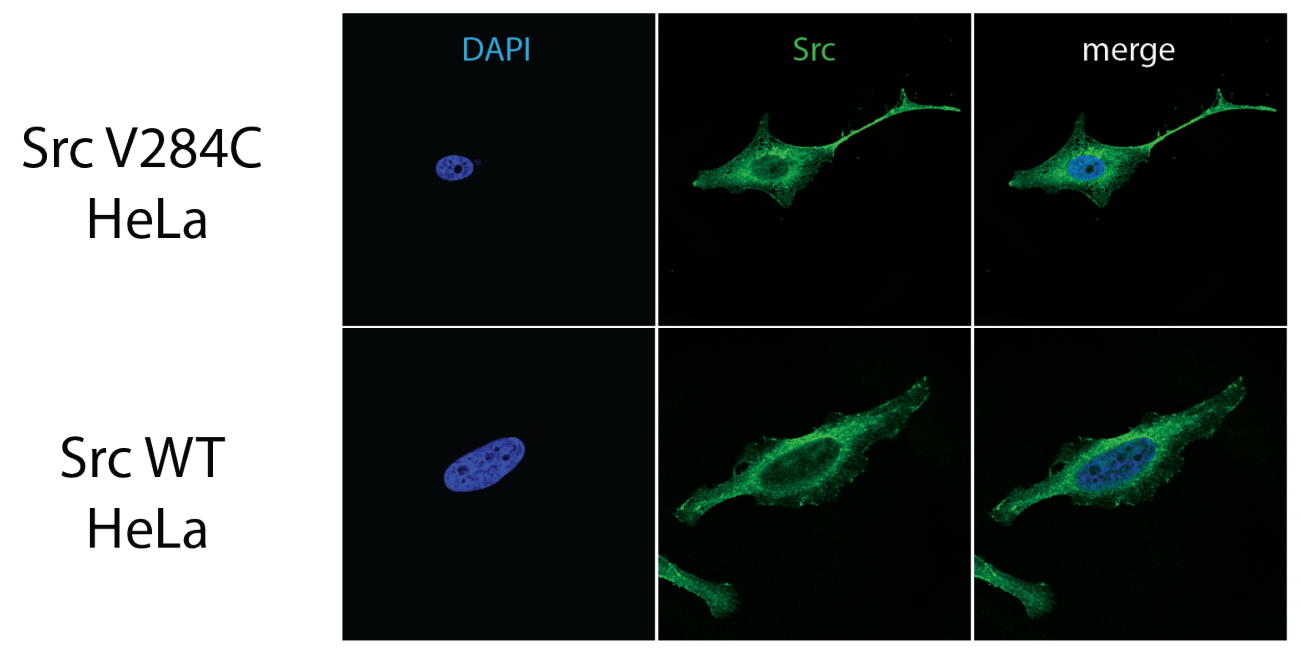

B

Anti-Src mAb: - (negative control)

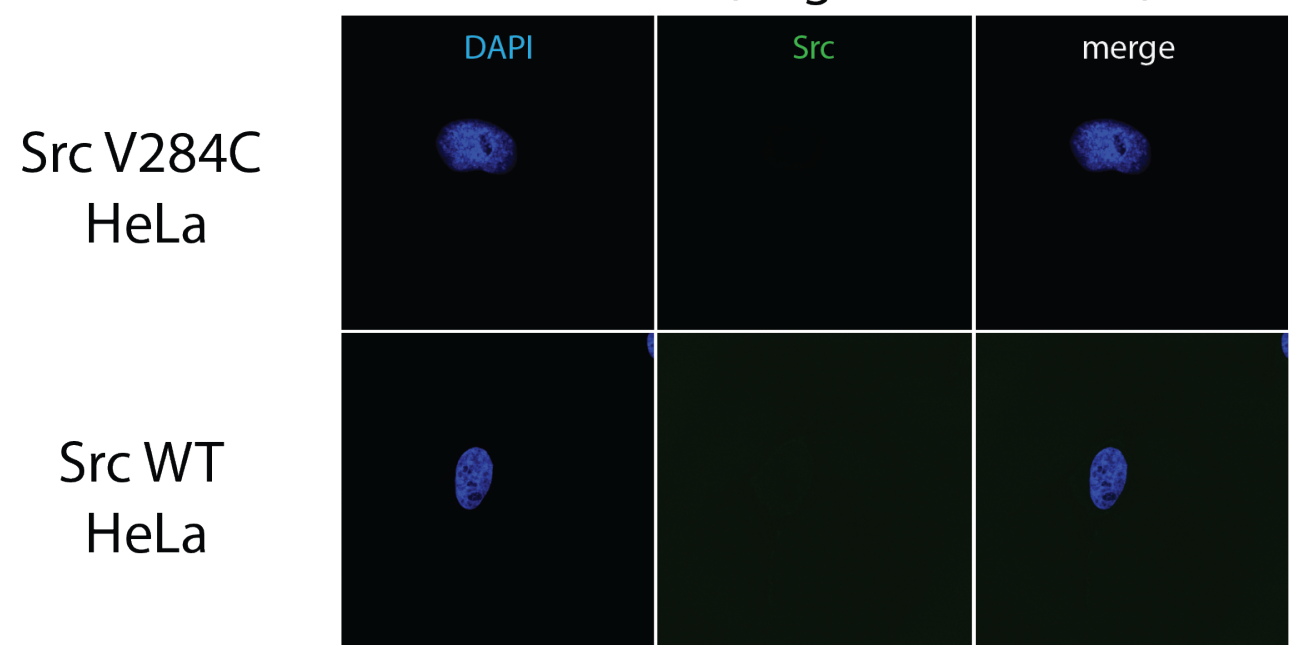

Figure S11. Cellular localization of Src V284C and Src WT in HeLa cells prior to treatment with TCO probes. (A) Immunofluorescent analysis of Src V284C (top) and Src WT (bottom) in HeLa cells. HeLa cells stably expressing Src V284C (top) or Src WT (bottom) were fixed and incubated with an anti-Src antibody, followed by a Mega 485-conjugated secondary antibody. The nucleus was visualized using DAPI. (B) Negative control of immunofluorescent analysis. HeLa cells stably expressing Src V284C (top) or Src WT (bottom) were fixed and incubated with only a Mega 485conjugated secondary antibody. The nucleus was visualized using DAPI. 


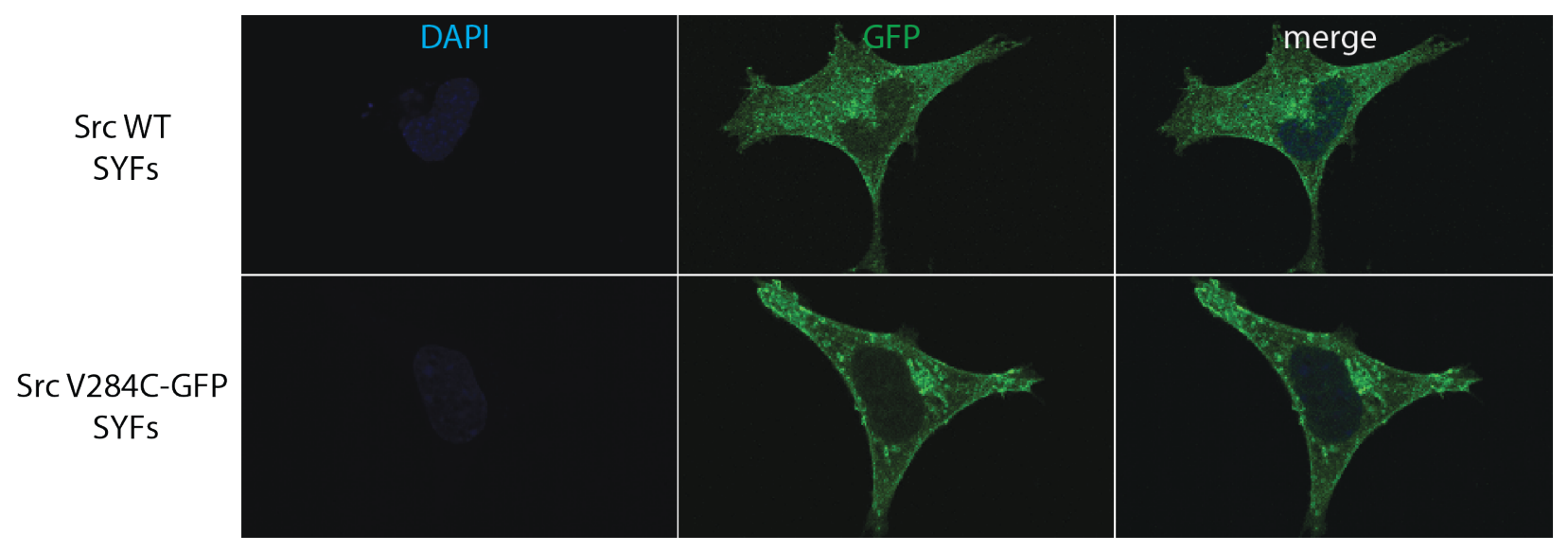

Figure S12. Cellular localization of C-terminal GFP-tagged full-length Src WT (top) and Src V284C (bottom) in Src/Yes/Fyn (-/-/-) fibroblast cells (SYFs). SYFs cells that transiently express Src WT-GFP (top) or Src V284C-GFP (bottom) were fixed with 4\% para-formaldehyde. The nucleus was visualized using DAPI. GFP-tagged Src constructs were visualized under GFP channel (excitation: $488 \mathrm{~nm}$; emission: $510 \mathrm{~nm}$ ). 


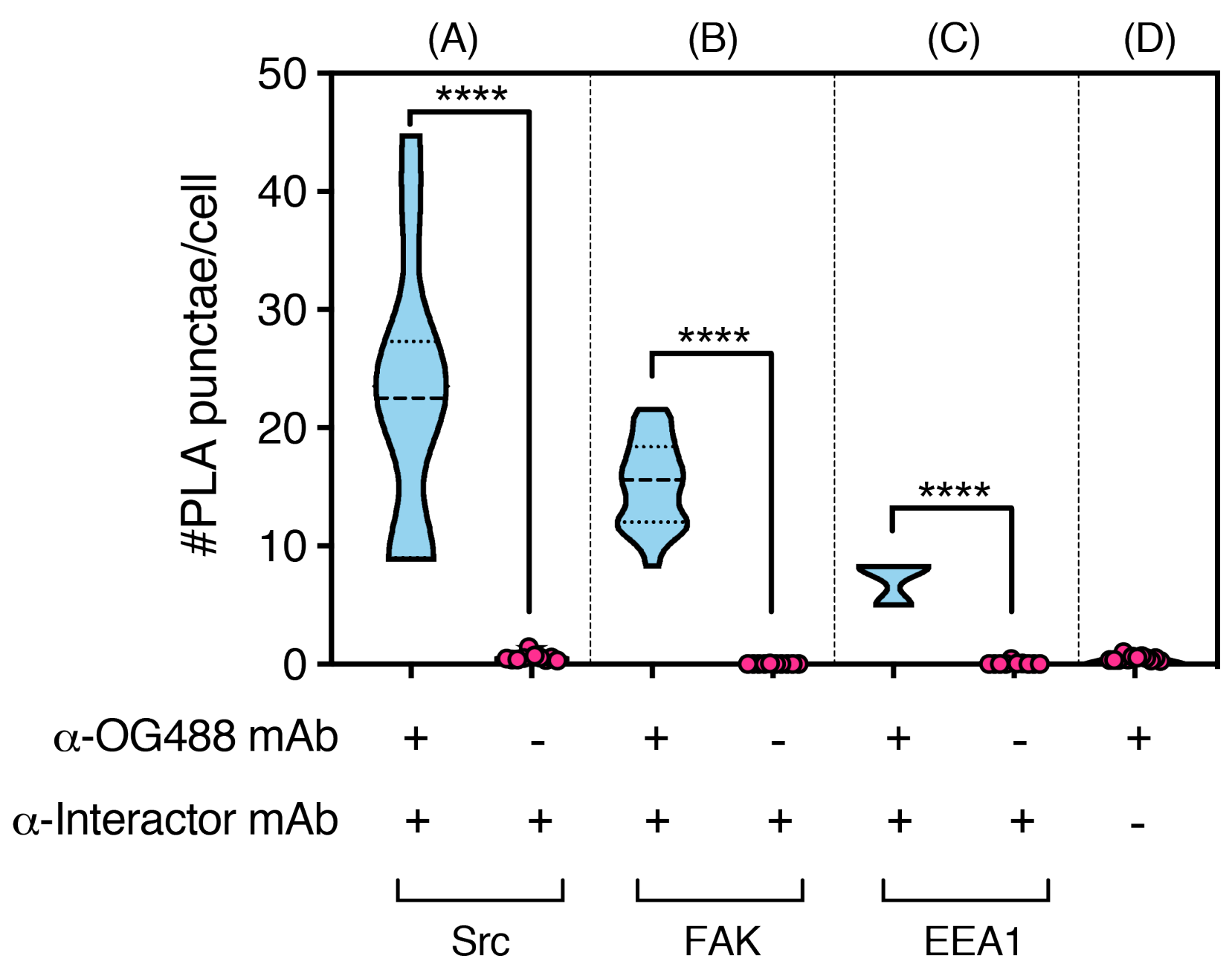

Figure S13. Technical negative controls of proximity ligation assays (PLAs) in HeLa cells treated by 1-TCO. (A) Technical negative control of PLA for visualization of the probe-bound target. Quantified signals for the number of PLA-mediated fluorescent puncta observed per cell are shown in the violin plot. Values shown are the means of fluorescent puncta per cell from at least three independent experiments (number of total cells quantified for control experiments: $n=308$ ). (B) Technical negative control of the Src/FAK interaction PLA. Quantified signals for the number of PLA-mediated fluorescent puncta observed per cell are shown in the violin plot. Values shown are the means of fluorescent puncta per cell from at least three independent experiments. (number of total cells quantified for control experiments: $n=464)$. (C) Technical negative control of the cellular organelle localization PLA. Quantified signals for the number of PLA-mediated fluorescent puncta observed per cell are shown in the violin plot. Values shown are the means of fluorescent puncta per cell from at least three independent experiments. (number of total cells quantified for control experiments: $n=209$ ). (D) Technical negative control of PLA with the anti-Oregon Green488 antibody. Quantified signals for the number of PLA-mediated fluorescent puncta observed per cell are shown in the violin plot. Values shown are the means of fluorescent puncta per cell from at least three independent experiments. (number of total cells quantified for control experiments: $n=263$ ). 


\section{Materials and Reagents}

Lysis Buffer and Washing Buffer 1: [Tris $]=50 \mathrm{mM}, \mathrm{pH}=7.8 ;[\mathrm{NaCl}]=120 \mathrm{mM} ;[\mathrm{NaF}]=10 \mathrm{mM}$;

$\left[\mathrm{Na}_{3} \mathrm{VO}_{4}\right]=1 \mathrm{mM} ;[$ EDTA $]=1 \mathrm{mM}$; [IGEPAL CA-630]= 1\% (by volume); Protease Inhibitor Tablet (Pierce)

Washing Buffer 2: $[$ Tris $]=50 \mathrm{mM}, \mathrm{pH}=7.8 ;[\mathrm{NaCl}]=120 \mathrm{mM}$; [EDTA $]=1 \mathrm{mM}$.

Denaturing Buffer: [Tris] $=50 \mathrm{mM}, \mathrm{pH}=8.5$; [Guanidinium Chloride] $=6 \mathrm{M}$; [TCEP] $=5 \mathrm{mM}$ (add freshly); [CAM]= $10 \mathrm{mM}$ (add freshly).

Pierce Protease Inhibitor Tablets (Thermo Scientific; Catalog number: A32963)

NHS-Activated Sepharose 4 Fast Flow (GE Healthcare; Catalog number: 17090601)

Pierce 660 nm Protein Assay (Thermo Scientific)

Lys-c Lysyl Endopeptidase, MS Grade (Wako)

MS Grade Trypsin (Pierce)

Grace Bio-labs CultureWell chambered coverglass (8 wells, well diam. $\times$ thickness $6 \mathrm{~mm} \times 1 \mathrm{~mm}$, well volume 15-30 $\mu \mathrm{L}$; Sigma-Aldrich, cat. no. GBL103380)

\section{Antibodies:}

Anti-Src antibody (Cell Signaling Technology, cat. no. \#2109)

Anti-FAK antibody (Cell Signaling Technology, cat. no. \#71433)

Anti-c-Cbl antibody (Cell Signaling Technology, cat. no. \#2747)

Anti-EGFR antibody (Cell Signaling Technology, cat. no. \#4267)

Anti-EEA1 antibody (Cell Signaling Technology, cat. no. \#3288)

Anti-Oregon Green488 antibody (Thermo Fisher, cat. no. A-11095)

Anti-His Tag antibody (Abcam, cat. no. G020) 
Anti-DYKDDDDK Tag antibody (Cell Signaling Technology, cat. no. \#2368)

Anti-SAPK/JNK antibody (Cell Signaling Technology, cat. no. \#9252)

Anti-PPID antibody (ABclonal, cat. no. \#A6949)

Anti-PRDX4 antibody (ABclonal, cat.no. \#A1486)

Anti-GAPDH antibody (Abcam, cat. no. 9485)

Duolink in situ mounting medium with DAPI (Sigma-Aldrich, cat. no. DUO82040)

Duolink in situ PLA probe anti-rabbit PLUS (Sigma-Aldrich, cat. no. DUO92002)

Duolink in situ PLA probe anti-goat MINUS (Sigma-Aldrich, cat. no. DUO92006)

Duolink in situ detection reagents orange (Sigma-Aldrich, cat. no. DUO92007)

Duolink in situ wash buffers, Fluorescence (Sigma-Aldrich, cat. no. DUO82049) 


\section{Generation of Stable HeLa Cell Line}

Doxycycline-inducible HeLa cells expressing Src V284C or Src WT were generated using Flp-In T-REx HeLa cells (a generous gift from Stephen S. Taylor, University of Manchester). Flp-In TREx HeLa cells were co-transfected with Src V284C-pcDNA5/FRT/TO or Src WTpcDNA5/FRT/TO vector, and pOG44 Flp recombinase vector (Thermo) (1:2= pcDNA5/FRT/TO:pOG) using Lipofectamine (1 $\mu \mathrm{g}$ of DNA: $3 \mu \mathrm{L}$ of Lipofectamine). Stably transfected HeLa cells were selected with $200 \mu \mathrm{g} / \mathrm{mL}$ of hygromycin for 7-10 d. After induction with $1 \mu \mathrm{g} / \mathrm{mL}$ of doxycycline, expression of the desired transgenes was verified using Western blot analysis.

\section{Inserted gene of Src V284C:}

MGSNKSKPKDASQRRRSLEPAENVHGAGGGAFPASQTPSKPASADGHRGPSAAFAPAA AEPKLFGGFNSSDTVTSPQRAGPLAGGVTTFVALYDYESRTETDLSFKKGERLQIVNNTE GDWWLAHSLSTGQTGYIPSNYVAPSDSIQAEEWYFGKITRRESERLLLNAENPRGTFLV RESETTKGAYCLSVSDFDNAKGLNVKHYKIRKLDSGGFYITSRTQFNSLQQLVAYYSKH ADGLCHRLTTVCPTSKPQTQGLAKDAWEIPRESLRLEVKLGQGCFGECWMGTWNGTTR VAIKTLKPGTMSPEAFLQEAQVMKKLRHEKLVQLYAVVSEEPIYIVTEYMSKGSLLDFL KGETGKYLRLPQLVDMAAQIASGMAYVERMNYVHRDLRAANILVGENLVCKVADFGL ARLIEDNEYTARQGAKFPIKWTAPEAALYGRFTIKSDVWSFGILLTELTTKGRVPYPGMV NREVLDQVERGYRMPCPPECPESLHDLMCQCWRKEPEERPTFEYLQAFLEDYFTSTEPQ YQPGENL* 
Inserted gene of Src WT:

MGSNKSKPKDASQRRRSLEPAENVHGAGGGAFPASQTPSKPASADGHRGPSAAFAPAA AEPKLFGGFNSSDTVTSPQRAGPLAGGVTTFVALYDYESRTETDLSFKKGERLQIVNNTE GDWWLAHSLSTGQTGYIPSNYVAPSDSIQAEEWYFGKITRRESERLLLNAENPRGTFLV RESETTKGAYCLSVSDFDNAKGLNVKHYKIRKLDSGGFYITSRTQFNSLQQLVAYYSKH ADGLCHRLTTVCPTSKPQTQGLAKDAWEIPRESLRLEVKLGQGCFGEVWMGTWNGTT RVAIKTLKPGTMSPEAFLQEAQVMKKLRHEKLVQLYAVVSEEPIYIVTEYMSKGSLLDF LKGETGKYLRLPQLVDMAAQIASGMAYVERMNYVHRDLRAANILVGENLVCKVADFG LARLIEDNEYTARQGAKFPIKWTAPEAALYGRFTIKSDVWSFGILLTELTTKGRVPYPGM VNREVLDQVERGYRMPCPPECPESLHDLMCQCWRKEPEERPTFEYLQAFLEDYFTSTEP QYQPGENL* 


\section{Synthesis of TCO probes (1-TCO, 2-TCO, and 3-TCO)}

All chemicals purchased from commercial suppliers were used without further purification unless otherwise stated. Reactions were monitored with thin-layer chromatography (TLC) using silica gel 60 F254 coated glass plates (EM Sciences). Compound purification was performed with an IntelliFlash 280 automated flash chromatography system using pre-packed Varian SuperFlash silica gel columns (Hexane/EtOAc or $\mathrm{CH}_{2} \mathrm{Cl}_{2} / \mathrm{MeOH}$ gradient solvent). A Varian Dynamax Microsorb 100-5 C18 column (250 mm x $21.4 \mathrm{~mm}$ ), eluting with $\mathrm{H}_{2} \mathrm{O} / \mathrm{CH}_{3} \mathrm{CN}$ or $\mathrm{H}_{2} \mathrm{O} / \mathrm{MeOH}$ gradient solvent $(+0.05 \%$ TFA), was used for preparatory HPLC purification. The purity of all final compounds was determined by analytical HPLC with an Agilent ZORBAX SB-C18 $(2.1 \mathrm{~mm}$ x $150 \mathrm{~mm}$ ) or Varian Microsorb-MV 100-5 C18 column (4.6 mm x $150 \mathrm{~mm})$, eluting with either $\mathrm{H}_{2} \mathrm{O} / \mathrm{CH}_{3} \mathrm{CN}$ or $\mathrm{H}_{2} \mathrm{O} / \mathrm{MeOH}$ gradient solvent ( $+0.05 \%$ TFA). Elution was monitored by a UV detector at $\lambda=220 \mathrm{~nm}$ and $\lambda=254 \mathrm{~nm}$, with all final compounds displaying $>95 \%$ purity. Nuclear

magnetic resonance (NMR) spectra were recorded on Bruker 300 or $500 \mathrm{MHz}$ NMR spectrometers at ambient temperature. Chemical shifts were reported in parts per million (ppm) and coupling constants in hertz (Hz). ${ }^{1} \mathrm{H}-\mathrm{NMR}$ spectra were referenced to the residual solvent peaks as internal standards (7.26 ppm for $\mathrm{CDCl}_{3}, 2.50 \mathrm{ppm}$ for d6-DMSO, and $3.34 \mathrm{ppm}$ for $\mathrm{CD}_{3} \mathrm{OD}$ ). Mass spectra were recorded with a Bruker Esquire Liquid Chromatograph - Ion Trap Mass Spectrometer. 


\section{Synthesis of 1-TCO}
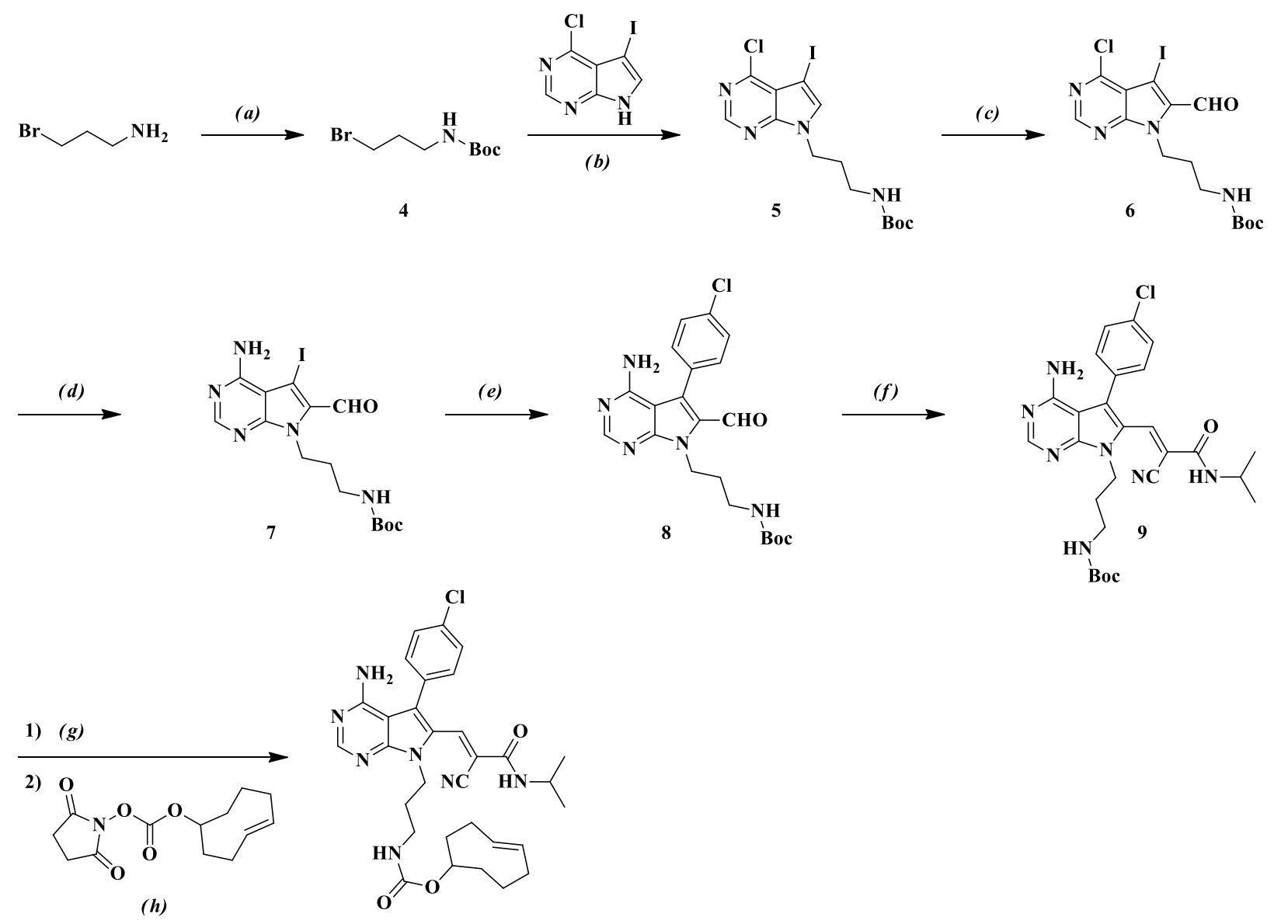

1-TCO

Reaction scheme for 1-TCO: (a) $\mathrm{Boc}_{2} \mathrm{O}, \mathrm{Et}_{3} \mathrm{~N}, \mathrm{DCM}, 0{ }^{\circ} \mathrm{C}-\mathrm{rt}, 95 \%$; (b) $\mathrm{Cs}_{2} \mathrm{CO}_{3}, \mathrm{DMF}, 50{ }^{\circ} \mathrm{C}, 14$ hours, 59\%; (c) Diisopropylamine, $n$-BuLi, ethyl formate, THF, $-78{ }^{\circ} \mathrm{C}$-rt, overnight, $22 \%$; (d) Ammonium hydroxide, 1,4-Dioxane, $40{ }^{\circ} \mathrm{C}, 3$ hours, 43\%; (e) 4-Chlorophenylboronic acid, $\mathrm{K}_{3} \mathrm{PO}_{4}, \mathrm{Pd}(\mathrm{dppf})_{2} \mathrm{Cl}_{2}, 1$,4-Dioxane, $\mathrm{H}_{2} \mathrm{O}, 90{ }^{\circ} \mathrm{C}, 130$ mins (microwave), 74\%; (f) 2-Cyano- $N$ isopropylacetamide, DBU, THF, rt, 6 hours, 50\%; (g) TFA, DCM, rt, 30 mins; (h) DIPEA, THF, rt, overnight, $60 \%$. 
Tert-butyl (3-bromopropyl)carbamate (4)

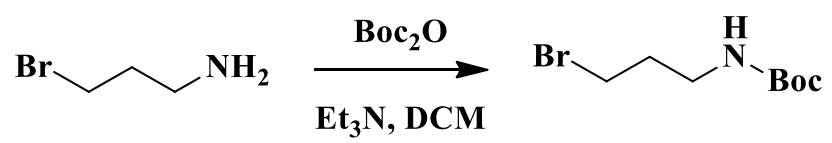

3-Bromopropylamine hydrobromide (5.3 g, $24 \mathrm{mmol}, 1.0$ equiv.) and triethylamine (2.7 $\mathrm{g}, 26$ mmol, $3.6 \mathrm{~mL}, 1.1$ equiv.) were dissolved in anhydrous dichloromethane (50 mL) and cooled to $0^{\circ} \mathrm{C}$ in a $250-\mathrm{mL}$ round bottom flask under nitrogen. A solution of di-tert-butyl dicarbonate $(5.3 \mathrm{~g}$, $24 \mathrm{mmol}, 1.0$ equiv. $)$ in dichloromethane $(40 \mathrm{~mL})$ was added dropwise to the above solution over 30 minutes. The resulting reaction mixture was allowed to warm to room temperature overnight. The reaction was then quenched with aqueous sodium hydroxide solution ( $20 \mathrm{~mL}, 5 \%$ by weight). The organic phase was washed with water $(20 \mathrm{~mL})$, brine $(20 \mathrm{~mL})$ and then dried over anhydrous sodium sulfate. The solvent was removed in vacuo and afforded tert-butyl (3bromopropyl)carbamate as a white solid $(5.4 \mathrm{~g}, 95 \%)$. The crude product was used in further steps without purification. ${ }^{1} \mathrm{H}-\mathrm{NMR}\left(300 \mathrm{MHz}, \mathrm{CDCl}_{3}\right) \delta=4.64(\mathrm{~s}, 1 \mathrm{H}), 3.44(\mathrm{t}, J=6.5 \mathrm{~Hz}, 2 \mathrm{H}), 3.33-$ $3.22(\mathrm{~m}, 2 \mathrm{H}), 2.12-1.99(\mathrm{~m}, 2 \mathrm{H}), 1.45(\mathrm{~s}, 9 \mathrm{H})$; MS (ESI, m/z) calculated for $\mathrm{C}_{8} \mathrm{H}_{16} \mathrm{BrNO}_{2} 237.0$, $[\mathrm{M}+\mathrm{H}]^{+}$found 238.8 .

Tert-butyl (3-(4-chloro-5-iodo-7H-pyrrolo[2,3-d]pyrimidin-7-yl)propyl)carbamate (5)

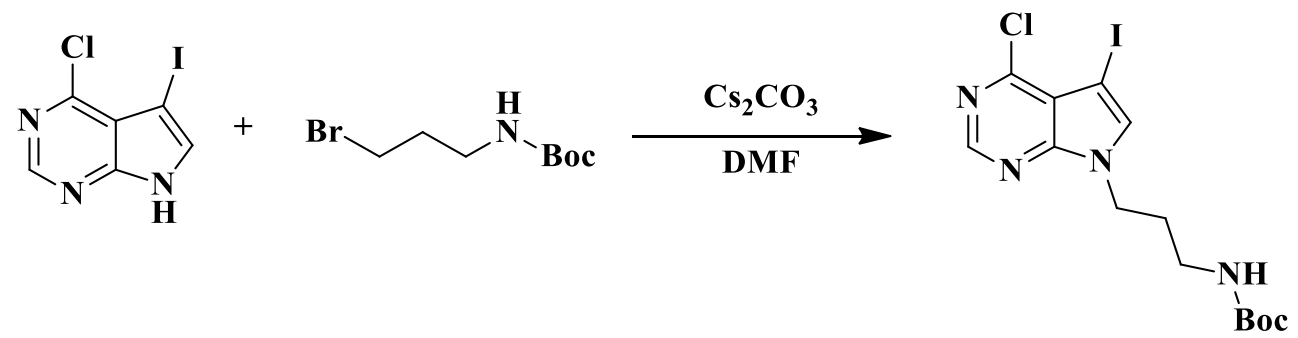

4-Chloro-5-iodo-7H-pyrrolo[2,3-d]pyrimidine (1.7 g, $6.2 \mathrm{mmol}, 1.0$ equiv.), tert-butyl (3bromopropyl)carbamate (2.0 g, $8.4 \mathrm{mmol}, 1.4$ equiv.) and cesium carbonate $(6.7 \mathrm{~g}, 12.4 \mathrm{mmol}, 2.0$ 
equiv.) were dissolved in dimethylformamide $(20 \mathrm{~mL})$ in a $100-\mathrm{mL}$ round bottom flask under nitrogen. The reaction mixture was heated at $50{ }^{\circ} \mathrm{C}$ for 14 hours. The solvent was then removed in vacuo and the resulting solid residue was redissolved in ethyl acetate $(200 \mathrm{~mL})$. The organic phase was washed with aqueous ammonium chloride solution $(40 \mathrm{~mL})$, aqueous sodium bicarbonate solution $(40 \mathrm{~mL})$, brine $(40 \mathrm{~mL})$, dried over anhydrous sodium sulfate and concentrated in vacuo. Purification with Flash chromatography afforded tert-butyl (3-(4-chloro-5-iodo-7H-pyrrolo[2,3d]pyrimidin-7-yl)propyl)carbamate as a pale yellow solid (1.6 g, 59\%). ${ }^{1} \mathrm{H}-\mathrm{NMR}$ (500 MHz, $\left.\mathrm{CDCl}_{3}\right) \delta=8.62(\mathrm{~s}, 1 \mathrm{H}), 7.45(\mathrm{~d}, \mathrm{~J}=14.2 \mathrm{~Hz}, 1 \mathrm{H}), 5.01(\mathrm{~s}, 1 \mathrm{H}), 4.33(\mathrm{t}, \mathrm{J}=6.6 \mathrm{~Hz}, 2 \mathrm{H}), 3.11-$ $3.03(\mathrm{~m}, 2 \mathrm{H}), 2.06-1.97(\mathrm{~m}, 2 \mathrm{H}), 1.45(\mathrm{~s}, 9 \mathrm{H}) ; \mathrm{MS}(\mathrm{ESI}, \mathrm{m} / \mathrm{z})$ calculated for $\mathrm{C}_{14} \mathrm{H}_{18} \mathrm{ClIN}_{4} \mathrm{O}_{2} 436.0$, $[\mathrm{M}+\mathrm{H}]^{+}$found 437.1 .

Tert-butyl (3-(4-chloro-6-formyl-5-iodo-7H-pyrrolo[2,3- $d]$ pyrimidin-7-yl)propyl)carbamate (6)
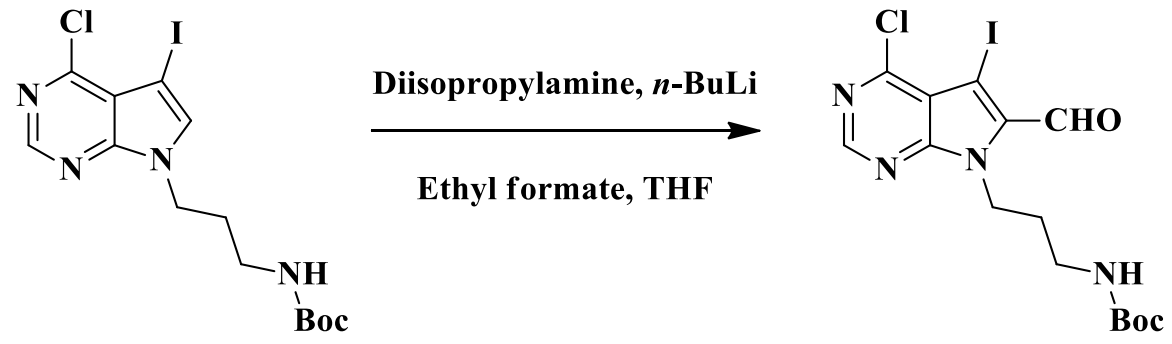

In a flame-dried 100-mL round bottom flask, diisopropylamine $(228 \mathrm{mg}, 316 \mu \mathrm{L}, 2.25 \mathrm{mmol}, 1.75$ equiv.) was dissolved in dry THF $(10 \mathrm{~mL})$ and cooled to $-78^{\circ} \mathrm{C}$ under nitrogen. $n$-BuLi solution (2.5 $\mathrm{M}$ in hexane, $901 \mu \mathrm{L}, 2.25 \mathrm{mmol}, 1.75$ equiv.) was added dropwise to the above solution at $78{ }^{\circ} \mathrm{C}$. The reaction was stirred at $0{ }^{\circ} \mathrm{C}$ for 1 hour and then cooled to $-78{ }^{\circ} \mathrm{C}$. A solution of tertbutyl (3-(4-chloro-5-iodo-7H-pyrrolo[2,3-d]pyrimidin-7-yl)propyl)carbamate (562 mg, 1.29 mmol, 1.00 equiv.) in dry THF $(5 \mathrm{~mL})$ was added dropwise at $-78^{\circ} \mathrm{C}$. After 1 hour, ethyl formate 
(200 mg, $218 \mu \mathrm{L}, 2.70 \mathrm{mmol}, 2.10$ equiv.) was added dropwise at $-78{ }^{\circ} \mathrm{C}$. The reaction mixture was allowed to warm up to room temperature overnight. The reaction was quenched by adding aqueous ammonium chloride solution $(2 \mathrm{~mL})$ and diluted with ethyl acetate $(150 \mathrm{~mL})$. The organic phase was washed with aqueous sodium bicarbonate solution $(20 \mathrm{~mL})$, brine $(20 \mathrm{~mL})$, dried over anhydrous sodium sulfate and concentrated in vacuo. Purification by flash chromatography on silica gel (EtOAc:Hexane= 0:100 to EtOAc: Hexane= 1:2) afforded tert-butyl (3-(4-chloro-6formyl-5-iodo-7H-pyrrolo[2,3- $d$ ]pyrimidin-7-yl)propyl)carbamate as a pale brown powder (130 $\mathrm{mg}, 22 \% ; \mathrm{R}_{\mathrm{f}}=0.57$ in EtOAc:Hexane= 3:2). ${ }^{1} \mathrm{H}-\mathrm{NMR}\left(300 \mathrm{MHz}, \mathrm{CDCl}_{3}\right) \delta=10.08(\mathrm{~s}, 1 \mathrm{H}), 8.77$ $(\mathrm{s}, 1 \mathrm{H}), 5.12(\mathrm{~s}, 1 \mathrm{H}), 4.72(\mathrm{t}, J=6.6 \mathrm{~Hz}, 2 \mathrm{H}), 3.14-2.96(\mathrm{~m}, 2 \mathrm{H}), 2.03-1.89(\mathrm{~m}, 2 \mathrm{H}), 1.45(\mathrm{~s}$, $9 \mathrm{H}) ; \mathrm{MS}(\mathrm{ESI}, \mathrm{m} / \mathrm{z})$ calculated for $\mathrm{C}_{15} \mathrm{H}_{18} \mathrm{ClIN}_{4} \mathrm{O}_{3} 464.0,[\mathrm{M}+\mathrm{H}]^{+}$found 465.0.

\section{Tert-butyl (3-(4-amino-6-formyl-5-iodo-7H-pyrrolo[2,3- $d]$ pyrimidin-7-yl)propyl)carbamate} (7)
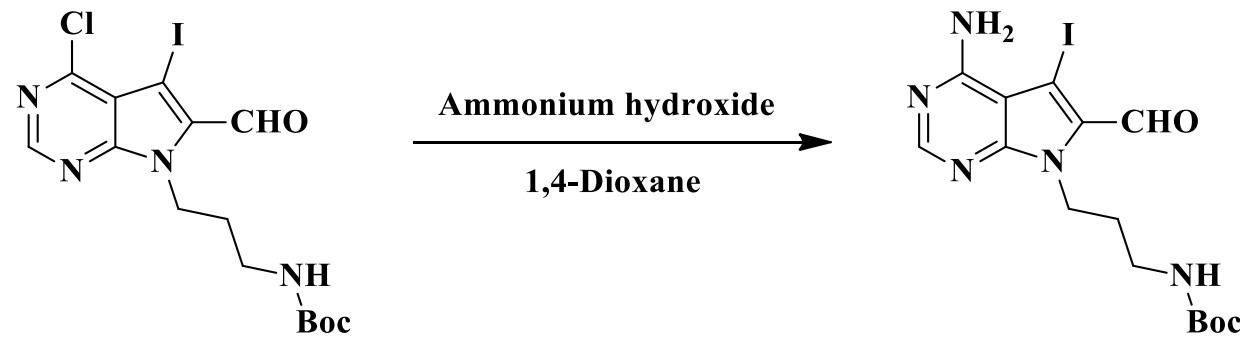

In a 10-mL pressure tube, tert-butyl (3-(4-chloro-6-formyl-5-iodo-7H-pyrrolo[2,3-d]pyrimidin-7yl)propyl)carbamate (130 mg, $0.28 \mathrm{mmol}, 1.0$ equiv.) was dissovled in 1,4-Dioxane (1.2 mL). To it was added aqueous ammonium hydroxide solution $\left(1.2 \mathrm{~mL}, 28.0 \%-30.0 \% \mathrm{NH}_{3}\right.$ basis $)$. The resulting solution was heated at $40{ }^{\circ} \mathrm{C}$ for 3 hours and then diluted with ethyl acetate $(40 \mathrm{~mL})$. The organic phase was washed with $1 \mathrm{M}$ hydrochloride aqueous solution, sodium bicarbonate, brine and dried over anhydrous sodium sulfate. Purification on silica gel using a gradiant of $\mathrm{MeOH}$ : 
$\mathrm{DCM}=0: 100$ to $\mathrm{MeOH}: \mathrm{DCM}=10: 90$ afforded tert-butyl (3-(4-amino-6-formyl-5-iodo-7Hpyrrolo[2,3-d]pyrimidin-7-yl)propyl)carbamate as a pale brown solid (54 $\mathrm{mg}, 43 \% ; \mathrm{R}_{\mathrm{f}}=0.51$ in MeOH:DCM= 1:9). ${ }^{1} \mathrm{H}-\mathrm{NMR}\left(300 \mathrm{MHz}, \mathrm{CDCl}_{3}\right) \delta=9.82(\mathrm{~s}, 1 \mathrm{H}), 8.38(\mathrm{~s}, 1 \mathrm{H}), 6.04(\mathrm{~s}, 2 \mathrm{H}), 5.50$ $(\mathrm{s}, 1 \mathrm{H}), 4.63(\mathrm{t}, \mathrm{J}=6.4 \mathrm{~Hz}, 2 \mathrm{H}), 3.07-2.91(\mathrm{~m}, 2 \mathrm{H}), 2.01-1.86(\mathrm{~m}, 2 \mathrm{H}), 1.45(\mathrm{~s}, 9 \mathrm{H}) ;$ MS (ESI, $\mathrm{m} / \mathrm{z}$ ) calculated for $\mathrm{C}_{15} \mathrm{H}_{20} \mathrm{IN}_{5} \mathrm{O}_{3} 445.1,[\mathrm{M}+\mathrm{H}]^{+}$found 446.3 .

\section{Tert-butyl(3-(4-amino-5-(4-chlorophenyl)-6-formyl-7H-pyrrolo[2,3- $d]$ pyrimidin-7-}

\section{yl)propyl)carbamate (8)}
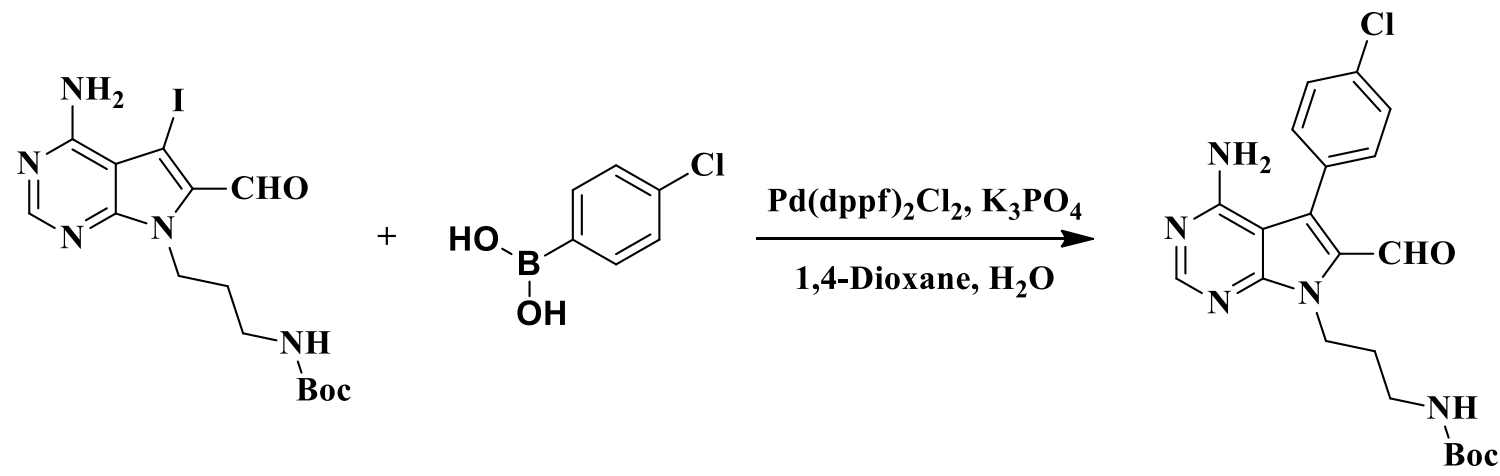

In a 10-mL microwave reaction tube, tert-butyl (3-(4-amino-6-formyl-5-iodo-7H-pyrrolo[2,3d]pyrimidin-7-yl)propyl)carbamate (54 $\mathrm{mg}, 0.12 \mathrm{mmol}, 1.0$ equiv.), 4-chlorophenylboronic acid (28 mg, $0.18 \mathrm{mmol}, 1.5$ equiv.), potassium phosphate (77 mg, $0.36 \mathrm{mmol}, 3.0$ equiv.) and [1,1'Bis(diphenylphosphino)ferrocene]dichloropalladium(II) (9.9 mg, $0.012 \mathrm{mmol}, 0.1$ equiv.) were dissolved in a mixture of 1,4-Dioxane $(1.0 \mathrm{~mL})$ and water $(0.25 \mathrm{~mL})$. The resulting mixture was heated in a microwave reactor at $90{ }^{\circ} \mathrm{C}$ for 130 mins. The reaction was then quenched with saturated $\mathrm{NH}_{4} \mathrm{Cl}$ solution $(1 \mathrm{~mL})$ and diluted with ethyl acetate $(30 \mathrm{~mL})$. The organic phase was washed with saturated $\mathrm{NaHCO} 3$ solution $(5 \mathrm{~mL})$, brine $(5 \mathrm{~mL})$, dried over anhydrous $\mathrm{Na}_{2} \mathrm{SO}_{4}$ and concentrated in vacuo. Purification using flash chromatography on silica gel with a EtOAc/Hexane gradient (0:100 to 90:10) afforded tert-butyl (3-(4-amino-5-(4-chlorophenyl)-6-formyl-7H- 
pyrrolo[2,3- $d$ ] pyrimidin-7-yl)propyl)carbamate as a pale yellow solid (39 $\mathrm{mg}, 74 \% ; \mathrm{Rf}=0.31$ in EtOAc:Hexane= 9:1). ${ }^{1} \mathrm{H}-\mathrm{NMR}\left(300 \mathrm{MHz}, \mathrm{CDCl}_{3}\right) \delta=9.60(\mathrm{~s}, 1 \mathrm{H}), 8.41(\mathrm{~s}, 1 \mathrm{H}), 7.58-7.42(\mathrm{~m}$, 4H), $5.62(\mathrm{~s}, 1 \mathrm{H}), 5.32(\mathrm{~s}, 2 \mathrm{H}), 4.70(\mathrm{t}, J=6.3 \mathrm{~Hz}, 2 \mathrm{H}), 3.19-2.94(\mathrm{~m}, 2 \mathrm{H}), 2.06-1.96(\mathrm{~m}, 2 \mathrm{H})$, 1.46 (s, 9H); MS (ESI, m/z) calculated for $\mathrm{C}_{21} \mathrm{H}_{24} \mathrm{ClN}_{5} \mathrm{O}_{3} 429.2$, [M+H] $]^{+}$found 430.1 .

\section{(Z)- or (E)-tert-butyl (3-(4-amino-5-(4-chlorophenyl)-6-(2-cyano-3-(isopropylamino)-3-} oxoprop-1-en-1-yl)-7H-pyrrolo[2,3-d]pyrimidin-7-yl)propyl)carbamate (9)

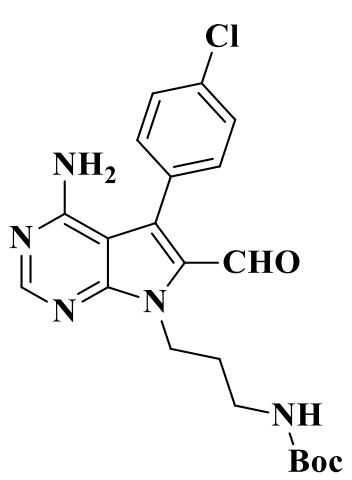

Tert-butyl

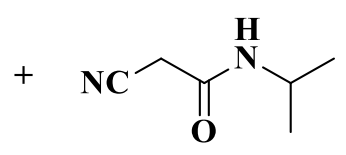

(3-(4-amino-5-(4-chlorophenyl)-6-formyl-7H-pyrrolo[2,3-d]pyrimidin-7-

yl)propyl)carbamate ( $20 \mathrm{mg}, 0.048 \mathrm{mmol}, 1.0$ equiv.) and 2-cyano- $N$-isopropylacetamide $(9.2 \mathrm{mg}$, $0.072 \mathrm{mmol}, 1.5$ equiv.) were dissolved in tetrahydrofuran $(300 \mu \mathrm{L})$. To the above solution was added 1,8-diazabicyclo[5.4.0]undec-7-ene ( $15 \mathrm{mg}, 14 \mu \mathrm{L}, 0.096 \mathrm{mmol}, 2.0$ equiv). The reaction mixture was stirred at room temperature for 6 hours. The reaction was then quenched with saturated $\mathrm{NH}_{4} \mathrm{Cl}$ solution $(1 \mathrm{~mL})$ and diluted with ethyl acetate $(30 \mathrm{~mL})$. The organic layer was washed with saturated $\mathrm{NaHCO} 3$ solution $(5 \mathrm{~mL})$, brine $(5 \mathrm{~mL})$, dried over anhydrous $\mathrm{Na}_{2} \mathrm{SO}_{4}$ and concentrated in vасио. Purification using preparative thin-layer chromatography developed by pure EtOAc rendered (Z)- or (E)-tert-butyl (3-(4-amino-5-(4-chlorophenyl)-6-(2-cyano-3(isopropylamino)-3-oxoprop-1-en-1-yl)-7H-pyrrolo[2,3- $d$ ]pyrimidin-7-yl)propyl)carbamate as a 
bright yellow powder (13 mg, 50\%; Rf [(E)- and (Z)-isomers) $]=0.25$ in EtOAc:Hexane= 9:1). ${ }^{1} \mathrm{H}$ NMR (300 MHz, MeOD) $\delta=8.30-8.18(\mathrm{~m}, 3 \mathrm{H}), 7.70(\mathrm{~s}, 1 \mathrm{H}), 7.64-7.36(\mathrm{~m}, 8 \mathrm{H}), 6.68(\mathrm{~s}, 2 \mathrm{H})$, $5.53(\mathrm{~s}, 2 \mathrm{H}), 5.16(\mathrm{~s}, 4 \mathrm{H}), 4.43(\mathrm{t}, J=6.9 \mathrm{~Hz}, 2 \mathrm{H}), 4.34(\mathrm{t}, J=7.0 \mathrm{~Hz}, 2 \mathrm{H}), 4.19-4.04(\mathrm{~m}, 1 \mathrm{H})$, $3.71-3.54(\mathrm{~m}, 1 \mathrm{H}), 3.15-2.97(\mathrm{~m}, 4 \mathrm{H}), 2.06-1.92(\mathrm{~m}, 4 \mathrm{H}), 1.46(\mathrm{~s}, 9 \mathrm{H}), 1.33(\mathrm{~s}, 9 \mathrm{H}), 1.24(\mathrm{~d}$, $J=6.6 \mathrm{~Hz}, 6 \mathrm{H}), 1.05(\mathrm{~d}, J=6.6 \mathrm{~Hz}, 6 \mathrm{H}) ; \mathrm{MS}(\mathrm{ESI}, \mathrm{m} / \mathrm{z})$ calculated for $\mathrm{C}_{27} \mathrm{H}_{32} \mathrm{ClN}_{7} \mathrm{O}_{3}$ 537.2, $[\mathrm{M}+\mathrm{H}]^{+}$found 538.1.

(E)-cyclooct-4-en-1-yl (3-(4-amino-5-(4-chlorophenyl)-6-((E)-2-cyano-3-(isopropylamino)-3oxoprop-1-en-1-yl)-7H-pyrrolo[2,3- $d$ ]pyrimidin-7-yl)propyl)carbamate or (E)-cyclooct-4en-1-yl (3-(4-amino-5-(4-chlorophenyl)-6-((Z)-2-cyano-3-(isopropylamino)-3-oxoprop-1-en1-yl)-7H-pyrrolo[2,3- $d]$ pyrimidin-7-yl)propyl)carbamate (1-TCO)
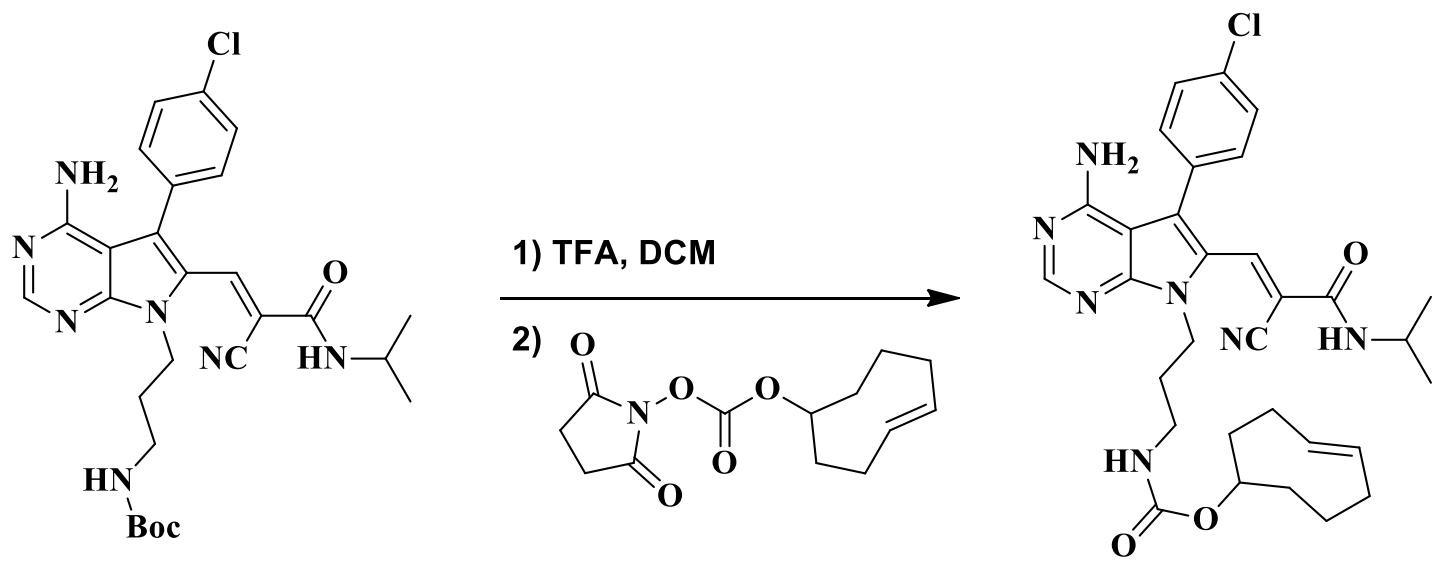

(Z)- or (E)-tert-butyl (3-(4-amino-5-(4-chlorophenyl)-6-(2-cyano-3-(isopropylamino)-3-oxoprop1-en-1-yl)-7H-pyrrolo[2,3-d]pyrimidin-7-yl)propyl)carbamate (13 mg, $0.024 \mathrm{mmol}, 1.0$ equiv.) was dissolved in a mixture of trifluoacetic acid $(0.30 \mathrm{~mL})$ and dichloromethane $(0.70 \mathrm{~mL})$. The reaction solution was stirred at room temperature for $30 \mathrm{mins}$ and concentrated in vacuo. The solid residue was dissolved in anhydrous tetrahydrofuran $(400 \mu \mathrm{L})$. Then diisopropylethylamine $(7.8$ $\mathrm{mg}, 11 \mu \mathrm{L}, 0.060 \mathrm{mmol}, 2.5$ equiv.) and (E)-cyclooct-4-en-1-yl (2,5-dioxopyrrolidin-1-yl) 
carbonate $(8.4 \mathrm{mg}, 0.031 \mathrm{mmol}, 1.3$ equiv.) were added to the above solution sequentially. The resulting mixture was stirred at room temperature overnight and quenched with water $(1 \mathrm{~mL})$ and diluted with ethyl acetate $(30 \mathrm{~mL})$. The organic layer was washed with saturated $\mathrm{NaHCO}_{3}$ solution $(5 \mathrm{~mL})$, brine $(5 \mathrm{~mL})$, dried over anhydrous $\mathrm{Na}_{2} \mathrm{SO}_{4}$ and concentrated in vacuo. Purification using flash chromatography with a EtOAc/Hexane gradient (0:100 to 90:10) afforded a mixture of $(E)$ cyclooct-4-en-1-yl (3-(4-amino-5-(4-chlorophenyl)-6-((E)-2-cyano-3-(isopropylamino)-3oxoprop-1-en-1-yl)-7H-pyrrolo[2,3-d]pyrimidin-7-yl)propyl)carbamate or (E)-cyclooct-4-en-1-yl (3-(4-amino-5-(4-chlorophenyl)-6-((Z)-2-cyano-3-(isopropylamino)-3-oxoprop-1-en-1-yl)-7Hpyrrolo[2,3-d]pyrimidin-7-yl)propyl)carbamate as a bright yellow powder $(8.6 \mathrm{mg}, 60 \%$ for 2 steps; $\mathrm{R}_{\mathrm{f}}[(E)-$ and $(Z)$-isomers $\left.)\right]=0.33$ or 0.26 in EtOAc:Hexane= 9:1) ${ }^{1} \mathrm{H}-\mathrm{NMR}(300 \mathrm{MHz}$, $\mathrm{MeOD}) \delta=8.26-8.16(\mathrm{~m}, 3 \mathrm{H}), 7.66(\mathrm{~s}, 1 \mathrm{H}), 7.60-7.34(\mathrm{~m}, 8 \mathrm{H}), 6.77(\mathrm{~s}, 2 \mathrm{H}), 5.76-5.36(\mathrm{~m}$, $4 \mathrm{H}), 5.13(\mathrm{~s}, 4 \mathrm{H}), 4.45-4.21(\mathrm{~m}, 4 \mathrm{H}), 4.18-4.00(\mathrm{~m}, 1 \mathrm{H}), 3.70-3.49(\mathrm{~m}, 1 \mathrm{H}), 3.17-2.92(\mathrm{~m}$, 4H), $2.43-2.27(\mathrm{~m}, 4 \mathrm{H}), 2.27-0.94(\mathrm{~m}, 32 \mathrm{H}), 1.00-0.82(\mathrm{~m}, 4 \mathrm{H}) ; \mathrm{MS}(\mathrm{ESI}, \mathrm{m} / \mathrm{z})$ calculated for $\mathrm{C}_{31} \mathrm{H}_{36} \mathrm{ClN}_{7} \mathrm{O}_{3} \mathrm{H}(\mathrm{M}+\mathrm{H})$ 590.2641, [M+H] $]^{+}$(HRMS) found 590.2633; HPLC purity: 97.9\% (UV detector wavelength: $254 \mathrm{~nm})$. 


\section{Synthesis of 2-TCO}
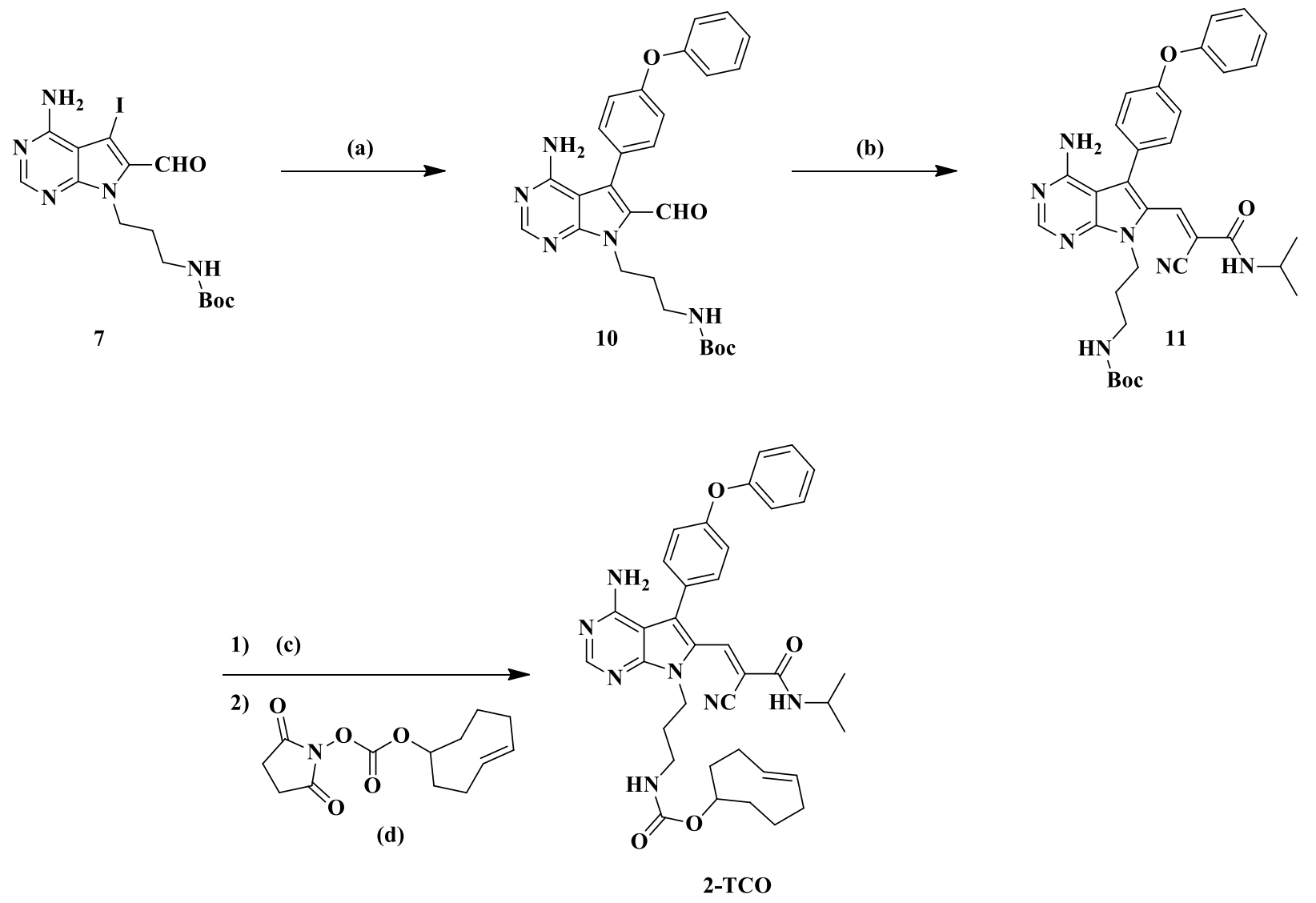

Reaction scheme for 2-TCO: (a) 4-Phenoxyphenylboronic acid, $\mathrm{K}_{3} \mathrm{PO}_{4}, \operatorname{Pd}(\mathrm{dppf})_{2} \mathrm{Cl}_{2}, 1,4-$

Dioxane, $\mathrm{H}_{2} \mathrm{O}, 90{ }^{\circ} \mathrm{C}, 150$ mins (microwave), 72\%; (b) 2-Cyano- $N$-isopropylacetamide, DBU, THF, rt, 80 mins, 57\%; (c) TFA, DCM, rt, 30 mins; (d) DIPEA, THF, rt, overnight, $44 \%$. 

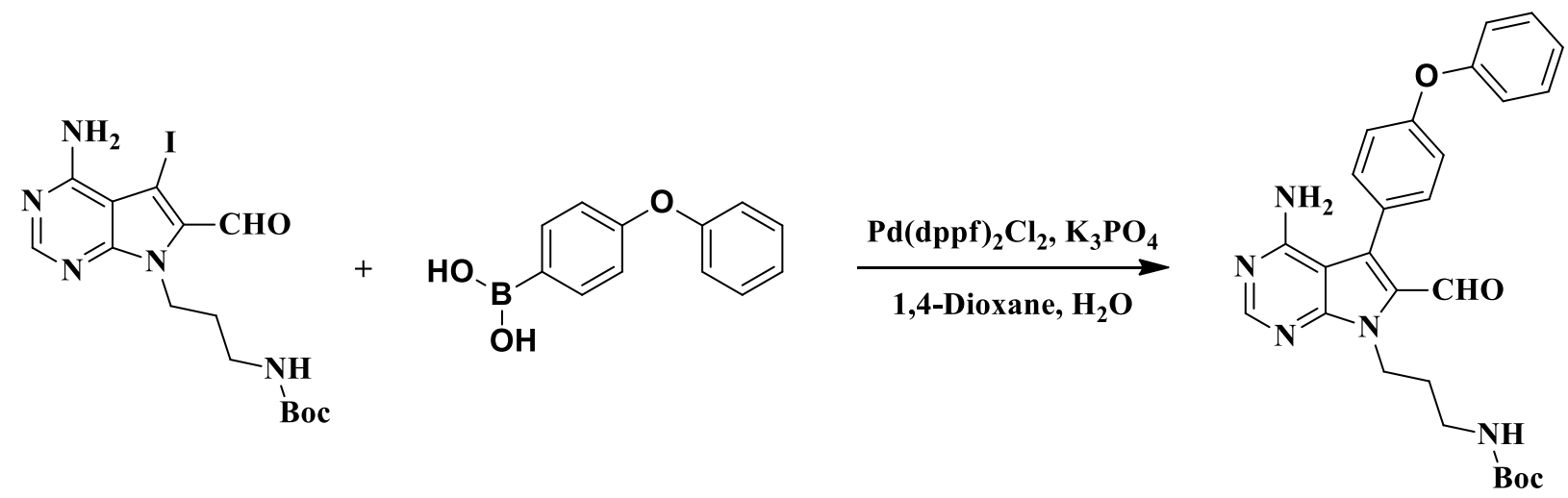

In a $10-\mathrm{mL}$ microwave reaction tube, tert-butyl (3-(4-amino-6-formyl-5-iodo-7 $\mathrm{H}$-pyrrolo[2,3d]pyrimidin-7-yl)propyl)carbamate (72 mg, $0.16 \mathrm{mmol}, 1.0$ equiv.), 4-phenoxyphenylboronic acid (52 mg, $0.24 \mathrm{mmol}, 1.5$ equiv.), potassium phosphate (100 mg, $0.49 \mathrm{mmol}, 3.0$ equiv.) and [1,1'Bis(diphenylphosphino)ferrocene]dichloropalladium(II) (13 $\mathrm{mg}, 0.016 \mathrm{mmol}, 0.1$ equiv.) were dissolved in a mixture of 1,4-Dioxane $(1.3 \mathrm{~mL})$ and water $(0.33 \mathrm{~mL})$. The resulting mixture was heated in a microwave reactor at $90{ }^{\circ} \mathrm{C}$ for 150 mins. The reaction was quenched with saturated $\mathrm{NH}_{4} \mathrm{Cl}$ solution $(1 \mathrm{~mL})$ and diluted with ethyl acetate $(30 \mathrm{~mL})$. The organic phase was washed with saturated $\mathrm{NaHCO} 3$ solution $(5 \mathrm{~mL})$, brine $(5 \mathrm{~mL})$, dried over anhydrous $\mathrm{Na}_{2} \mathrm{SO}_{4}$ and concentrated in vacuo. Purification using flash chromatography on silica gel with a EtOAc/Hexane gradient (0:100 to 90:10) afforded tert-butyl (3-(4-amino-6-formyl-5-(4-phenoxyphenyl)-7H-pyrrolo[2,3d]pyrimidin-7-yl)propyl)carbamate as a pale yellow solid $\left(57 \mathrm{mg}, 72 \% ; \mathrm{R}_{\mathrm{f}}=0.87\right.$ in EtOAc:Hexane= 9:1). ${ }^{1} \mathrm{H}-\mathrm{NMR}\left(500 \mathrm{MHz}, \mathrm{CDCl}_{3}\right) \delta=9.64(\mathrm{~s}, 1 \mathrm{H}), 8.42(\mathrm{~s}, 1 \mathrm{H}), 7.48-7.38(\mathrm{~m}$, 4H), $7.21(\mathrm{t}, \mathrm{J}=7.4 \mathrm{~Hz}, 1 \mathrm{H}), 7.16-7.09(\mathrm{~m}, 4 \mathrm{H}), 5.68(\mathrm{~s}, 1 \mathrm{H}), 5.35(\mathrm{~s}, 2 \mathrm{H}), 4.69(\mathrm{t}, \mathrm{J}=6.3 \mathrm{~Hz}$, 2H), $3.10-2.98(\mathrm{~m}, 2 \mathrm{H}), 2.05-1.96(\mathrm{~m}, 2 \mathrm{H}), 1.46(\mathrm{~s}, 9 \mathrm{H})$; MS (ESI, m/z) calculated for $\mathrm{C}_{27} \mathrm{H}_{29} \mathrm{~N}_{5} \mathrm{O}_{4} 487.2,[\mathrm{M}+\mathrm{H}]^{+}$found 488.5. 
(Z)- or (E)-tert-butyl (3-(4-amino-6-(2-cyano-3-(isopropylamino)-3-oxoprop-1-en-1-yl)-5-(4phenoxyphenyl)-7H-pyrrolo[2,3- $d]$ pyrimidin-7-yl)propyl)carbamate (11)
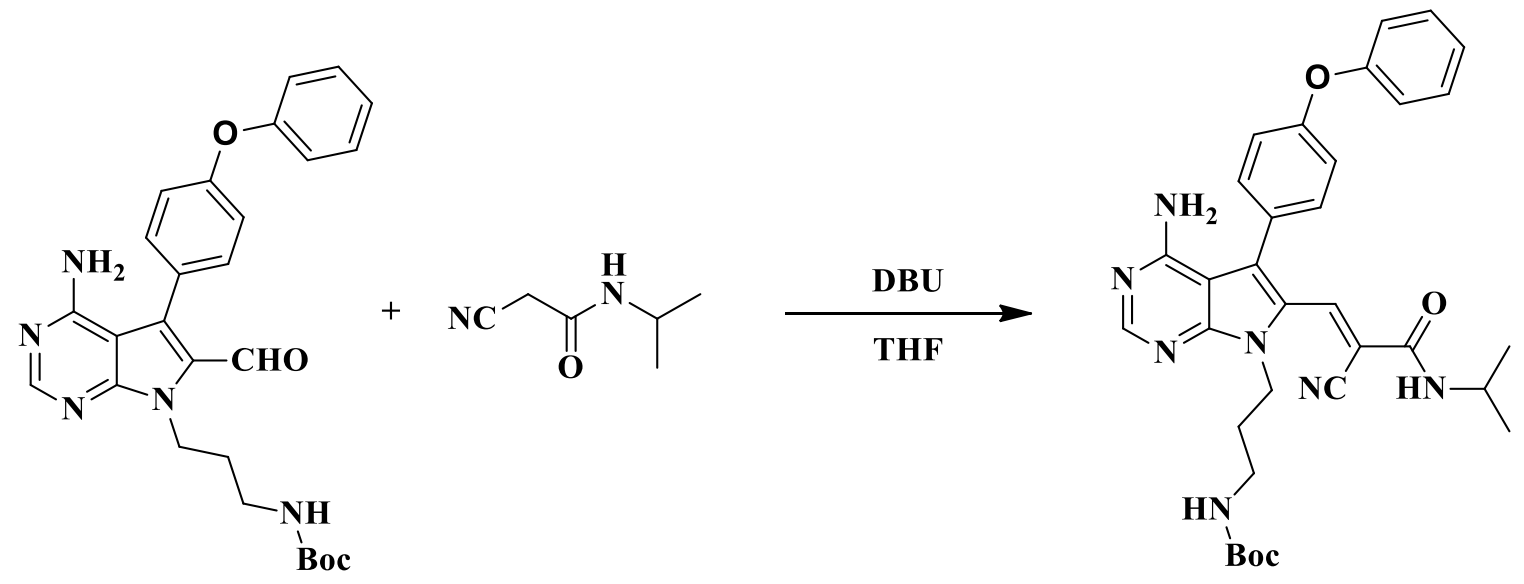

Tert-butyl (3-(4-amino-6-formyl-5-(4-phenoxyphenyl)-7H-pyrrolo[2,3-d]pyrimidin-7yl)propyl)carbamate (57 mg, $0.095 \mathrm{mmol}, 1.0$ equiv.) and 2-cyano- $N$-isopropylacetamide (18 mg, $0.14 \mathrm{mmol}, 1.5$ equiv.) were dissolved in dry THF $(600 \mu \mathrm{L} \mathrm{mL}) .1,8$-Diazabicyclo[5.4.0]undec-7ene (29 mg, $28 \mu \mathrm{L}, 0.19 \mathrm{mmol}, 2.0$ equiv.) was added and the resulting solution was stirred at room temperature for 80 mins. The reaction mixture was then diluted with EtOAc $(30 \mathrm{~mL})$ and quenched with saturated aqueous $\mathrm{NH}_{4} \mathrm{Cl}$ solution. The organic phase was washed with saturated $\mathrm{NaHCO}_{3}$ solution $(5 \mathrm{~mL})$, brine $(5 \mathrm{~mL})$, dried over anhydrous $\mathrm{Na}_{2} \mathrm{SO}_{4}$. Purification with preparative thin-layer chromatography afforded a mixture of (Z)- or (E)-tert-butyl (3-(4-amino-6(2-cyano-3-(isopropylamino)-3-oxoprop-1-en-1-yl)-5-(4-phenoxyphenyl)-7H-pyrrolo[2,3d]pyrimidin-7-yl)propyl)carbamate as a bright yellow powder (32 mg, 57\%; Rf [(E) and (Z)isomers) $]=0.26$ in EtOAc:Hexane= 9:1). ${ }^{1} \mathrm{H}-\mathrm{NMR}(300 \mathrm{MHz}, \mathrm{MeOD}) \delta=8.25-8.19(\mathrm{~m}, 3 \mathrm{H})$, $7.65(\mathrm{~s}, 1 \mathrm{H}), 7.51-7.34(\mathrm{~m}, 8 \mathrm{H}), 7.24-7.05(\mathrm{~m}, 10 \mathrm{H}), 5.14(\mathrm{~s}, 2 \mathrm{H}), 4.48-4.38(\mathrm{~m}, 2 \mathrm{H}), 4.31(\mathrm{t}$, $J=7.0 \mathrm{~Hz}, 2 \mathrm{H}), 4.15-4.07(\mathrm{~m}, 1 \mathrm{H}), 3.74-3.62(\mathrm{~m}, 1 \mathrm{H}), 3.13-2.94(\mathrm{~m}, 4 \mathrm{H}), 2.04-1.85(\mathrm{~m}$, 
4H), $1.44(\mathrm{~s}, 18 \mathrm{H}), 1.23(\mathrm{~d}, J=6.6 \mathrm{~Hz}, 6 \mathrm{H}), 1.07(\mathrm{~d}, J=6.5 \mathrm{~Hz}, 6 \mathrm{H}) ; \mathrm{MS}(\mathrm{ESI}, \mathrm{m} / \mathrm{z})$ calculated for $\mathrm{C}_{33} \mathrm{H}_{37} \mathrm{~N}_{7} \mathrm{O}_{4}$ 595.3, $[\mathrm{M}+\mathrm{H}]^{+}$found 597.4.

(E)-cyclooct-4-en-1-yl (3-(4-amino-6-((E)-2-cyano-3-(isopropylamino)-3-oxoprop-1-en-1-yl)5-(4-phenoxyphenyl)-7H-pyrrolo[2,3-d]pyrimidin-7-yl)propyl)carbamate or (E)-cyclooct-4en-1-yl (3-(4-amino-6-((Z)-2-cyano-3-(isopropylamino)-3-oxoprop-1-en-1-yl)-5-(4phenoxyphenyl)-7H-pyrrolo[2,3- $d]$ pyrimidin-7-yl)propyl)carbamate (2-TCO)
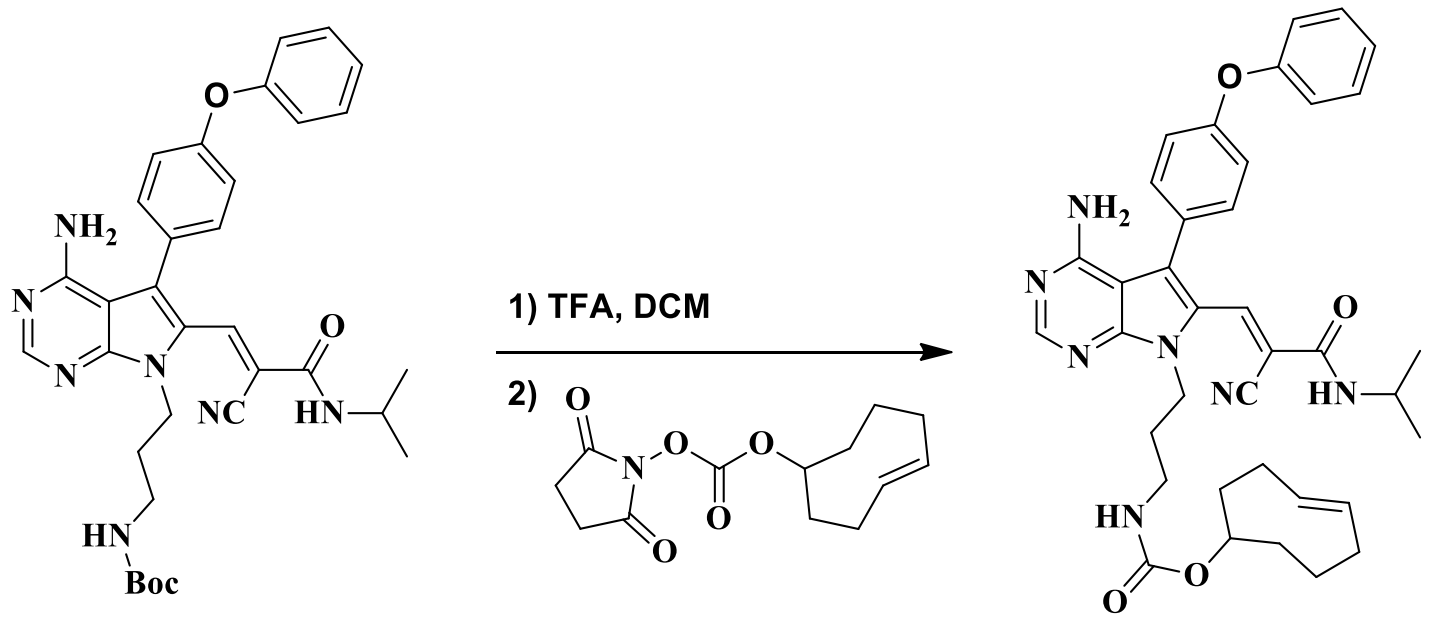

A mixture of (Z)- or (E)-tert-butyl (3-(4-amino-6-(2-cyano-3-(isopropylamino)-3-oxoprop-1-en1-yl)-5-(4-phenoxyphenyl)-7H-pyrrolo[2,3-d]pyrimidin-7-yl)propyl)carbamate $(24 \mathrm{mg}, 0.034$ mmol, 1.0 equiv.) was dissolved in a mixture of trifluoacetic acid $(0.60 \mathrm{~mL})$ and dichloromethane $(1.4 \mathrm{~mL})$. The resulting soluting was stirred at room temperature for 30 mins and concentrated in vacuo. The solid residue was dissolved in anhydrous tetrahydrofuran $(2 \mathrm{~mL})$, and to it was added diisopropylethylamine (22 mg, $30 \mu \mathrm{L}, 0.17 \mathrm{mmol}, 5.0$ equiv.) and (E)-cyclooct-4-en-1-yl (2,5dioxopyrrolidin-1-yl) carbonate (12 $\mathrm{mg}, 0.044 \mathrm{mmol}, 1.3$ equiv.) sequentially. The resulting mixture was stirred at room temperature overnight and quenched with water $(1 \mathrm{~mL})$ and diluted with ethyl acetate $(30 \mathrm{~mL})$. The organic layer was washed with saturated $\mathrm{NaHCO} 3$ solution (5 
$\mathrm{mL})$, brine $(5 \mathrm{~mL})$, dried over anhydrous $\mathrm{Na} 2 \mathrm{SO} 4$. The crude product was purified using flash chromatography with a $\mathrm{MeOH} / \mathrm{EtOAc}$ gradient (0:100 to 10:90). A mixture of isomers of $(E)$ cyclooct-4-en-1-yl (3-(4-amino-6-((E)-2-cyano-3-(isopropylamino)-3-oxoprop-1-en-1-yl)-5-(4phenoxyphenyl)-7H-pyrrolo[2,3-d]pyrimidin-7-yl)propyl)carbamate or (E)-cyclooct-4-en-1-yl (3(4-amino-6-((Z)-2-cyano-3-(isopropylamino)-3-oxoprop-1-en-1-yl)-5-(4-phenoxyphenyl)-7Hpyrrolo[2,3- $d$ pyrimidin-7-yl)propyl)carbamate was rendered as a bright yellow powder $(9.6 \mathrm{mg}$, $44 \%$ for 2 steps; $\operatorname{Rf}[(\mathrm{E})$ and $(\mathrm{Z})$-isomers) $]=0.27$ in EtOAc:Hexane $=3: 2){ }^{1} \mathrm{H}-\mathrm{NMR}(300 \mathrm{MHz}$, MeOD) $\delta=8.26-8.13(\mathrm{~m}, 3 \mathrm{H}), 7.60(\mathrm{~s}, 1 \mathrm{H}), 7.50-7.32(\mathrm{~m}, 8 \mathrm{H}), 7.23-7.05(\mathrm{~m}, 10 \mathrm{H}), 5.52(\mathrm{t}$, $4 \mathrm{H}), 4.40(\mathrm{t}, \mathrm{J}=6.8 \mathrm{~Hz}, 2 \mathrm{H}), 4.27(\mathrm{t}, \mathrm{J}=6.8 \mathrm{~Hz}, 2 \mathrm{H}), 4.19-4.02(\mathrm{~m}, 1 \mathrm{H}), 3.77-3.59(\mathrm{~m}, 1 \mathrm{H})$, $3.27-3.14(\mathrm{~m}, 2 \mathrm{H}), 3.14-2.93(\mathrm{~m}, 4 \mathrm{H}), 2.42-2.22(\mathrm{~m}, 4 \mathrm{H}), 2.22-0.97(\mathrm{~m}, 32 \mathrm{H}), 0.97-0.81$ (m, 4H); MS (ESI, m/z) calculated for $\mathrm{C}_{37} \mathrm{H}_{41} \mathrm{~N}_{7} \mathrm{O}_{4} \mathrm{H}(\mathrm{M}+\mathrm{H})$ 648.3293, [M+H] $]^{+}$(HRMS) found 648.3279; HPLC purity: 99.0\% (UV detector wavelength: $254 \mathrm{~nm}$ ) 


\section{Synthesis of 3-TCO}
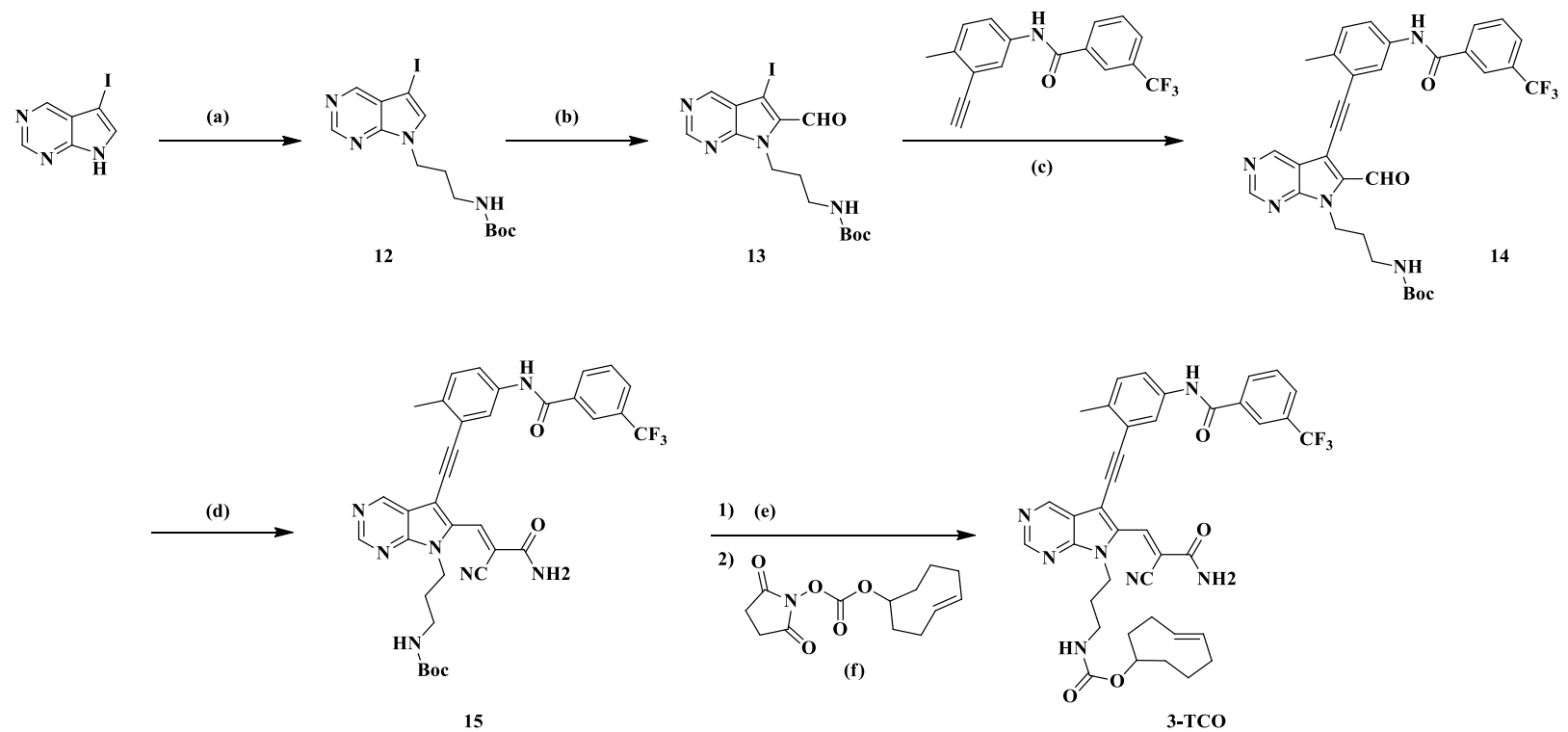

Reaction scheme for 3-TCO: (a) $\mathrm{Cs}_{2} \mathrm{CO}_{3}$, DMF, $50{ }^{\circ} \mathrm{C}$, overnight, 73\%; (b) Diisopropylamine, $n$ BuLi, ethyl formate, THF, $-78{ }^{\circ} \mathrm{C}$-rt, overnight, $32 \%$; (c) $N$-(3-ethynyl-4-methylphenyl)-3(trifluoromethyl)-benzamide, $\quad \mathrm{Pd}\left(\mathrm{PPh}_{3}\right)_{2} \mathrm{Cl}_{2}, \quad \mathrm{CuI}, \quad \mathrm{DMF}, \quad 50 \quad{ }^{\circ} \mathrm{C}$, overnight, $39 \%$; (f) Cyanoacetamide, DBU, THF, rt, 25 mins, 44\%; (g) TFA, DCM, rt, 30 mins; (h) DIPEA, THF, rt, 30 mins, $59 \%$.

Tert-butyl (3-(5-iodo-7H-pyrrolo[2,3-d]pyrimidin-7-yl)propyl)carbamate (12)

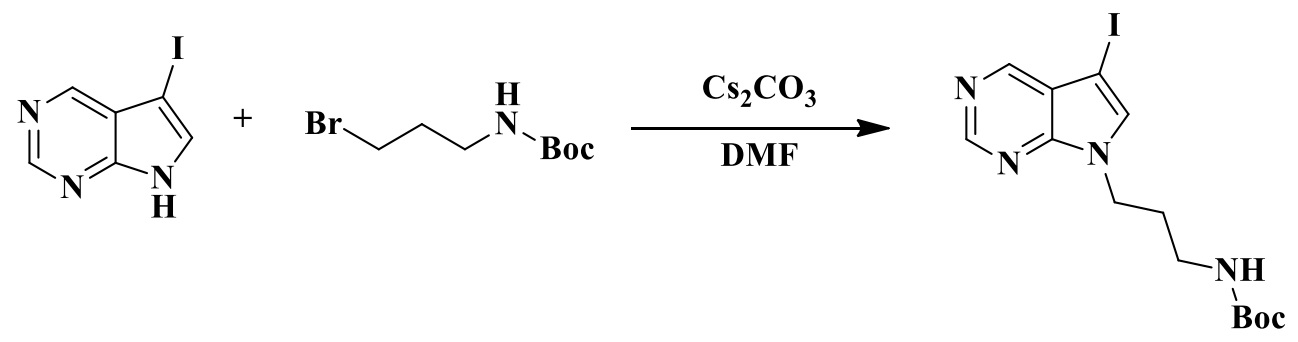

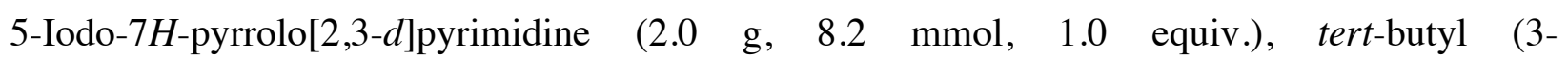
bromopropyl)carbamate $(2.5 \mathrm{~g}, 11 \mathrm{mmol}, 1.3$ equiv. $)$ and cesium carbonate $(8.8 \mathrm{~g}, 16 \mathrm{mmol}, 2.0$ equiv.) were dissolved in dimethylformamide $(20 \mathrm{~mL})$ in a $100-\mathrm{mL}$ round bottom flask. The 
reaction mixture was heated at $50{ }^{\circ} \mathrm{C}$ overnight. The solvent was then removed in vacuo. The crude compound was redissolved in ethyl acetate $(200 \mathrm{~mL})$ and washed with saturated $\mathrm{NH}_{4} \mathrm{Cl}$ solution $(40 \mathrm{~mL})$, aqueous $\mathrm{NaHCO}_{3}$ solution $(40 \mathrm{~mL})$, brine $(40 \mathrm{~mL})$, dried over anhydrous $\mathrm{Na}_{2} \mathrm{SO}_{4}$. Purification with Flash chromatography afforded tert-butyl (3-(5-iodo-7H-pyrrolo[2,3d]pyrimidin-7-yl)propyl)carbamate as a pale brown solid $(2.4 \mathrm{~g}, 73 \% ; \mathrm{Rf}=0.20$ in EtOAc:Hexane= 1:2). ${ }^{1} \mathrm{H}-\mathrm{NMR}\left(300 \mathrm{MHz}, \mathrm{CDCl}_{3}\right) \delta=8.81(\mathrm{~s}, 1 \mathrm{H}), 8.66(\mathrm{~s}, 1 \mathrm{H}), 7.16(\mathrm{~s}, 1 \mathrm{H})$, $5.02(\mathrm{~s}, 1 \mathrm{H}), 4.25(\mathrm{t}, J=6.5 \mathrm{~Hz}, 2 \mathrm{H}), 3.13-2.82(\mathrm{~m}, 2 \mathrm{H}), 2.09-1.82(\mathrm{~m}, 2 \mathrm{H}), 1.36(\mathrm{~s}, 9 \mathrm{H}) ; \mathrm{MS}$ (ESI, m/z) calculated for $\mathrm{C}_{14} \mathrm{H}_{19} \mathrm{IN}_{4} \mathrm{O}_{2} 402.1,[\mathrm{M}+\mathrm{H}]^{+}$found 403.1 .

\section{Tert-butyl (3-(6-formyl-5-iodo-7H-pyrrolo[2,3-d]pyrimidin-7-yl)propyl)carbamate (13)}
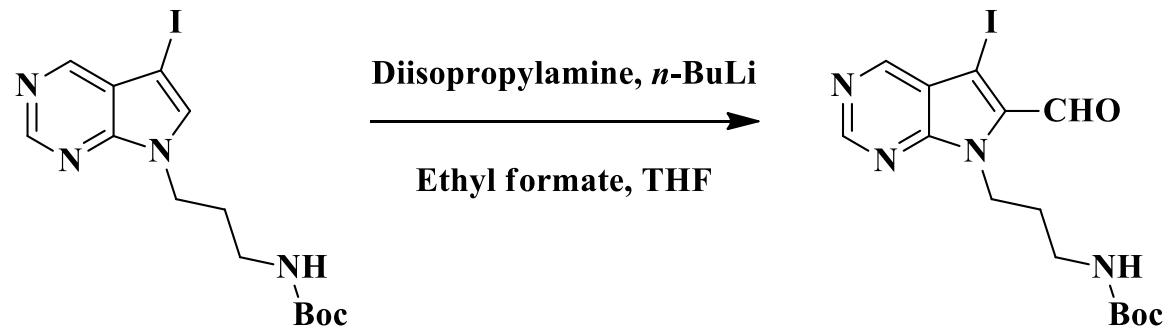

Diisopropylamine (490 mg, $680 \mu \mathrm{L}, 4.9 \mathrm{mmol}, 2.7$ equiv.) was dissolved in dry THF (6 mL) and cooled to $-78^{\circ} \mathrm{C}$. A solution of $n$ - $\mathrm{BuLi}(2.5 \mathrm{M}$ in hexane, $1.8 \mathrm{~mL}, 4.5 \mathrm{mmol}, 2.5$ equiv.) was added dropwise to the above solution at $-78{ }^{\circ} \mathrm{C}$. The solution was stirred at $0{ }^{\circ} \mathrm{C}$ for 1 hour and then cooled to $-78{ }^{\circ} \mathrm{C}$. A solution of tert-butyl (3-(5-iodo-7H-pyrrolo[2,3- $\left.d\right]$ pyrimidin-7yl)propyl)carbamate (730 mg, $1.8 \mathrm{mmol}, 1.0$ equiv., dissolved in $3 \mathrm{~mL}$ dry THF) was added dropwise and the reaction mixture was then stirred at $-78^{\circ} \mathrm{C}$ for 1 hour. A solution of ethyl formate (536 mg, $580 \mu \mathrm{L}, 7.2 \mathrm{mmol}, 4.0$ equiv., dissolved in $3 \mathrm{~mL}$ dry THF) was then added dropwise at $-78^{\circ} \mathrm{C}$. The reaction was allowed to warm to room temperature overnight. After reaction, saturated $\mathrm{NH} 4 \mathrm{Cl}$ solution $(2 \mathrm{~mL})$ was added and the resulting mixture was diluted with ethyl acetate (200 
$\mathrm{mL}$ ). The organic layer was washed with saturated aqueous $\mathrm{NaHCO} 3$ solution, brine and dried over anhydrous Na2SO4. Purification by flash chromatography on silica gel (EtOAc: Hexane= 0:100 to EtOAc: Hexane= 3:2) afforded tert-butyl (3-(6-formyl-5-iodo-7H-pyrrolo[2,3d]pyrimidin-7-yl)propyl)carbamate as a pale brown solid (250 mg, 32\%). ${ }^{1} \mathrm{H}-\mathrm{NMR}(300 \mathrm{MHz}$, $\left.\mathrm{CDCl}_{3}\right) \delta=10.02(\mathrm{~s}, 1 \mathrm{H}), 9.07(\mathrm{~s}, 1 \mathrm{H}), 8.98(\mathrm{~s}, 1 \mathrm{H}), 5.22(\mathrm{~s}, 1 \mathrm{H}), 4.73(\mathrm{t}, J=6.6 \mathrm{~Hz}, 2 \mathrm{H}), 3.13-$ $2.96(\mathrm{~m}, 2 \mathrm{H}), 2.10-1.91(\mathrm{~m}, 2 \mathrm{H}), 1.45(\mathrm{~s}, 9 \mathrm{H})$. MS (ESI, m/z) calculated for $\mathrm{C}_{15} \mathrm{H}_{19} \mathrm{IN}_{4} \mathrm{O}_{3} 430.1$, $[\mathrm{M}+\mathrm{H}]^{+}$found 431.0 .

Tert-butyl (3-(6-formyl-5-((2-methyl-5-(3-(trifluoromethyl)benzamido)phenyl)ethynyl)-7Hpyrrolo[2,3- $d]$ pyrimidin-7-yl)propyl)carbamate (14)

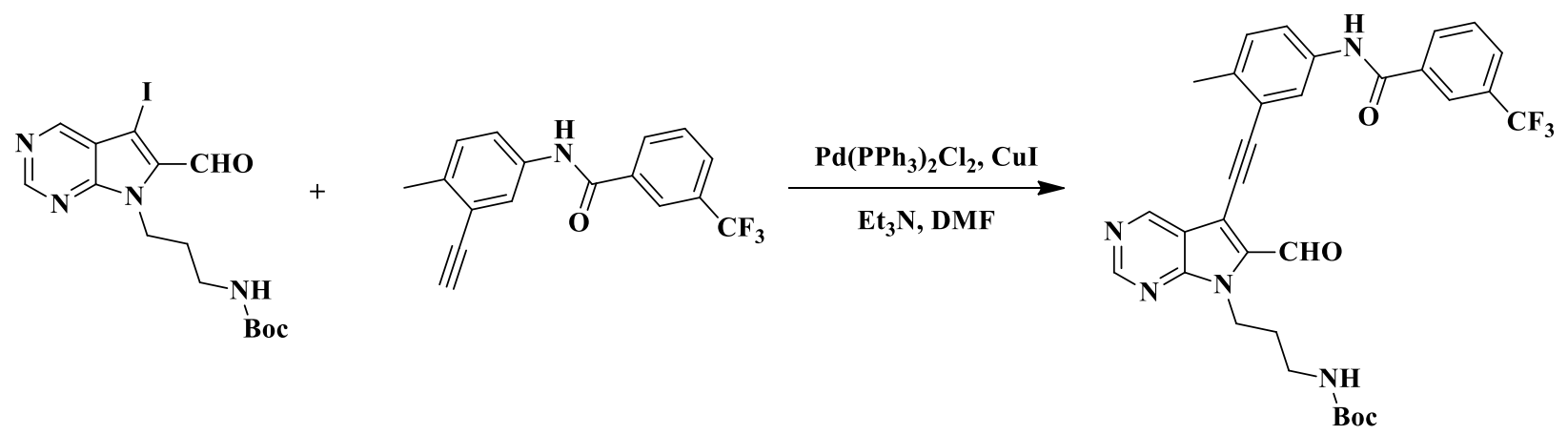

$N$-(3-ethynyl-4-methylphenyl)-3-(trifluoromethyl)-benzamide was synthesized according to previous reported (ref. SFK paper). Tert-butyl (3-(6-formyl-5-iodo-7H-pyrrolo[2,3-d]pyrimidin7-yl)propyl)carbamate (120 mg, $0.28 \mathrm{mmol}, 1.0$ equiv.) was dissovled in anhydrous DMF (3.5 mL) under nitrogen. Triethylamine (110 mg, $153 \mu \mathrm{L}, 1.1 \mathrm{mmol}, 4.0$ equiv.), N-(3-ethynyl-4methylphenyl)-3-(trifluoromethyl)benzamide $\quad(130 \quad \mathrm{mg}, \quad 0.42 \quad \mathrm{mmol}, \quad 1.5 \quad$ equiv.), bis(triphenylphosphine)palladium(II) dichloride (9.8 mg, $0.014 \mathrm{mmol}, 0.05$ equiv.) and copper (I) iodide (5.3 mg, $0.028 \mathrm{mmol}, 0.1$ equiv.) were added to the above solution sequentially. The 
reaction was heated at $50{ }^{\circ} \mathrm{C}$ for overnight and then quenched with saturated $\mathrm{NH} 4 \mathrm{Cl}(1 \mathrm{~mL})$. The resulting mixture was diluted with ethyl acetate $(200 \mathrm{~mL})$ and the organic phase was washed with saturated NaHCO3 $(30 \mathrm{~mL})$, brine $(30 \mathrm{~mL})$ and then dried over anhydrous Na2SO4. Purification by flash chromatography on silica gel using a gradient of EtOAc/Hexane (0:100 to 60:40) afforded tert-butyl (3-(6-formyl-5-((2-methyl-5-(3-(trifluoromethyl)benzamido)phenyl) ethynyl)-7Hpyrrolo[2,3-d]pyrimidin-7-yl)propyl)carbamate as a pale brown solid $(65 \mathrm{mg}, 39 \%$; $\mathrm{Rf}=0.42$ in EtOAc:Hexane= 3:2). ${ }^{1} \mathrm{H}-\mathrm{NMR}\left(500 \mathrm{MHz}, \mathrm{CDCl}_{3}\right) \delta=10.26(\mathrm{~s}, 1 \mathrm{H}), 9.19(\mathrm{~s}, 1 \mathrm{H}), 9.02(\mathrm{~s}, 1 \mathrm{H})$, $8.72(\mathrm{~s}, 1 \mathrm{H}), 8.16(\mathrm{~s}, 1 \mathrm{H}), 8.10(\mathrm{~d}, J=7.8 \mathrm{~Hz}, 1 \mathrm{H}), 7.92(\mathrm{~s}, 1 \mathrm{H}), 7.76(\mathrm{~d}, J=7.7 \mathrm{~Hz}, 1 \mathrm{H}), 7.58(\mathrm{~d}$, $J=6.6 \mathrm{~Hz}, 1 \mathrm{H}), 7.21(\mathrm{~d}, J=8.3 \mathrm{~Hz}, 1 \mathrm{H}), 5.33(\mathrm{~s}, 1 \mathrm{H}), 4.68(\mathrm{t}, J=6.0 \mathrm{~Hz}, 2 \mathrm{H}), 3.12-2.95(\mathrm{~m}$, 2H), $2.48(\mathrm{~s}, 3 \mathrm{H}), 2.02-1.91(\mathrm{~m}, 2 \mathrm{H}), 1.43(\mathrm{~s}, 9 \mathrm{H})$. MS (ESI, m/z) calculated for $\mathrm{C}_{32} \mathrm{H}_{30} \mathrm{~F}_{3} \mathrm{~N}_{5} \mathrm{O}_{4}$ 605.2, $[\mathrm{M}+\mathrm{H}]^{+}$found 606.2.

(Z)- or $\quad(E)$-tert-butyl $\quad$ (3-(6-(3-amino-2-cyano-3-oxoprop-1-en-1-yl)-5-((2-methyl-5-(3(trifluoromethyl)benzamido)phenyl)ethynyl)-7H-pyrrolo[2,3- $d]$ pyrimidin-7-

yl)propyl)carbamate (15)

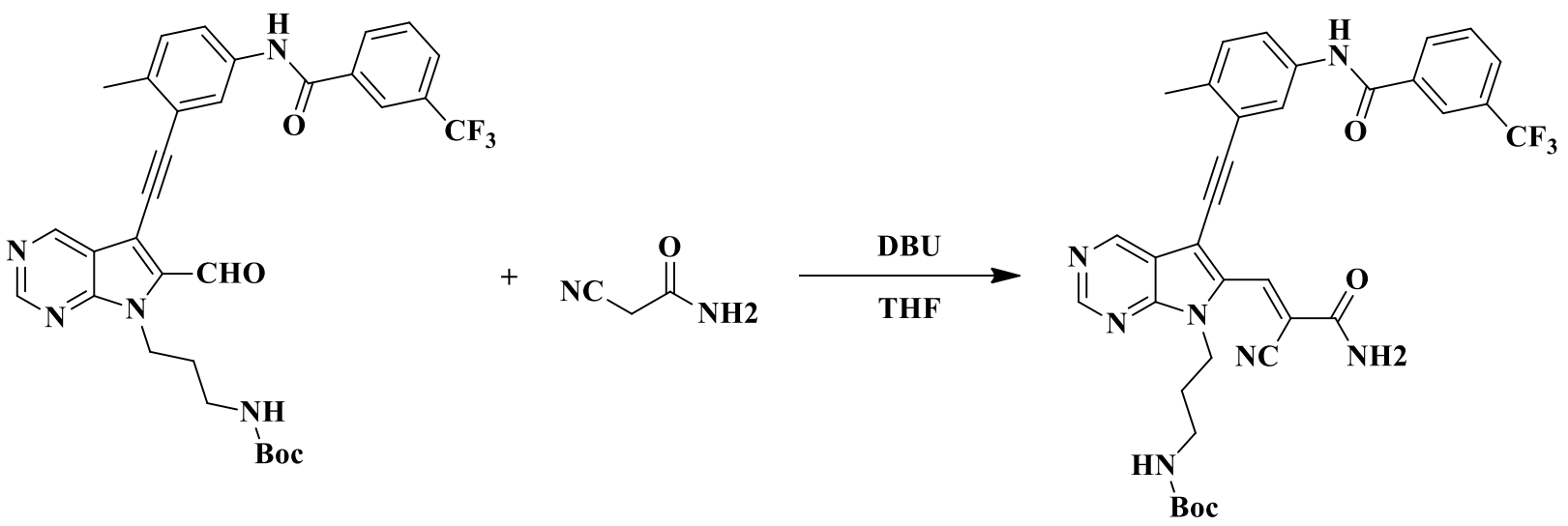

Tert-butyl (3-(6-formyl-5-((2-methyl-5-(3-(trifluoromethyl)benzamido)phenyl)ethynyl)-7Hpyrrolo[2,3-d]pyrimidin-7-yl)propyl)carbamate $\quad(33 \mathrm{mg}, \quad 0.054 \mathrm{mmol}, 1.0$ equiv. $)$ and 
cyanoacetamide (5.4 mg, $0.065 \mathrm{mmol}, 1.2$ equiv.) were dissolved in anhydrous THF (1.0 mL). 1,8Diazabicyclo[5.4.0]undec-7-ene (16 $\mathrm{mg}, 16 \mu \mathrm{L}, 0.11 \mathrm{mmol}, 2.0$ equiv.) was added and the resulting mixture was stirred at room temperature for 25 mins. The reaction was quenched with saturated $\mathrm{NH}_{4} \mathrm{Cl}$ solution and diluted with EtOAc $(20 \mathrm{~mL})$. The organic phase was washed with saturated $\mathrm{NaHCO}_{3}(4 \mathrm{~mL})$, brine $(4 \mathrm{~mL})$ and dried over anhydrous $\mathrm{Na}_{2} \mathrm{SO}_{4}$. Purification using preparative thin-layer chromatography developed with pure ethyl acetate afforded a mixture of (Z)- or (E)-tert-butyl (3-(6-(3-amino-2-cyano-3-oxoprop-1-en-1-yl)-5-((2-methyl-5-(3(trifluoromethyl)benzamido)phenyl)ethynyl)-7H-pyrrolo[2,3- $d$ ]pyrimidin-7-yl)propyl)carbamate as a bright yellow solid powder $\left(16 \mathrm{mg}, 44 \% ; \mathrm{R}_{\mathrm{f}}[(E)\right.$ and $(Z)$-isomers $\left.)\right]=0.48$ in $\mathrm{MeOH}: \mathrm{DCM}=$ 1:9). ${ }^{1} \mathrm{H}-\mathrm{NMR}(300 \mathrm{MHz}, \mathrm{MeOD}) \delta=9.18(\mathrm{~s}, 1 \mathrm{H}), 9.06(\mathrm{~s}, 1 \mathrm{H}), 8.96(\mathrm{~s}, 1 \mathrm{H}), 8.90(\mathrm{~s}, 1 \mathrm{H}), 8.42(\mathrm{~s}$, 2H), $8.28(\mathrm{~s}, 2 \mathrm{H}), 8.22(\mathrm{~d}, J=7.8 \mathrm{~Hz}, 2 \mathrm{H}), 8.07(\mathrm{~s}, 1 \mathrm{H}), 8.06(\mathrm{~s}, 1 \mathrm{H}), 7.92(\mathrm{~d}, J=6.7 \mathrm{~Hz}, 1 \mathrm{H})$, $7.89(\mathrm{~d}, J=6.7 \mathrm{~Hz}, 1 \mathrm{H}), 7.79-7.69(\mathrm{~m}, 2 \mathrm{H}), 7.68-7.59(\mathrm{~m}, 2 \mathrm{H}), 7.29(\mathrm{~d}, J=8.4 \mathrm{~Hz}, 2 \mathrm{H}), 5.12$ (s, 2H), $4.58-4.38(\mathrm{~m}, 4 \mathrm{H}), 3.13-2.98(\mathrm{~m}, 4 \mathrm{H}), 2.54(\mathrm{~s}, 6 \mathrm{H}), 2.11-1.91(\mathrm{~m}, 4 \mathrm{H}), 1.42(\mathrm{~s}, 18 \mathrm{H})$; MS (ESI, m/z) calculated for $\mathrm{C}_{35} \mathrm{H}_{32} \mathrm{~F}_{3} \mathrm{~N}_{7} \mathrm{O}_{4} 671.3,[\mathrm{M}+\mathrm{H}]^{+}$found 672.2 . 


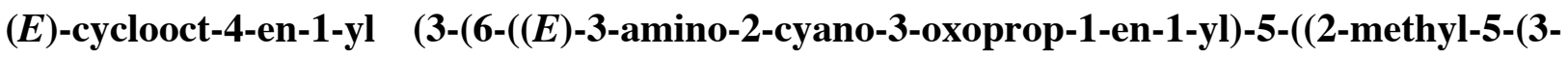
(trifluoromethyl)benzamido)phenyl)ethynyl)-7H-pyrrolo[2,3- $d]$ pyrimidin-7-

yl)propyl)carbamate or (E)-cyclooct-4-en-1-yl (3-(6-((Z)-3-amino-2-cyano-3-oxoprop-1-en1-yl)-5-((2-methyl-5-(3-(trifluoromethyl)benzamido)phenyl)ethynyl)-7H-pyrrolo[2,3d]pyrimidin-7-yl)propyl)carbamate (3-TCO)
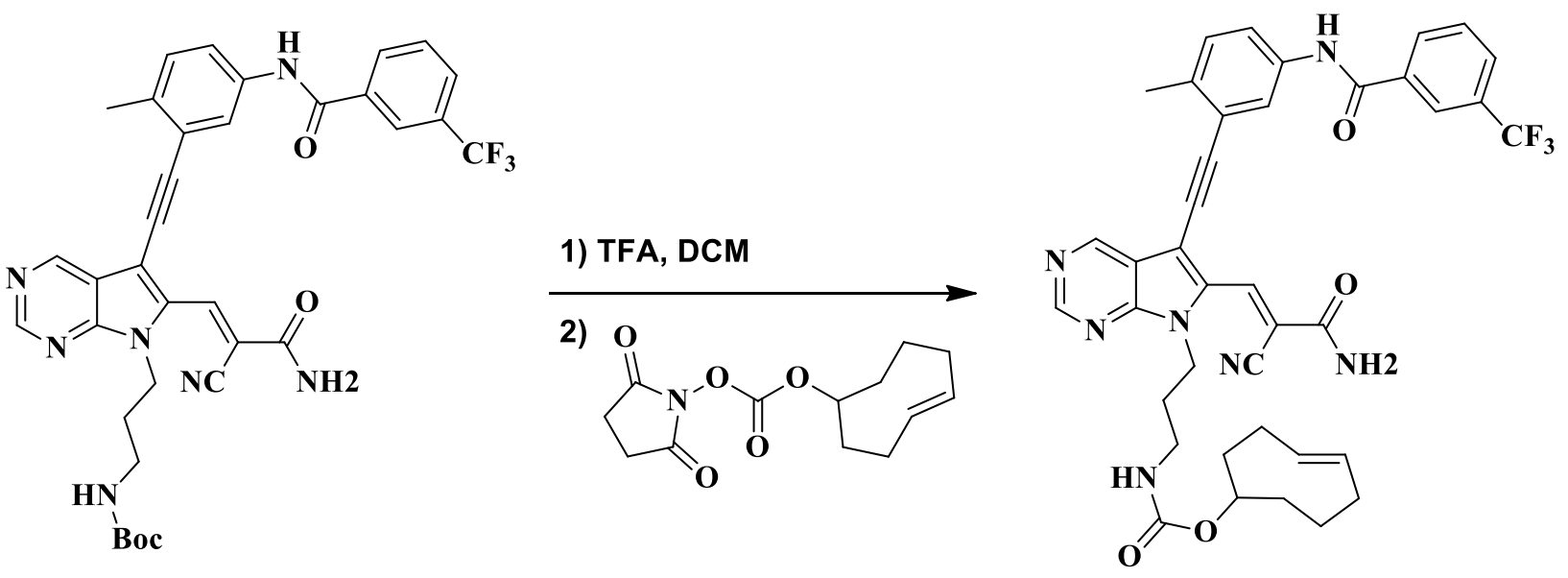

A mixture of (Z)- or (E)-tert-butyl (3-(6-(3-amino-2-cyano-3-oxoprop-1-en-1-yl)-5-((2-methyl-5(3-(trifluoromethyl)benzamido)phenyl)ethynyl)-7H-pyrrolo[2,3- $d]$ pyrimidin-7-

yl)propyl)carbamate (16 mg, $0.024 \mathrm{mmol}, 1.0$ equiv.) was dissolved in a mixture of trifluoacetic acid $(0.3 \mathrm{~mL})$ and dichloromethane $(0.7 \mathrm{~mL})$. The reaction solution was stirred at room temperature for 30 mins and the solvent was removed in vacuo. The solid residue was dissolved in anhydrous tetrahydrofuran $(1 \mathrm{~mL})$. To the above solution was added diisopropylethylamine (22 mg, $30 \mu \mathrm{L}, 0.17 \mathrm{mmol}, 5.0$ equiv.) and (E)-cyclooct-4-en-1-yl (2,5-dioxopyrrolidin-1-yl) carbonate (12 mg, $0.044 \mathrm{mmol}, 1.3$ equiv.) sequentially. The reaction was quenched with water (1 $\mathrm{mL}$ ) after maintaining at room temperature overnight. The resulting mixture was then diluted with ethyl acetate $(30 \mathrm{~mL})$. The organic phase washed with saturated $\mathrm{NaHCO}_{3}$ solution $(5 \mathrm{~mL})$, brine ( $5 \mathrm{~mL}$ ), dried over anhydrous $\mathrm{Na}_{2} \mathrm{SO}_{4}$. Purification using flash chromatography on silica gel with a EtOAc/Hexane gradient (0:100 to 100:0) afforded a mixture of (E)-cyclooct-4-en-1-yl (3-(6- 
((E)-3-amino-2-cyano-3-oxoprop-1-en-1-yl)-5-((2-methyl-5-(3-

(trifluoromethyl)benzamido)phenyl)ethynyl)-7H-pyrrolo[2,3- $d]$ pyrimidin-7-yl)propyl)carbamate or (E)-cyclooct-4-en-1-yl (3-(6-((Z)-3-amino-2-cyano-3-oxoprop-1-en-1-yl)-5-((2-methyl-5-(3(trifluoromethyl)benzamido)phenyl)ethynyl)-7H-pyrrolo[2,3- $d]$ pyrimidin-7-yl)propyl)carbamate as a bright yellow powder (10 mg, 59\% for 2 steps; $\operatorname{Rf}[(\mathrm{E})$ and $(\mathrm{Z})$-isomers $)]=0.50$ in pure EtOAc) ${ }^{1} \mathrm{H}-\mathrm{NMR}(500 \mathrm{MHz}, \mathrm{MeOD}) \delta=9.22(\mathrm{~s}, 1 \mathrm{H}), 9.10(\mathrm{~s}, 1 \mathrm{H}), 8.99(\mathrm{~s}, 1 \mathrm{H}), 8.94(\mathrm{~s}, 1 \mathrm{H}), 8.45$ (s, 2H), $8.32(\mathrm{~s}, 2 \mathrm{H}), 8.26(\mathrm{~d}, J=6.9 \mathrm{~Hz}, 2 \mathrm{H}), 8.13(\mathrm{~s}, 1 \mathrm{H}), 7.99(\mathrm{~s}, 1 \mathrm{H}), 7.94(\mathrm{~d}, J=6.0 \mathrm{~Hz}, 2 \mathrm{H}), 7.85-$ $7.73(\mathrm{~m}, 2 \mathrm{H}), 7.67(\mathrm{~d}, J=7.7 \mathrm{~Hz}, 2 \mathrm{H}), 7.34(\mathrm{~d}, J=8.0 \mathrm{~Hz}, 2 \mathrm{H}), 5.70-5.56(\mathrm{~m}, 2 \mathrm{H}), 5.55-5.44$ $(\mathrm{m}, 2 \mathrm{H}), 5.25(\mathrm{~s}, 4 \mathrm{H}), 5.16(\mathrm{~s}, 2 \mathrm{H}), 4.52(\mathrm{~s}, 4 \mathrm{H}), 4.30(\mathrm{~s}, 2 \mathrm{H}), 3.60-3.45(\mathrm{~m}, 2 \mathrm{H}), 3.29-3.20(\mathrm{~m}$, $2 \mathrm{H}), 3.17-3.10(\mathrm{~m}, 2 \mathrm{H}), 2.58(\mathrm{~s}, 6 \mathrm{H}), 2.43-0.72(\mathrm{~m}, 20 \mathrm{H})$; MS (ESI, m/z) calculated for $\mathrm{C}_{39} \mathrm{H}_{36} \mathrm{~F}_{3} \mathrm{~N}_{7} \mathrm{O}_{4} \mathrm{H}(\mathrm{M}+\mathrm{H})$ 724.2854, [M+H] $]^{+}$(HRMS) found 724.2842; HPLC purity: 98.2\% (UV detector wavelength: $254 \mathrm{~nm}$ ) 


\section{Expression and Purification of recombinant proteins}

\section{Recombinant Src expresssion}

N-terminal His-tagged Src V284C and Src WT (residue 87-536) were expressed and purified accordingly to the Supplementary Information of Mol. Cell 2019, 74, p. 393-408 for primary profiling data.

\section{Recombinant JNK2 expression}

His-TEV+LIC-JNK2 $\alpha 2$ WT and His-TEV+LIC-JNK2 $\alpha 2$ V40C/M108T were expressed in Escherichia coli BL21(DE3) cells in LB Miller broth. JNK2 $\alpha 2$ constructs were expressed in their inactive forms. Cells were grown to OD600 0.5 at $37{ }^{\circ} \mathrm{C}$. The temperature was reduced to $18{ }^{\circ} \mathrm{C}$ and cells were induced $30 \mathrm{~min}$ after temperature reduction with $0.4 \mathrm{M}$ isopropyl $\beta$-Dthiogalactoside. Expression occurred at $18{ }^{\circ} \mathrm{C}$ overnight. All purification steps were carried out at $4{ }^{\circ} \mathrm{C}$. Cells were pelleted in $1 \mathrm{~L}$ bottles via centrifugation at $6000 \mathrm{rpm}$. Cells were lysed with sonication in $2 \mathrm{~mL} / \mathrm{gram}$ pellet weight of wash/lysis buffer consisting of $50 \mathrm{mM}$ HEPES $(\mathrm{pH}=$ 7.5), $300 \mathrm{mM} \mathrm{NaCl}, 20 \mathrm{mM}$ imidazole, $10 \%$ glycerol and $1 \mathrm{mM}$ phenylmethylsulfonyl fluoride. The lysate was centrifuged at $10000 \mathrm{~g}$ for $20 \mathrm{~min}$ and the supernatant was allowed to batch bind for $60 \mathrm{~min}$ with $0.4 \mathrm{ml} / \mathrm{L}$ cell culture of Ni-NTA (Ni-nitrilotriacetate) resin. The resin was collected by centrifugation at $500 \mathrm{~g}$ for $5 \mathrm{~min}$ and washed with $20 \mathrm{~mL}$ of wash/lysis buffer per liter of culture. The wash step was repeated three times. The protein was eluted using $\sim 5 \mathrm{~mL}$ of elution buffer (50 mM HEPES (pH 7.5), 300 mM NaCl, 200 mM imidazole and 10\% glycerol) per liter of culture. Then, the eluate was dialyzed against $50 \mathrm{mM}$ HEPES ( $\mathrm{pH}=7.5), 200 \mathrm{mM} \mathrm{NaCl}, 5 \%$ glycerol and $1 \mathrm{mM}$ dithiothreitol (DTT) overnight. WT His-TEV+LIC-JNK2 $\alpha 2$ was treated with TEV during 
O.N. dialysis (2:25 wt:wt TEV:JNK2). The next day, the TEV cleaved WT JNK2 $\alpha 2$ was allowed to bind to $1 \mathrm{~mL}$ of Ni-NTA slurry for $1 \mathrm{hr}$ to remove the cleaved Hig tag and TEV. Cleaved JNK2 $\alpha 2$ was rinsed from the resin with wash/lysis buffer. Constructs were dialyzed two additional times. The aliquoted proteins were flash-frozen and stored at $-80{ }^{\circ} \mathrm{C}$. Protein concentrations were determined using in-gel BSA standards.

\section{Recombinant PAK1 expression}

PAK1 V286C/M346T (residues 250-547) was cloned into the bacterial expression plasmid pMCSG7 as a N-terminal His-SUMO-tagged construct. The construct was transformed in Escherichia coli BL21(DE3) cells and plated on ampicillin selective plates. A single colony was picked and grown in an overnight culture of $50 \mathrm{~mL}$ of LB broth containing ampicillin. An $1 \mathrm{~L}$ culture was inoculated with the starter culture, grown to an OD600 of 0.8. The protein expression was then induced with $0.2 \mathrm{M} \mathrm{IPTG}$ at $18{ }^{\circ} \mathrm{C}$. The next day, cells were harvested by centrifugation, lysed by sonication in lysis buffer (50 mM HEPES, $\mathrm{pH}=8.0,300 \mathrm{mM} \mathrm{NaCl}, 1 \mathrm{mM}$ PMSF, $0.1 \%$ Triton-X, $20 \mathrm{mM}$ imidazole), and centrifuged for 45 minutes at 10,000 rpm. Cleared lysates were incubated with $0.5 \mathrm{~mL}$ Ni-NTA resin (Thermo) for $1 \mathrm{~h}$. The supernatant was then discarded, and beads were washed with $20 \mathrm{~mL}$ lysis buffer before bound protein was eluted with lysis buffer containing $300 \mathrm{mM}$ imidazole. The eluted PAK1 V286C/M346T protein was dialyzed overnight at $4{ }^{\circ} \mathrm{C}$ in dialysis buffer ( $50 \mathrm{mM}$ Tris, $\mathrm{pH}=8.0,150 \mathrm{mM} \mathrm{NaCl}, 5 \%$ glycerol, $1 \mathrm{mM}$ DTT). PAK1 WT was purchased from SignalChem (cat. no. P02-10BG-05).

\section{Recombinant EphA2 expression}


The genes of N-terminal His-tagged EphA2 V627C and EphA2 WT (residue 590-876) were cloned into the bacterial expression plasmid pMCSG7. The construct was transformed in Escherichia coli BL21(DE3) cells and plated on ampicillin selective plates. A single colony was picked and grown in an overnight culture of $50 \mathrm{~mL}$ of LB broth containing ampicillin. The starting culture was then transferred to an one liter LB corth, grown to an OD600 of 1.2. Next, protein expression was induced with $0.2 \mathrm{M} \mathrm{IPTG}$ at $18{ }^{\circ} \mathrm{C}$. The next day, cells were harvested by centrifugation, lysed by sonication in lysis buffer (50 mM HEPES, $300 \mathrm{mM} \mathrm{NaCl}, 1 \mathrm{mM}$ PMSF, 0.1\% Triton-X, $20 \mathrm{mM}$ imidazole, $\mathrm{pH} 8.0$ ), and centrifuged for $45 \mathrm{~min}$ at 10,000 rpm. Cleared lysates were incubated with $0.5 \mathrm{~mL} \mathrm{Ni-NTA}$ resin (Thermo) for $1 \mathrm{~h}$. The supernatant was then discarded, and beads were washed with $20 \mathrm{~mL}$ lysis buffer before the bound protein was eluted with lysis buffer containing $300 \mathrm{mM}$ imidazole. The Eluted His-tagged recombinant EphA2 proteins were dialyzed overnight at $4{ }^{\circ} \mathrm{C}$ in dialysis buffer ( $50 \mathrm{mM}$ Tris, $\mathrm{pH}=8.0,150 \mathrm{mM} \mathrm{NaCl}, 5 \%$ glycerol, $1 \mathrm{mM}$ DTT). 
Protocol of activity assays to determine $K i$ values of 1,2,3 and TCO probes.

\section{Src activity assay}

Enzyme inhibition of Src V284C-variant or wild-type recombinant Src constructs (residue 87-536) was determined using a fluorogenic assay using a self-reporting Src kinase substrate EEEIYGE(DAP-Pyrene)-EA. 1, 2, 3, or TCO-conjugated probes (3-fold dilution, initial concentration of probe in reaction $=30 \mu \mathrm{M}$ ) were assayed in triplicate against $12.5 \mathrm{nM}$ of V284C-Src-3D or $7.5 \mathrm{nM}$ of WT Src-3D in kinase reaction buffer $\left([\mathrm{HEPES}]=75 \mathrm{mM}, \mathrm{pH}=8.0,\left[\mathrm{MgCl}_{2}\right]=15 \mathrm{mM},[\mathrm{EGTA}]=\right.$ $3.75 \mathrm{mM},[\mathrm{NaCl}]=150 \mathrm{mM},[\mathrm{BSA}]=0.2 \mathrm{mg} / \mathrm{mL}$ and $\left.\left[\mathrm{Na}_{3} \mathrm{VO}_{4}\right]=750 \mathrm{nM}\right)$. Src was incubated with inhibitors and ATP $(1 \mathrm{mM})$ for 30 mins in a Corning® 384 well microplate (Low flange; product number: CLS3573). Then $20 \mu \mathrm{M}$ of fluorogenic substrate solution in kinase reaction buffer was added and incubated for 120 mins. Fluorescent emission readouts were measured at wavelength of $405 \mathrm{~nm}$ when excited at $344 \mathrm{~nm}$ using a Perkin Elmer Envision Plate Reader. Percentage of inhibition was calculated and IC50 values were determined using One site - Fit $\log \mathrm{IC}_{50}$ model in GraphPad software Prism 7.

\section{PAK1 activity assay}

(Pakl) Pak1 WT and Pak1 V286C/M346T catalytic domains were catalytically active after expression in E. coli and purification. Pak1 WT (18 nM) and Pak1 V284C/M344T (35 nM) were incubated with 1, 2 or 3 (initial concentration $=10 \mu \mathrm{M}, 3$-fold serial dilutions, 10 data points), 4 $\mu \mathrm{M}$ cold ATP, and $0.007 \mu \mathrm{Ci} / \mu \mathrm{L} \gamma^{32} \mathrm{P}-\mathrm{ATP}$ for $30 \mathrm{~min}$ in assay buffer $(76 \mathrm{mM}$ HEPES, $\mathrm{pH}=7.5$, $5 \mathrm{mM} \mathrm{MgCl} 2,150 \mathrm{mM} \mathrm{NaCl}, 3.8 \mathrm{mM}$ EGTA, $\left.0.2 \mathrm{mg} / \mathrm{mL} \mathrm{BSA}, 150 \mathrm{uM} \mathrm{Na} \mathrm{VO}_{4}, 1 \mathrm{mM} \mathrm{BME}\right)$ then PAKtide (sequence:RRRLSFAEPG) (final concentration $=0.2 \mathrm{mg} / \mathrm{mL}$ ) was added. After 
incubating for $2-4 \mathrm{~h}, 4.6 \mu \mathrm{L}$ of the reaction mixture were spotted onto phosphocellulose. Membranes were washed with $0.05 \%$ phosphoric acid (3x 10 min wash) and air-dried, and the radioactivity was determined by phosphor-imaging with a GE Typhoon FLA 9000 phosphorscanner. Data was analyzed using GraphPad Prism software, and $\mathrm{IC}_{50}$ values were determined using "One-site fit $\log \mathrm{IC}_{50}$ " option. All assays were performed in triplicate.

\section{JNK2 activity assay}

Activated JNKs were prepared and activated using purified, activated MKK4 $\alpha .2 .5 \mu \mathrm{M}$ JNK2 was pre-activated with $150 \mathrm{nM} \mathrm{MKK} 4 \alpha$ for 1 hour at $\mathrm{RT}$ in $(50 \mathrm{mM}$ Tris/HCl, $\mathrm{pH}=7.5,0.01 \%(\mathrm{v} / \mathrm{v})$ Tween 20, $10 \mathrm{mM} \mathrm{MgCl}$, 2 mM DTT, 1 mM EGTA, $0.1 \mathrm{mg} / \mathrm{mL}$ BSA) with $400 \mu \mathrm{M}$ ATP.

To get an inhibitor dose response curve, an enzyme titration for JNK2 was first carried out in order to determine the linear working concentration of the enzyme. Inhibitors (initial concentration $=10$ $\mu \mathrm{M}$, 3-fold serial dilutions, 9 data points) were assayed in triplicate against kinases (JNK2 WT = $6.25 \mathrm{nM}$ and JNK2 V40C/M108T $=0.5 \mathrm{nM}$; in assay buffer $(50 \mathrm{mM}$ Tris $/ \mathrm{HCl}, \mathrm{pH}=7.5,0.01 \%$ (v/v) Tween 20, $10 \mathrm{mM} \mathrm{MgCl}_{2}, 0.1 \mathrm{mM}$ DTT, $1 \mathrm{mM}$ EGTA, $0.1 \mathrm{mg} / \mathrm{mL}$ BSA). JNK2 was incubated with inhibitors $4 \mu \mathrm{M}$ cold ATP, and $0.007 \mathrm{uCi} / \mathrm{uL}$ ATP-[ $\left.\gamma^{32} \mathrm{P}\right]$ for 30 mins in a 96-well plate. To initiate the reaction, myelin basic protein (MBP) was added, at a final concentration of $0.2 \mathrm{mg} / \mathrm{mL}$. The reaction mixture was incubated in a volume of $30.2 \mu \mathrm{L}$ per well, at room temperature, for 2 hours for JNK2 WT and $4 \mathrm{~h}$ for JNK2 V40C/M108T. To quench the reaction, $4.6 \mu l$ of the reaction mixture were spotted onto nitrocellulose, the membranes washed 3 times with $0.5 \%$ phosphoric acid and dried with acetone. Membranes were wrapped in plastic wrap and placed in a phosphor screen for autoradiography and exposed overnight. Phospho imaging was performed using a Typhoon FL 9000 instrument (GE Healthcare, Pittsburgh, PA). Data was 
analyzed using GraphPad Prism software, and $\mathrm{IC}_{50}$ values were determined using "One-site fit $\log \mathrm{IC}_{50} "$ option. All assays were performed in triplicate.

\section{$\operatorname{Eph} \mathrm{A} 2$ activity assay}

2.5 $\mathrm{nM}$ of recombinant EphA2 (residue 590-876) was incubated with inhibitors (initial concentration $=30 \mu \mathrm{M}, 3$-fold serial dilutions, 10 data points) for 30 minutes at room temperature in reaction buffer $\left([\mathrm{HEPES}]=50 \mathrm{mM}, \mathrm{pH}=7.5,\left[\mathrm{MgCl}_{2}\right]=10 \mathrm{mM}\right.$, [EGTA $]=1 \mathrm{mM}$, Tween-20= $0.1 \%,[\mathrm{DTT}]=1 \mathrm{mM}, \mathrm{BSA}=0.5 \mathrm{mg} / \mathrm{mL})$. The reaction mixture was then incubated with 0.0067 $\mathrm{mCi} / \mathrm{mL}$ of ${ }^{32} \gamma$-ATP and $2 \mathrm{mM}$ of cold ATP at room temperature for 30 minutes. EphA2 substrate Poly Glu:Tyr= 4:1 $(0.5 \mathrm{mg} / \mathrm{mL})$ was added and the reaction was incubated at room temperature for 4 hours. The reaction was terminated by spotting $4.6 \mu \mathrm{L}$ of the reaction mixture onto a phosphocellulose membrane. The membrane was washed with $0.05 \%$ phosphor 5 ic acid three times (10 mins each) and dried by air. The concentration of radioactively labeled EphA2 substrate was determined by phosphor-imaging with a GE Typhoon FLA 9000 phosphor-scanner. The image was processed and quantified with Image StudioLite software, and data were analyzed using GraphPad Prism, and $\mathrm{IC}_{50}$ values were determined using "One-site fit $\log \mathrm{IC}_{50}$ " option. All assays were performed in triplicate. 
SH3 domain pulldown assay: $10 \mu \mathrm{L}$ of a $50 \%$ slurry (by volume) of $\mathrm{O}^{6}$-benzylguanine-containing sepharose beads were washed with immobilization buffer $([$ Tris $]=50 \mathrm{mM}, \mathrm{pH}=7.5,[\mathrm{NaCl}]=100$ $\mathrm{mM},[\mathrm{DTT}]=1 \mathrm{mM},[\mathrm{BSA}]=0.2 \mathrm{mg} / \mathrm{mL})(10$ bed volumes for 3 times $)$. The washed beads were incubated with $50 \mu \mathrm{L}$ of SNAP-tagged-polyproline peptide (VSLARRPLPPLP) $(8 \mu \mathrm{M})$ on an endto-end rotator at ambient temperature for 1 hour. After immobilization of the polyproline peptide, the beads were washed with immobilization buffer (10-bed volumes for 3 times).

Meanwhile, a saturating amount of 1-TCO, 2-TCO, or 3-TCO $(3 \mu \mathrm{M}$ or $5 \mu \mathrm{M})$ was added to 50 $\mu \mathrm{L}$ of a solution in immobilization buffer containing $25 \mathrm{nM}$ of recombinant TAMRA-conjugated Src V284C. ${ }^{3,4}$ The solution was incubated at ambient temperature for 30 mins before being added to the polyproline peptide beads. After incubating at ambient temperature for 1 hour, the flowthrough was collected and the beads were washed with immobilization buffer (10-bed volumes for 3 times). The bead-bound Src was eluted by boiling the beads in $50 \mu \mathrm{L}$ of 1x SDS loading buffer at $95^{\circ} \mathrm{C}$ for 10 mins. Input (IN) and elution (EL) were processed using SDS-PAGE electrophoresis and imaged on GE-Typhoon FLA 9000. Intensities of rhodamine fluorescence for each band was quantified using ImageStudio ${ }^{\mathrm{TM}}$ Lite. Percentage of Src bound to the polyproline-containing beads= Intensity $(\mathrm{EL}) /[$ Intensity $(\mathrm{IN})+\operatorname{Intensity}(\mathrm{EL})] \times 100 \%$ was plotted using GraphPad software Prism 7. 


\section{Generation of Tetrazine-Beads}
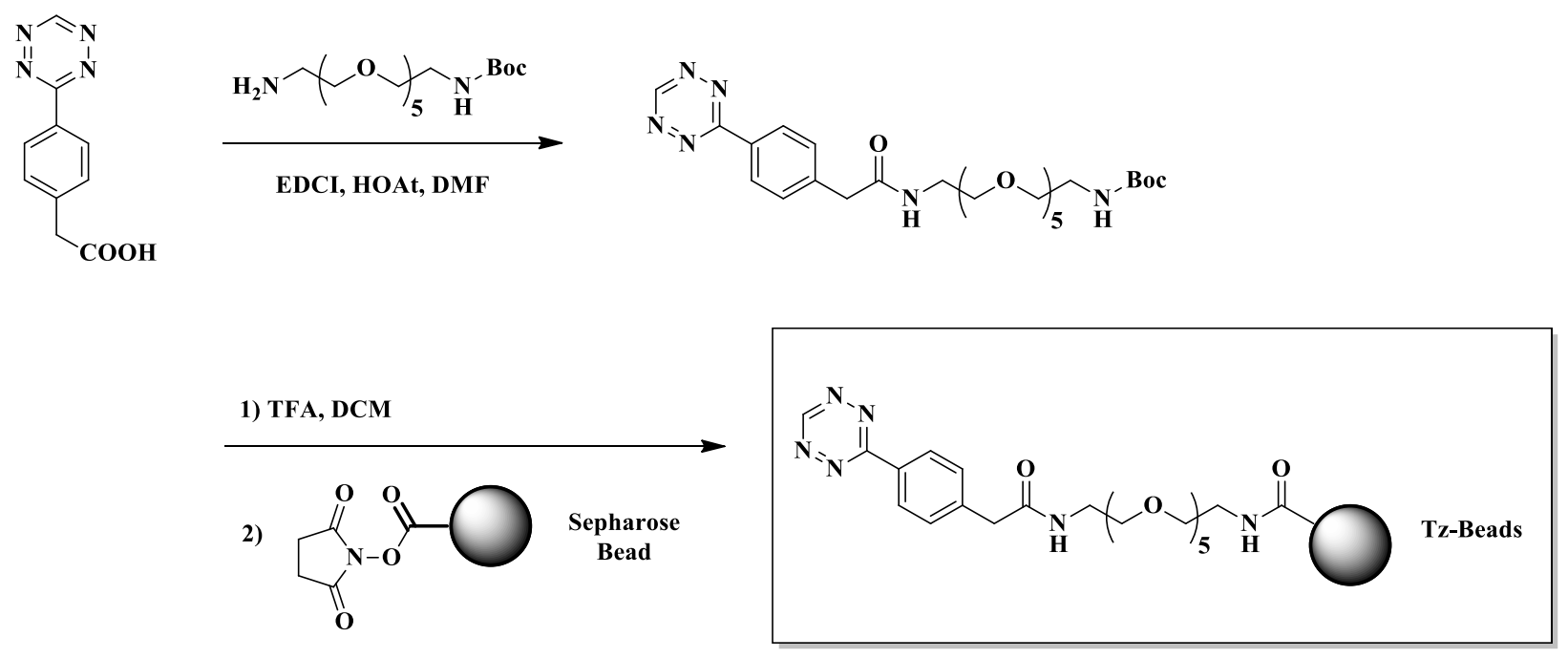

Tert-butyl (1-(4-(1,2,4,5-tetrazin-3-yl)phenyl)-2-oxo-6,9,12,15,18-pentaoxa-3-azaicosan-20yl)carbamate<smiles>O=C(O)Cc1ccc(-c2nncnn2)cc1</smiles>

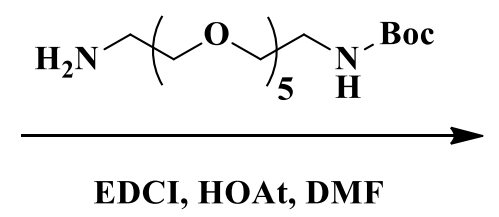

EDCI, HOAt, DMF<smiles>CC(COC(C)(C)CNC(=O)OC(C)(C)C)NC(=O)Cc1ccc(-c2nncnn2)cc1</smiles>

2-(4-(1,2,4,5-tetrazin-3-yl)phenyl)acetic acid (50 mg, $0.23 \mathrm{mmol}, 1.0$ equiv.), tert-butyl (17amino-3,6,9,12,15-pentaoxaheptadecyl)carbamate (114 mg, $0.30 \mathrm{mmol}, 1.3 \mathrm{~m}$ equiv.) and 1Hydroxy-7-azabenzotriazole ( $41 \mathrm{mg}, 0.30 \mathrm{mmol}, 1.3$ equiv.) were dissolved in anhydrous DMF (3 $\mathrm{mL}$ ). The solution was cooled to $0{ }^{\circ} \mathrm{C}$ and to it was added $N$-(3-Dimethylaminopropyl)- $N^{\prime}$ ethylcarbodiimide hydrochloride (58 $\mathrm{mg}, 0.30 \mathrm{mmol}, 1.3$ equiv.). The reaction was allowed to warm to room temperature overnight. The solvent was removed in vacuo and the solid residue was dissolved in ethyl acetate $(200 \mathrm{~mL})$. The organic phase was washed with saturated $\mathrm{KH}_{2} \mathrm{PO}_{4}$ solution $(30 \mathrm{~mL})$, saturated $\mathrm{NaHCO}_{3}$ solution $(30 \mathrm{~mL})$, brine $(30 \mathrm{~mL})$ and dried over anhydrous 
$\mathrm{Na}_{2} \mathrm{SO}_{4}$. Purification by flash chromatography on silica gel with a MeOH/EtOAc gradient $(0: 100$ to 20:80) afforded tert-butyl (1-(4-(1,2,4,5-tetrazin-3-yl)phenyl)-2-oxo-6,9,12,15,18-pentaoxa-3azaicosan-20-yl)carbamate as a bright pink solid (103 mg, 77\%). ${ }^{1} \mathrm{H}-\mathrm{NMR}\left(500 \mathrm{MHz}, \mathrm{CDCl}_{3}\right) \delta=$ $10.22(\mathrm{~s}, 1 \mathrm{H}), 8.59(\mathrm{~d}, J=7.9 \mathrm{~Hz}, 2 \mathrm{H}), 7.56(\mathrm{~d}, J=7.8 \mathrm{~Hz}, 2 \mathrm{H}), 6.69(\mathrm{~s}, 1 \mathrm{H}), 5.12(\mathrm{~s}, 1 \mathrm{H}), 3.68$ $(\mathrm{s}, 2 \mathrm{H}), 3.67-3.43(\mathrm{~m}, 22 \mathrm{H}), 3.32-3.26(\mathrm{~m}, 2 \mathrm{H}), 1.43(\mathrm{~s}, 9 \mathrm{H})$; MS (ESI, m/z) calculated for $\mathrm{C}_{27} \mathrm{H}_{42} \mathrm{~N}_{6} \mathrm{O}_{8}$ 578.3, $[\mathrm{M}+\mathrm{H}]^{+}$found 479.5 (cleavage product of Boc-protection group $\left.\mathrm{COOC}\left(\mathrm{CH}_{3}\right)_{3}\right)$; HPLC purity: $99.0 \%$ (UV detector wavelength: $254 \mathrm{~nm}$ )

\section{Immobilization of tetrazine}

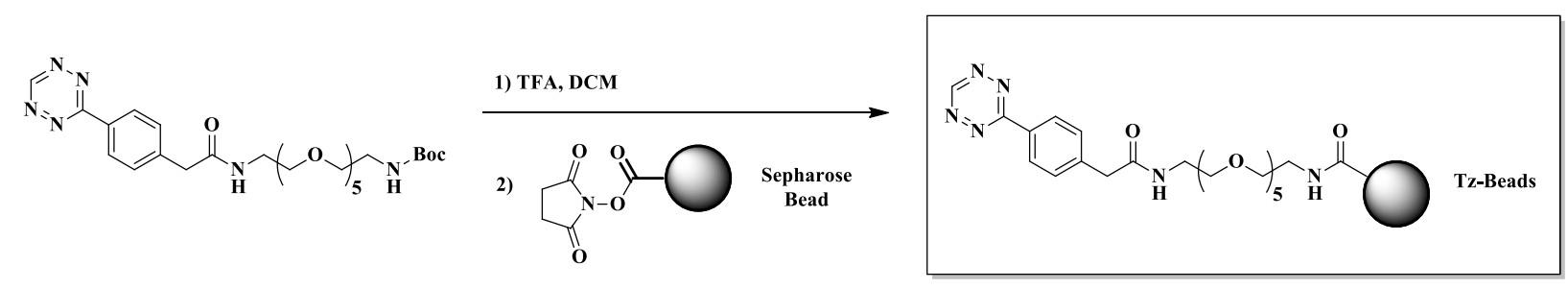

Tert-butyl (1-(4-(1,2,4,5-tetrazin-3-yl)phenyl)-2-oxo-6,9,12,15,18-pentaoxa-3-azaicosan-20yl)carbamate (22 mg, $0.037 \mathrm{mmol}, 1.0$ equiv.) was dissolved in a mixture of trifluoroacetic acid $(0.60 \mathrm{~mL})$ and dichloromethane $(1.4 \mathrm{~mL})$. The solution was maintained at room temperature for 30 mins. After reaction, the solvent was removed in vacuo and the residue was dissolved in a mixture of EtOH $(1.0 \mathrm{~mL})$ and DMF $(1.0 \mathrm{~mL})$ as coupling solution. Meanwhile, a 50\% slurry of NHS-activated Fast Flow sepharose beads $(1.0 \mathrm{~mL})$ were quickly washed three times with a mixed solvent consisting of EtOH and DMF (EtOH:DMF=1:1; use 1.0 mL for each wash) in 2-mL Pierce Centrifuge Columns (Thermo Scientific). The drained beads were resuspended in the coupling solution $(2 \mathrm{~mL})$ and to it was added triethylamine $(50 \mu \mathrm{L})$. The $\mathrm{pH}$ was of the supernatant was tested and adjusted to 7-8 with triethylamine if necessary. The reaction was agitated on a tube rotator at $4{ }^{\circ} \mathrm{C}$ overnight. On the second day, the beads were drained and washed three times with 
a mixed solvent consisting of EtOH and DMF (EtOH:DMF=1:1; use $1.0 \mathrm{~mL}$ for each wash), followed by three quick washes with an acid solution ([total $\mathrm{CH} 3 \mathrm{COOH} / \mathrm{NaOOCCH} 3]=0.1 \mathrm{M}$, $[\mathrm{NaCl}]=0.5 \mathrm{M}, \mathrm{pH}=4.0)$ and a basic solution $([$ Tris- $\mathrm{HCl}]=0.1 \mathrm{M},[\mathrm{NaCl}]=0.5 \mathrm{M}, \mathrm{pH}=8.0)(1 \mathrm{~mL}$ for each wash). The beads were then washed three times with $20 \% \mathrm{EtOH}$ in water ( $1 \mathrm{~mL}$ for each wash) and stored as $50 \%$ slurry in $20 \% \mathrm{EtOH}$ in water.

\section{Generation of sepharose beads for pre-clearing lysates}

$1 \mathrm{~mL}$ of $50 \%$ slurry of NHS-activated Fast Flow sepharose beads were quickly washed three times with a mixed solvent consisting of EtOH and DMF (EtOH:DMF=1:1; use $1.0 \mathrm{~mL}$ for each wash) in 2-mL Pierce Centrifuge Columns (Thermo Scientific). The washed beads were resuspended in a mixture of EtOH $(1 \mathrm{~mL})$ and $\mathrm{DMF}(1 \mathrm{~mL})$ containing $150 \mathrm{mM}$ ethanolamine. The reaction mixture was agitated on a tube rotator at $4^{\circ} \mathrm{C}$ overnight. The reaction was agitated on a tube rotator at $4{ }^{\circ} \mathrm{C}$ overnight. On the second day, the beads were drained and washed three times with a mixed solvent consisting of EtOH and DMF (EtOH:DMF=1:1; use $1.0 \mathrm{~mL}$ for each wash), followed by three quick washes with an acid solution ([total $\mathrm{CH} 3 \mathrm{COOH} / \mathrm{NaOOCCH} 3]=0.1 \mathrm{M},[\mathrm{NaCl}]=0.5$ $\mathrm{M}, \mathrm{pH}=4.0)$ and a basic solution ([Tris- $\mathrm{HCl}]=0.1 \mathrm{M},[\mathrm{NaCl}]=0.5 \mathrm{M}, \mathrm{pH}=8.0)(1 \mathrm{~mL}$ for each wash). The beads were then washed three times with $20 \% \mathrm{EtOH}$ in water ( $1 \mathrm{~mL}$ for each wash) and stored as $50 \%$ slurry in $20 \% \mathrm{EtOH}$ in water.

\section{Procedure for Enriching Probe-Bound Target in Cell Lysates}


Cell culture and generation of cell lysates: HEK293 cells were grown on $10-\mathrm{cm}$ plates in Dulbecco's Modified Eagle's Medium (DMEM) supplemented with 10\% (by volume) Fetal Bovine Serum (FBS) at $37{ }^{\circ} \mathrm{C}$ in a humidified incubator containing $5 \% \mathrm{CO}_{2}$ until $90 \%$ confluence. Cells were washed with ice-cold DPBS once and lysed with $500 \mu \mathrm{L}$ of ice-cold lysis buffer. Cell lysates were centrifuged at $17,000 \times \mathrm{g}$ for 10 minutes at $4{ }^{\circ} \mathrm{C}$ and were quantified using Pierce ${ }^{\mathrm{TM}} 660 \mathrm{~nm}$ Protein Assay Reagent and diluted to $1.5 \mathrm{mg} / \mathrm{mL}$ with ice-cold lysis buffer.

TCO probe treatment: Recombinant 3D construct of Src V284C or Src WT were added to the diluted HEK293 cell lysates to a final concentration of 100 nM. $1 \mu \mathrm{L}$ of 1-TCO, 2-TCO, or 3TCO solution (100× solution in DMSO) was added to $100 \mu \mathrm{L}$ of the lysate (DMSO $\%=1 \%$ in final solution). Lysates mixture was incubated with TCO probe for 30 minutes at $4{ }^{\circ} \mathrm{C}$ on an end-to-end rotator.

Capture probe-bound target using Tz-Beads: $5 \mu \mathrm{L}$ (volume of drained beads) of Tz-Beads were added to a $200 \mu \mathrm{L}$ tube and washed with ice-cold lysis buffer (three times; $100 \mu \mathrm{L}$ for each wash). $100 \mu \mathrm{L}$ of TCO probe-treated lysates mixture was added to the washed Tz-Beads and was gently incubated on an end-to-end rotator for 30 mins (or the indicated amount of time in Figure 3G) at $4{ }^{\circ} \mathrm{C}$. After incubation with Tz-Beads, the flow-through was quickly removed by aspiration. The beads were then washed for three times with ice-cold Washing Buffer 1 (100 $\mu \mathrm{L}$ for each wash) by gently inverting the tube for four times. The probe-bound target should be bound to Tz-Beads by the end of this step.

Elute enriched probe-bound target and Western blot analysis: The sepharose matrix was incubated with $100 \mu \mathrm{L}$ of Lysis Buffer containing $2 \%$ SDS (by weight) for 20 mins at $25{ }^{\circ} \mathrm{C}$ with agitation. The eluted proteins were separated using SDS-PAGE gel electrophoresis, transferred 
onto nitrocellulose membranes and immunoblotted with corresponding primary antibodies. The nitrocellulose membranes were imaged on LI-COR Odyssey IR imager. 


\section{Enrichment of probe-bound Src kinase complex from live mammalian cell}

\section{Cell culture and TCO probe treatment:}

(Condition a: Serum starvation) Hela cells that stably overexpressed full-length wild-type Src and V284C variant of Src were grown on 10-cm plates in Dulbecco's Modified Eagle's Medium (DMEM) supplemented with $10 \%$ (by volume) Fetal Bovine Serum (FBS) at $37^{\circ} \mathrm{C}$ in a humidified incubator containing 5\% $\mathrm{CO}_{2}$ until $90 \%$ confluence, and was serum-starved for 24 hours prior to the assay. Expression of Src WT and Src V284C was induced by addition of Doxycycline (1 $\mu \mathrm{g} / \mathrm{mL}$ ) 24 hours prior to the assay. Click probes were added to the cells by replacing medium with fresh DMEM $(5 \mathrm{~mL})$ containing 1-TCO $(5 \mu \mathrm{M}$; DMSO $\%=0.1 \%$ in final medium $)$ or 2-TCO $(2.5$ $\mu \mathrm{M} ; \mathrm{DMSO} \%=0.1 \%$ in final medium). Cells were incubated with 1-TCO or 2-TCO for 30 minutes at $37{ }^{\circ} \mathrm{C}$ in the incubator and the medium was removed immediately after incubation. The cells were quickly washed with ice-cold Dulbecco's Phosphate-Buffered Saline (DPBS) (5 mL, one time) and immediately lysed with ice-cold Lysis Buffer $(500 \mu \mathrm{L})$. Cells were scrapped into pre-chilled 1.5-mL Eppendorf tubes and were centrifuged at $17,000 \times \mathrm{g}$ for 20 minutes at $4{ }^{\circ} \mathrm{C}$. The supernatant of lysates was collected, and protein concentration was measured using Pierce $660 \mathrm{~nm}$ Protein Assay. The protein concentration of lysates was diluted to $1.5 \mathrm{mg} / \mathrm{mL}$ with icecold lysis buffer.

(Condition b: Complete medium (DMEM)) Hela cells that stably overexpressed full-length wild-type Src and V284C variant of Src were grown on 10-cm plates in Dulbecco's Modified Eagle's Medium (DMEM) supplemented with 10\% (by volume) Fetal Bovine Serum (FBS) at 37 ${ }^{\circ} \mathrm{C}$ in a humidified incubator containing $5 \% \mathrm{CO}_{2}$ until $90 \%$ confluence. Expression of Src WT and Src V284C was induced by addition of Doxycycline $(1 \mu \mathrm{g} / \mathrm{mL}) 24$ hours prior to the assay. Click probes were added to the cells by replacing medium with fresh DMEM $(5 \mathrm{~mL})$ containing $10 \%$ 
FBS and 1-TCO $(5 \mu \mathrm{M}$; DMSO $\%=0.1 \%$ in final medium $)$ or 2-TCO $(2.5 \mu \mathrm{M}$; DMSO $\%=0.1 \%$ in final medium). Cells were incubated with 1-TCO or 2-TCO for 30 minutes at $37{ }^{\circ} \mathrm{C}$ in the incubator and the medium was removed immediately after incubation. The cells were quickly washed with ice-cold Dulbecco's Phosphate-Buffered Saline (DPBS) (5 mL, one time) and immediately lysed with ice-cold Lysis Buffer $(500 \mu \mathrm{L})$. Cells were scrapped into pre-chilled 1.5$\mathrm{mL}$ Eppendorf tubes and were centrifuged at $17,000 \times \mathrm{g}$ for 20 minutes at $4{ }^{\circ} \mathrm{C}$. The supernatant of lysates was collected, and protein concentration was measured using Pierce $660 \mathrm{~nm}$ Protein Assay. The protein concentration of lysates was diluted to $1.5 \mathrm{mg} / \mathrm{mL}$ with ice-cold lysis buffer.

(Condition $c$ : Growth factor) Hela cells that stably overexpressed full-length wild-type Src and V284C variant of Src were grown on 10-cm plates in Dulbecco's Modified Eagle's Medium (DMEM) supplemented with $10 \%$ (by volume) Fetal Bovine Serum (FBS) at $37^{\circ} \mathrm{C}$ in a humidified incubator containing 5\% $\mathrm{CO}_{2}$ until $90 \%$ confluence, and was serum-starved for 24 hours prior to the assay. Expression of Src WT and Src V284C was induced by addition of Doxycycline (1 $\mu \mathrm{g} / \mathrm{mL}) 24$ hours prior to the assay. Click probes were added to the cells by replacing medium with fresh DMEM $(5 \mathrm{~mL})$ containing 10\% FBS and 1-TCO $(5 \mu \mathrm{M}$; DMSO $\%=0.1 \%$ in final medium $)$ or 2-TCO $(2.5 \mu \mathrm{M} ; \mathrm{DMSO} \%=0.1 \%$ in final medium $)$. Cells were incubated with 1-TCO or 2TCO for 30 minutes at $37{ }^{\circ} \mathrm{C}$ in the incubator. The medium was then immediately replaced with $5 \mathrm{~mL}$ of DMEM containing 10\% FBS and EGF (100 ng/mL). After 10 minutes, the medium was removed immediately. The cells were quickly washed with ice-cold Dulbecco's PhosphateBuffered Saline (DPBS) (5 mL, one time) and immediately lysed with ice-cold Lysis Buffer (500 $\mu \mathrm{L})$. Cells were scrapped into pre-chilled 1.5-mL Eppendorf tubes and were centrifuged at 17,000 $\times \mathrm{g}$ for 20 minutes at $4{ }^{\circ} \mathrm{C}$. The supernatant of lysates was collected, and protein concentration 
was measured using Pierce $660 \mathrm{~nm}$ Protein Assay. The protein concentration of lysates was diluted to $1.5 \mathrm{mg} / \mathrm{mL}$ with ice-cold lysis buffer.

(Condition $\boldsymbol{d}$ : Fibronectin) 10-cm petri dish plates (Petri Dishes with Clear Lid. Thermo Fisher, catalog No. FB0875712) were treated with $5 \mathrm{~mL}$ of $10 \mu \mathrm{g} / \mathrm{mL}$ of Fibronectin solution in DPBS for 1 hour at $37^{\circ} \mathrm{C}$. The plates were washed with DPBS once before usage. Hela cells that stably overexpressed full-length wild-type $\mathrm{Src}$ and V284C variant of Src were grown on 10-cm fibronectin-coated plates in Dulbecco's Modified Eagle's Medium (DMEM) supplemented with $10 \%$ (by volume) Fetal Bovine Serum (FBS) at $37{ }^{\circ} \mathrm{C}$ in a humidified incubator containing $5 \%$ $\mathrm{CO}_{2}$ until $90 \%$ confluence. Expression of Src WT and Src V284C was induced by addition of Doxycycline $(1 \mu \mathrm{g} / \mathrm{mL}) 24$ hours prior to the assay. Click probes were added to the cells by replacing medium with fresh DMEM $(5 \mathrm{~mL})$ containing $10 \%$ FBS and 1-TCO $(5 \mu \mathrm{M}$; DMSO $\%=$ $0.1 \%$ in final medium) or 2-TCO $(2.5 \mu \mathrm{M}$; $\mathrm{DMSO} \%=0.1 \%$ in final medium $)$. Cells were incubated with click probes for 30 minutes at $37^{\circ} \mathrm{C}$ in the incubator. The cells were quickly washed with ice-cold Dulbecco's Phosphate-Buffered Saline (DPBS) (5 mL, one time) and immediately lysed with ice-cold Lysis Buffer (500 $\mu \mathrm{L})$. Cells were scrapped into pre-chilled 1.5-mL Eppendorf tubes and were centrifuged at $17,000 \times \mathrm{g}$ for 20 minutes at $4{ }^{\circ} \mathrm{C}$. The supernatant of lysates was collected, and protein concentration was measured using Pierce $660 \mathrm{~nm}$ Protein Assay. The protein concentration of lysates was diluted to $1.5 \mathrm{mg} / \mathrm{mL}$ with ice-cold lysis buffer.

Capture probe-bound kinase complexes using Tz-Beads: For each co-clickable precipitation experiment, $15 \mu \mathrm{L}$ (volume of drained beads) of Tz-Beads were added to a 1.5-mL Eppendorf tube and were washed with ice-cold lysis buffer (three times; $400 \mu \mathrm{L}$ for each wash). $750 \mu \mathrm{g}$ of protein lysates $(1.5 \mathrm{mg} / \mathrm{mL})$ was added to Tz-Beads $(500 \mu \mathrm{L})$ and was gently incubated on an end-to-end rotator for 30 mins at $4{ }^{\circ} \mathrm{C}$. To reduce background contaminants, lysates were pre-cleared by 
incubating with $15 \mu \mathrm{L}$ (volume of drained beads) of sepharose beads coupled to ethanolamine for 1 hour at $4{ }^{\circ} \mathrm{C}$ prior to incubation with Tz-beads. After incubation with Tz-Beads, the flow-through was quickly removed by aspiration. The beads were then washed with ice-cold Washing Buffer 1 by gently inverting the tube for four times (three times; $400 \mu \mathrm{L}$ for each wash), followed by washing with Washing Buffer 2 by gently inverting the tube for four times (three times; $400 \mu \mathrm{L}$ for each wash). The bait protein and its interactors should be enriched and bound to the Tz-Beads by the end of this step.

Characterization of kinase complex using Western-blot analysis: To detect the components of proteins enriched by Tz-Beads using Western-blot analysis, the beads were incubated with Lysis Buffer containing $2 \%$ SDS (by weight) for 20 mins (three times; $40 \mu \mathrm{L}$ for each elution) at $25{ }^{\circ} \mathrm{C}$ with frequent agitation. The elutions were collected. The eluted proteins were processed using SDS-PAGE gel electrophoresis, transferred onto nitrocellulose membranes and immunoblotted with corresponding primary antibodies. The western-blots were imaged on LI-COR Odyssey IR imager.

On-bead tryptic digestion of kinase complexes for tandem mass spectrometry analysis: To detect enriched bait and interactors using LC/MS, the washed beads were resuspended in $50 \mu \mathrm{L}$ of Denaturing Buffer and were boiled at $95{ }^{\circ} \mathrm{C}$ for 5 minutes. After cooling down, $100 \mathrm{mM}$ TEAB $(50 \mu \mathrm{L})$ was added. The $\mathrm{pH}$ value was adjusted to 8 to 9 by adding $\mathrm{NaOH}(1 \mathrm{M})$. Lys-c Lysyl Endopeptidase $(1 \mu \mathrm{g})$ was then added and incubated with the beads at $37^{\circ} \mathrm{C}$ for 2 hours in a thermal mixer agitating at 1,400 rpm. After incubation, the suspension was diluted with $100 \mathrm{mM}$ TEAB $(100 \mu \mathrm{L})$ and MS Grade Trypsin $(1 \mu \mathrm{g})$ was added. $\mathrm{pH}$ was adjusted to 8 to 9 . The beads were incubated with Trypsin overnight at $37{ }^{\circ} \mathrm{C}$ in a thermal mixer agitating at $1,400 \mathrm{rpm}$. After incubation, Buffer A ( $200 \mu \mathrm{L}$ ) was added to the beads and trypsin was quenched by adjusting the 
$\mathrm{pH}$ to 2 to 3 using formic acid. The resulting supernatant was desalted on C18 StageTip and washed once by buffer A $(50 \mu \mathrm{L})$. The tryptic peptides were eluted off the C18 StageTip using buffer B $(50 \mu \mathrm{L})$. The eluted solution was dried in speed-vac and then reconstituted with buffer A (20 $\mu \mathrm{L})$ for LC/MS analysis. 


\section{Proteomic Data Acquisition, Process and Visualization of Target Interactome.}

Proteomic Data Acquisition: Peptides were separated on a nanoAcquity UPLC instrument with $12 \mathrm{~cm}$ long fused silica capillary column (Polymicro Technologies Flexible Fused Silica Capillary Tubing, Inner Diameter $75 \mu \mathrm{m}$, Outer Diameter $375 \mu \mathrm{m}$, cat. no. 1068150019) made in-house with a laser puller and packed with $5 \mu \mathrm{m} 120$ Å reversed phase C18 beads (ReproSil-Pur $120 \mathrm{C} 18-\mathrm{AQ}$, Dr. Maisch GmbH HPLC, cat. no. r15.aq.). The LC gradient was 90 min long with 10-35\% B at $200 \mathrm{~nL} / \mathrm{min}$. LC solvent A was $0.1 \%$ acetic acid and LC solvent B was $0.1 \%$ acetic acid, $99.9 \%$ acetonitrile. MS data were collected with a Thermo Scientific Orbitrap Fusion Tribrid mass spectrometer.

Proteomic Data Process: Raw data were analyzed using MaxQuant and search engine Andromeda using the following default settings: Multiplicity was set to 1; Digestion was set to trypsin/P; Variable modifications were set to Oxidation (M) and Acetyl (Protein N-term); LFQ minimal ratio count was set to 2 ; $\mathrm{LFQ}$ minimal number of neighbors set to 3 ; $\mathrm{LFQ}$ average number of neighbors set to 6; MS/MS spectra were searched against the UniProt human database (updated July 22nd, 2015); False discovery rate (FDR) was set to 0.01; First search and MS/MS search mass tolerance was set to $10 \mathrm{ppm}$; The data in the Protein Groups result table was processed with Perseus software. MaxQuant intensity values of identified proteins were used for all downstream analysis. We only consider proteins identified if MaxQuant was able to compute corresponding protein intensity values. Identified proteins that were classified as "only identified by site", "reverse", or "contaminant" were filtered and removed from the table. Proteins with 1 or fewer peptide counts were filtered and removed. All intensity values were transformed based on $\log 2$ algorithm. Missing protein intensity values for identified proteins were imputed using Perseus by sampling from a distribution downshifted by 1.3 and having a width of $0.2^{5,6}$. Imputed values are reported in the 
SI-Excel file 1. P-value was calculated using two-tailed, two-sample T-test. Enrichment scores ( $\log _{2}[$ intensity (Src V284C-expressing)/intensity (Src WT-expressing)]) were calculated using the relative intensity values from Src V284C- and WT-expressing cells with confidence values ($\log _{10}(\mathrm{P}$-value $\left.)\right)$ for each identified protein. We defined significant interactors as protein hits with an enrichment score $\geq 1.0$ and a confidence value $\geq 1.4$.

Visualization of Target Interactome: The list of gene names for significant Src interactors were uploaded to STRING: functional protein association networks database (version 10.5). The organism was set to Homo sapiens. The meaning of network edges was set to the confidence of the interaction, where the line thickness indicates the strength of data support as calculated by the STRING network database. ${ }^{1}$ The edge confidence was indicated in Figure 4E. The minimum required interaction score is 0.400 . The colored nodes represent query proteins. Each node represents all the proteins produced by a single, protein-coding gene locus. The splice isoforms or post-translational modifications are collapsed into the same node. 


\section{Clickable Proximity Ligation Assays (PLA).}

Cell seeding: (for Figure 6B and Figure 6C) $40 \mu \mathrm{L}$ of $0.8 \times 10^{5}$ cells $/ \mathrm{mL}$ of Hela cells expressing Src-V284C or Src-WT were plated in Grace Bio-labs CultureWell chambered coverglass ( 8 wells, well diam. $\times$ thickness $6 \mathrm{~mm} \times 1 \mathrm{~mm}$, well volume 15-30 $\mu$ L; Sigma-Aldrich; Catalog number: GBL103380) in DMEM supplemented with 10\% FBS. The cells were then incubated until reaching 30-40\% confluency in a humidified incubator at $37{ }^{\circ} \mathrm{C}$ containing $5 \% \mathrm{CO}_{2}$; (for Figure 6D) Prior to cell seeding, chambered coverglass was incubated with $40 \mu \mathrm{L}$ of $1 \mathrm{mg} / \mathrm{mL}$ Fibronectin solution for 30 minutes at $37{ }^{\circ} \mathrm{C}$ for 30 mins and then rinsed with DPBS once. $40 \mu \mathrm{L}$ of $0.8 \times 10^{5}$ cells/mL of Hela cells expressing Src-V284C or Src-WT were plated in Grace Bio-labs CultureWell chambered coverglass $(8$ wells, well diam. $\times$ thickness $6 \mathrm{~mm} \times 1 \mathrm{~mm}$, well volume 15-30 $\mu$ L; Sigma-Aldrich; Catalog number: GBL103380) in DMEM supplemented with 10\% FBS. The cells were then incubated until reaching $30-40 \%$ confluency in a humidified incubator at $37^{\circ} \mathrm{C}$ containing $5 \% \mathrm{CO}_{2}$

Probe treatment: Cells were treated with $50 \mu \mathrm{L}$ of 1-TCO $(5 \mu \mathrm{M}), 2-\mathrm{TCO}(2.5 \mu \mathrm{M})$ or 3-TCO $(10 \mu \mathrm{M})$ in DMEM supplemented with 10\% FBS for 30 minutes.

Cell fixation and permeabilization: After incubation, the cells were gently washed with warm DPBS (40 $\mu \mathrm{L}$ ) once and fixed with $40 \mu \mathrm{L}$ of $4 \%$ paraformaldehyde for 15 minutes at room temperature. To quench the remaining para-formaldehyde, cells were then incubated with $40 \mu \mathrm{L}$ of $125 \mathrm{mM}$ of Glycine in DPBS for 5 mins. After washing with DBPS once, the cells were permeabilized using DPBS containing $0.75 \%$ Tween-20 and 0.75\% Igepal CA-630 for 60 minutes at room temperature in a coplin jar on a shaker. The cells were then washed with DBPS once for 5 minutes at room temperature. 
Click chemistry: Cells were treated with $40 \mu \mathrm{L}$ of $500 \mathrm{nM}$ Tetrazine-conjugated OregonGreen 488 in DPBS for 10 minutes at room temperature. After the click chemistry reaction, the reaction solution was removed from chambered coverglass and the cells were washed with DPBS for 5 minutes. The washing step was repeated for six times in total.

Blocking and treatment with primary antibodies: Cells were blocked with blocking buffer (Sigma Duolink buffer) at $37^{\circ} \mathrm{C}$ for 1 hour. After blocking, blocking buffer was aspirated and cells were then treated with $40 \mu \mathrm{L}$ of a solution consisted of primary antibodies in Antibody Dilution Buffer (Sigma Duolink) for OregonGreen488 (anti-fluorescein/OregonGreen goat IgG fraction; Life technologies; Catalog number: A11095) and Src(anti-Src (36D10) Rabbit mAb; Cell Signaling Technology; Catalog \#: 2109), EEA1 (anti-EEA1 (C45B10) Rabbit mAb; Cell Signaling Technology; Catalog \#: 3288) or FAK (anti-FAK (D5O7U) XP Rabbit mAb; Cell Signaling Technology; Catalog \#: 71433) at $4{ }^{\circ} \mathrm{C}$ overnight. After incubation, the cells were washed with Wash Buffer A (Sigma Duolink) for 5 minutes twice in a coplin jar on a shaker.

Treatment with secondary antibodies and proximity ligation: Cells were then treated with 40 $\mu \mathrm{L}$ of a solution containing Duolink in situ rabbit PLUS probes and Duolink in situ goat MINUS probes in Antibody Dilution Buffer at $37{ }^{\circ} \mathrm{C}$ for 1 hour. After incubation, the cells were washed with Wash Buffer A for 5 minutes for three times. $40 \mu \mathrm{L}$ of Ligation solution containing DNA ligase in ligation buffer was added to the cells. Cells were incubated with ligase for 30 minutes at $37^{\circ} \mathrm{C}$. After DNA ligation, cells were washed with Wash Buffer A for 5 minutes twice. For rolling cycle amplification, cells were treated with $40 \mu \mathrm{L}$ of amplification buffer consisted of Polymerase and polymerase buffer (Sigma Duolink In Situ Detection Reagents Orange; Catalog \#: DUO92007) for exactly 100 minutes at $37^{\circ} \mathrm{C}$. After incubation, the cells were washed with Wash Buffer B for 10 minutes twice at room temperature in a coplin jar on a shaker. The excess wash 
solution was removed. To stain nuclei, one drop of the Duolink In Situ mounting media containing DAPI was added to each well and the coverglass was mounted to a glass coverslip.

Imaging, data acquisition, and analysis: Cells were imaged using a Leica SP8X Confocal microscope equipped with a $63 \mathrm{X}$ objective (oil objective). Nuclei were imaged using laser excitation at $405 \mathrm{~nm}$ with 5\% laser power. PLA puncta were imaged using laser excitation at 554 $\mathrm{nm}$ with $10 \%$ laser power. The number of PLA punctae was quantified using ImageJ software as following: Firstly, images were smoothened. Secondly, threshold was manually selected to discriminate PLA puncta from background fluorescence. Once selected, the threshold was applied uniformly to all images in the same sample set. The number of PLA puncta in each image was quantified using "Analyze Particles" function with the particle size of " $0.75 \mu \mathrm{m}^{2}-200 \mu \mathrm{m}^{2}$ ". The number of cells in each image was quantified using the number of nucleus. As an assay readout, the number of PLA puncta per cell was calculated. All data were visualized in violin plots using GraphPad Prism software. 


\section{Reference:}

1. Szklarczyk, D.; Franceschini, A.; Wyder, S.; Forslund, K.; Heller, D.; Huerta-Cepas, J.; Simonovic, M.; Roth, A.; Santos, A.; Tsafou, K. P.; Kuhn, M.; Bork, P.; Jensen, L. J.; von Mering, C., STRING v10: protein-protein interaction networks, integrated over the tree of life. Nucleic Acids Res 2015, 43 (Database issue), D447-52.

2. Szklarczyk, D.; Morris, J. H.; Cook, H.; Kuhn, M.; Wyder, S.; Simonovic, M.; Santos, A.; Doncheva, N. T.; Roth, A.; Bork, P.; Jensen, L. J.; von Mering, C., The STRING database in 2017: quality-controlled protein-protein association networks, made broadly accessible. Nucleic Acids Res 2017, 45 (D1), D362-D368.

3. Lombard, C. K.; Davis, A. L.; Inukai, T.; Maly, D. J., Allosteric Modulation of JNK Docking Site Interactions with ATP-Competitive Inhibitors. Biochemistry 2018, 57 (40), 58975909.

4. Chakraborty, S.; Inukai, T.; Fang, L.; Golkowski, M.; Maly, D. J., Targeting Dynamic ATP-Binding Site Features Allows Discrimination between Highly Homologous Protein Kinases. ACS Chemical Biology 2019, 14 (6), 1249-1259.

5. Tyanova, S.; Temu, T.; Sinitcyn, P.; Carlson, A.; Hein, M. Y.; Geiger, T.; Mann, M.; Cox, J., The Perseus computational platform for comprehensive analysis of (prote)omics data. Nat Methods 2016, 13 (9), 731-40.

6. Golkowski, M.; Vidadala, R. S.; Lombard, C. K.; Suh, H. W.; Maly, D. J.; Ong, S. E., Kinobead and Single-Shot LC-MS Profiling Identifies Selective PKD Inhibitors. J Proteome Res 2017, $16(3), 1216-1227$. 
HRMS data:

(E)-cyclooct-4-en-1-yl (3-(4-amino-5-(4-chlorophenyl)-6-((E)-2-cyano-3-(isopropylamino)-3oxoprop-1-en-1-yl)-7H-pyrrolo[2,3- $d]$ pyrimidin-7-yl)propyl)carbamate or (E)-cyclooct-4en-1-yl (3-(4-amino-5-(4-chlorophenyl)-6-((Z)-2-cyano-3-(isopropylamino)-3-oxoprop-1-en1-yl)-7H-pyrrolo[2,3-d]pyrimidin-7-yl)propyl)carbamate (1-TCO)

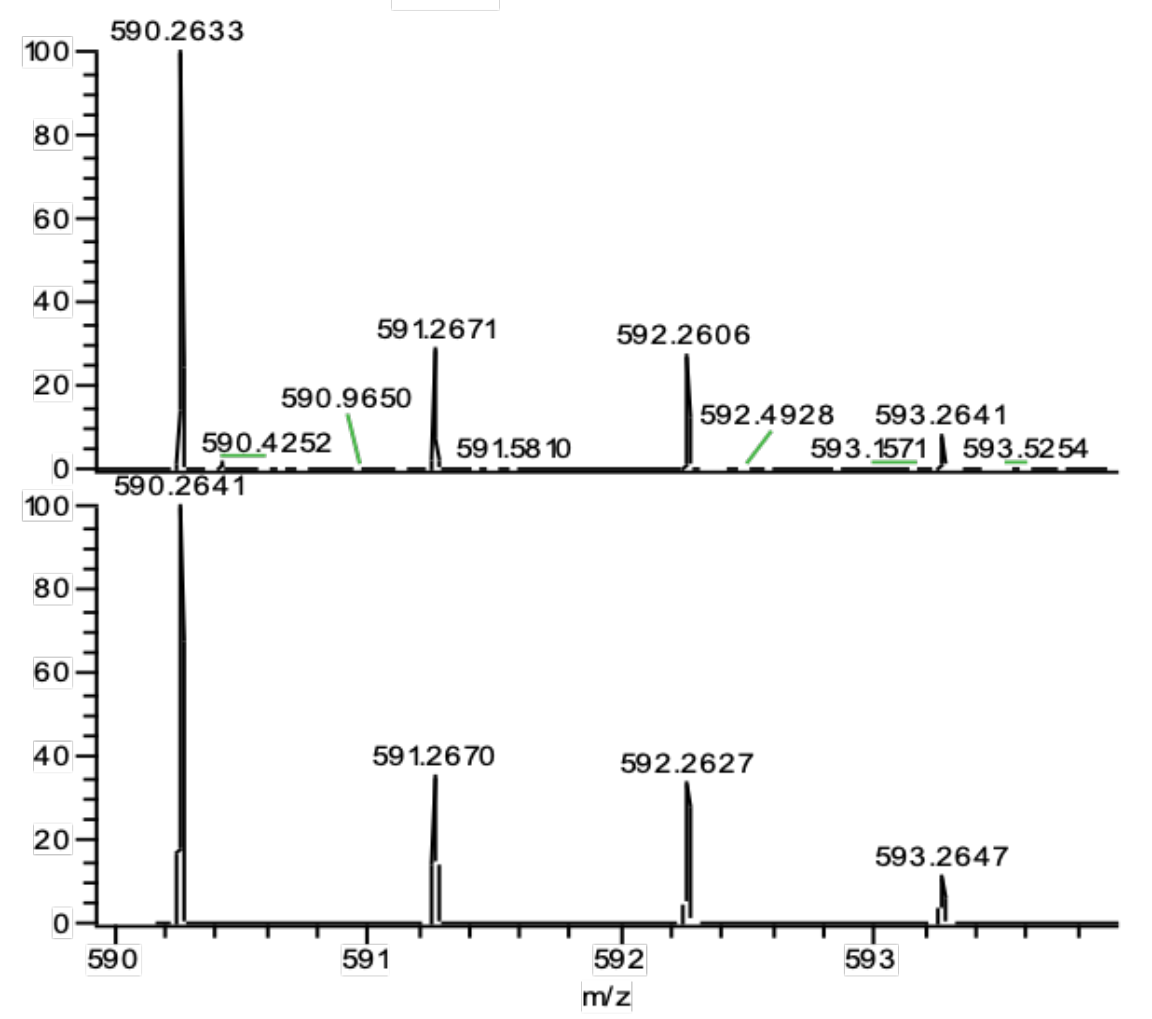

NL:

2.86E5

Linglan_20190215_5-69_2\#3-

$11 \mathrm{RT}: \overline{0} .11-0.49 \overline{\mathrm{AV}}: 9 \overline{\mathrm{T}}$ :

FTMS + p ESI Full ms

[100.00-1500.00]

NL:

1.23E4

$\mathrm{C}_{31} \mathrm{H}_{36} \mathrm{CIN}_{7} \mathrm{O}_{3} \mathrm{H}$ :

$\mathrm{C}_{31} \mathrm{H}_{37} \mathrm{Cl}_{1} \mathrm{~N}_{7} \mathrm{O}_{3}$

p (gss, s /p:40) Chrg 1

R: 45000 Res .Pwr . @FWHM 
(E)-cyclooct-4-en-1-yl (3-(4-amino-6-((E)-2-cyano-3-(isopropylamino)-3-oxoprop-1-en-1-yl)5-(4-phenoxyphenyl)-7H-pyrrolo[2,3-d]pyrimidin-7-yl)propyl)carbamate or (E)-cyclooct-4en-1-yl (3-(4-amino-6-((Z)-2-cyano-3-(isopropylamino)-3-oxoprop-1-en-1-yl)-5-(4phenoxyphenyl)-7H-pyrrolo[2,3- $d]$ pyrimidin-7-yl)propyl)carbamate (2-TCO)

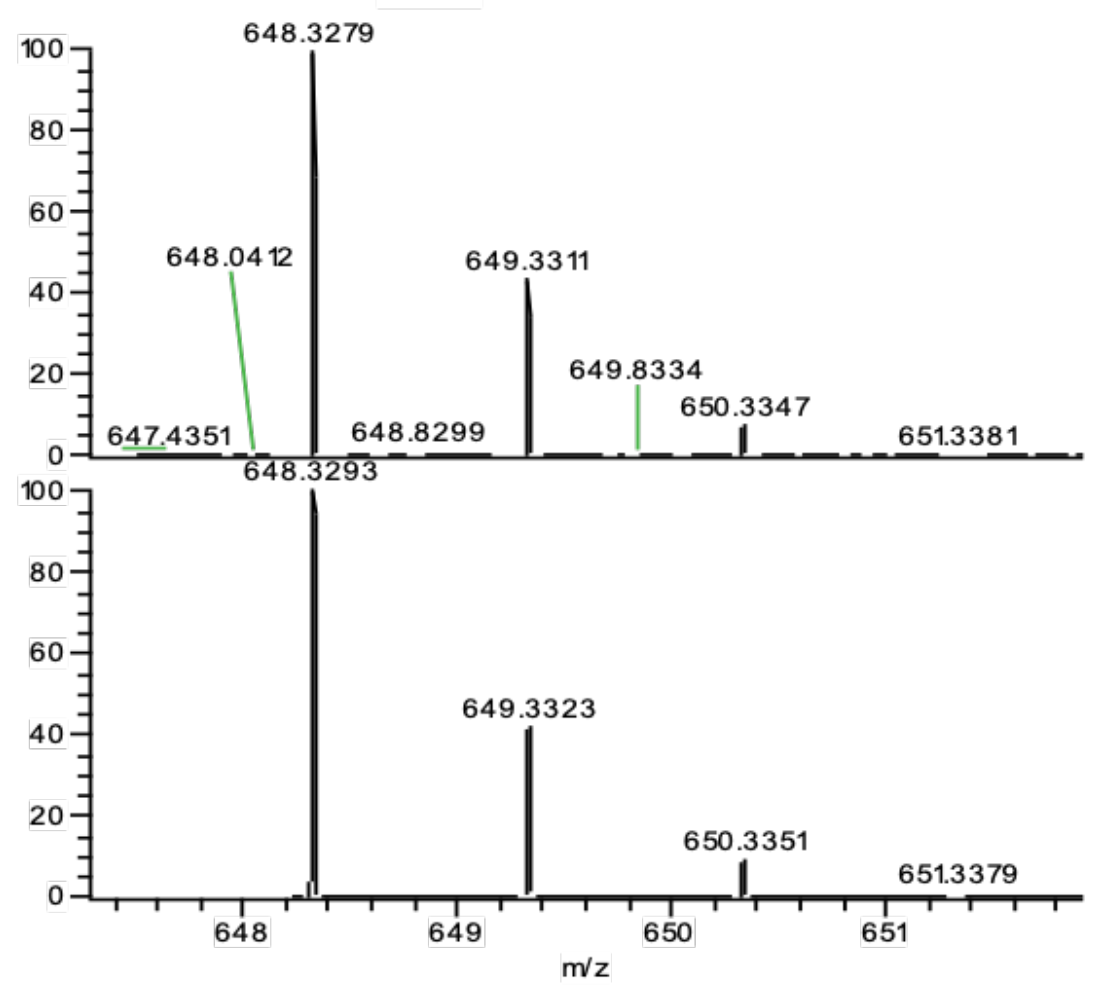

NL:

2.48E6

Linglan_20190215_594 \#35-357 RT: $3.32-4.37$ AV: 23 T: FTMS + p ESI Full $\mathrm{ms}$ [100.00-1500.00]

NL:

1.51E4

$\mathrm{C}_{37} \mathrm{H}_{41} \mathrm{~N}_{7} \mathrm{O}_{4} \mathrm{H}$ :

$\mathrm{C}_{37} \mathrm{H}_{42} \mathrm{~N}_{7} \mathrm{O}_{4}$

p (gss, s /p:40) Chrg 1

R: 45000 Res .Pwr . @FWHM 


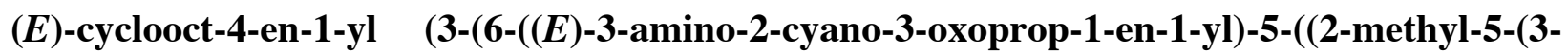
(trifluoromethyl)benzamido)phenyl)ethynyl)-7H-pyrrolo[2,3- $d]$ pyrimidin-7-

yl)propyl)carbamate or $(E)$-cyclooct-4-en-1-yl (3-(6-((Z)-3-amino-2-cyano-3-oxoprop-1-en1-yl)-5-((2-methyl-5-(3-(trifluoromethyl)benzamido)phenyl)ethynyl)-7H-pyrrolo[2,3-

d]pyrimidin-7-yl)propyl)carbamate (3-TCO)

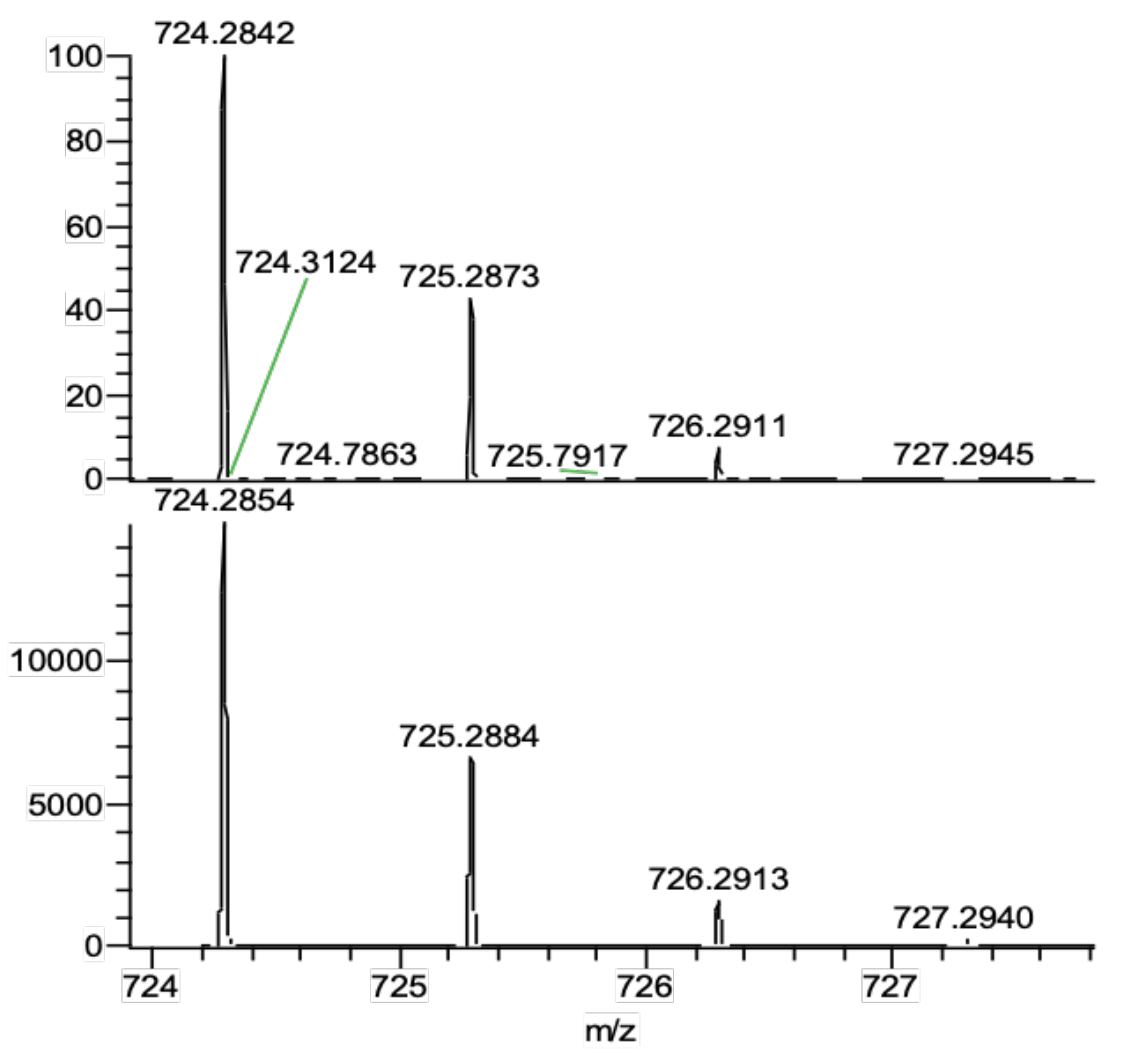

NL:

1.49E6

Linglan_20190215_5-

95 1\#411-422 RT: 3.49-4.02

AV: 12 T: FTMS + p ESI Full ms [100.00-1500.00]

NL:

1.48E4

$\mathrm{C}_{39} \mathrm{H}_{36} \mathrm{~F}_{3} \mathrm{~N}_{7} \mathrm{O}_{4} \mathrm{H}$ :

$\mathrm{C}_{39} \mathrm{H}_{37} \mathrm{~F}_{3} \mathrm{~N}_{7} \mathrm{O}_{4}$

p (gss, s /p:40) Chrg 1

R: 40000 Res .Pw r . @FWHM 


\section{NMR spectra:}

\section{Tert-butyl (3-bromopropyl)carbamate (4)}

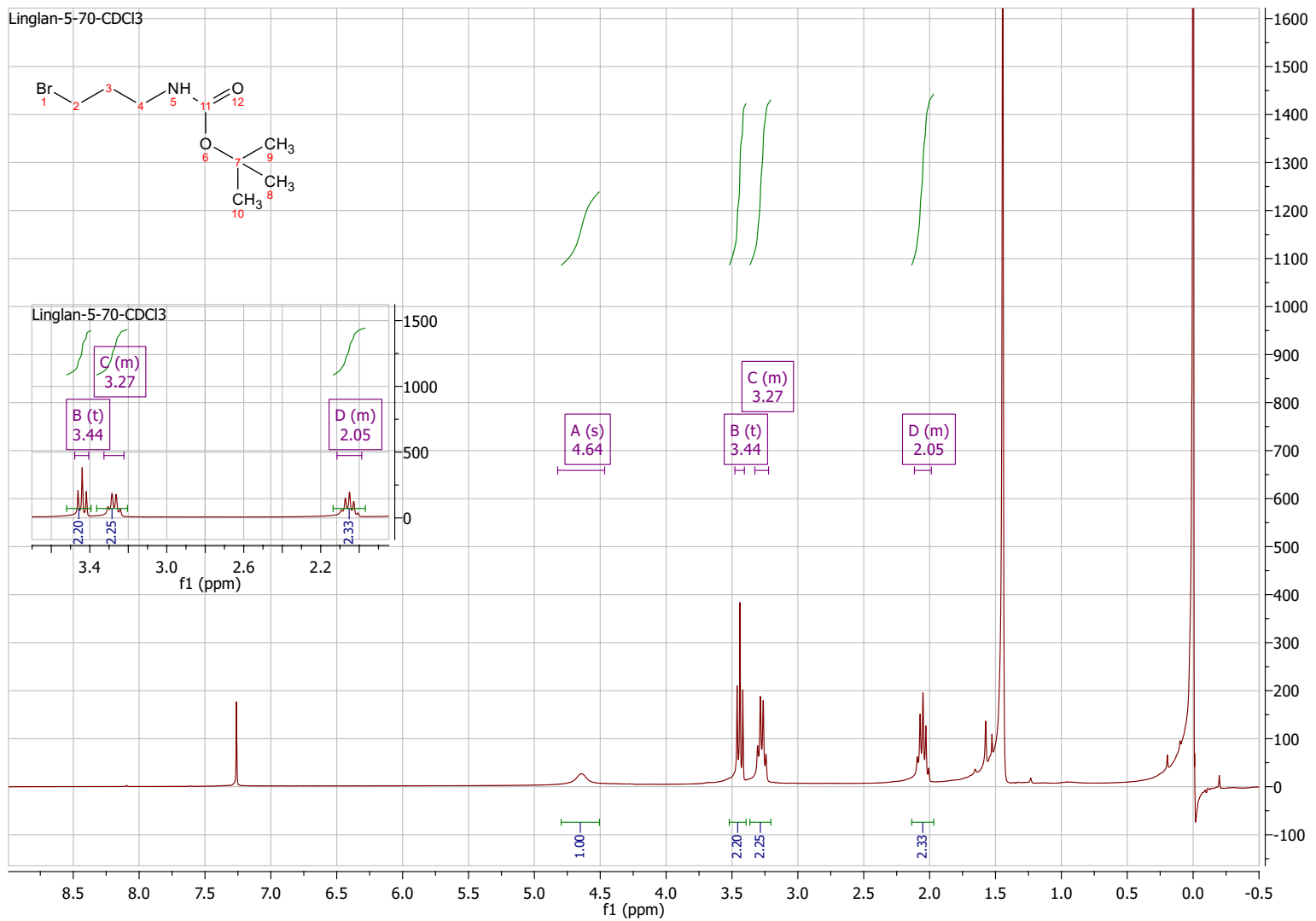


Tert-butyl (3-(4-chloro-5-iodo-7H-pyrrolo[2,3-d]pyrimidin-7-yl)propyl)carbamate (5)

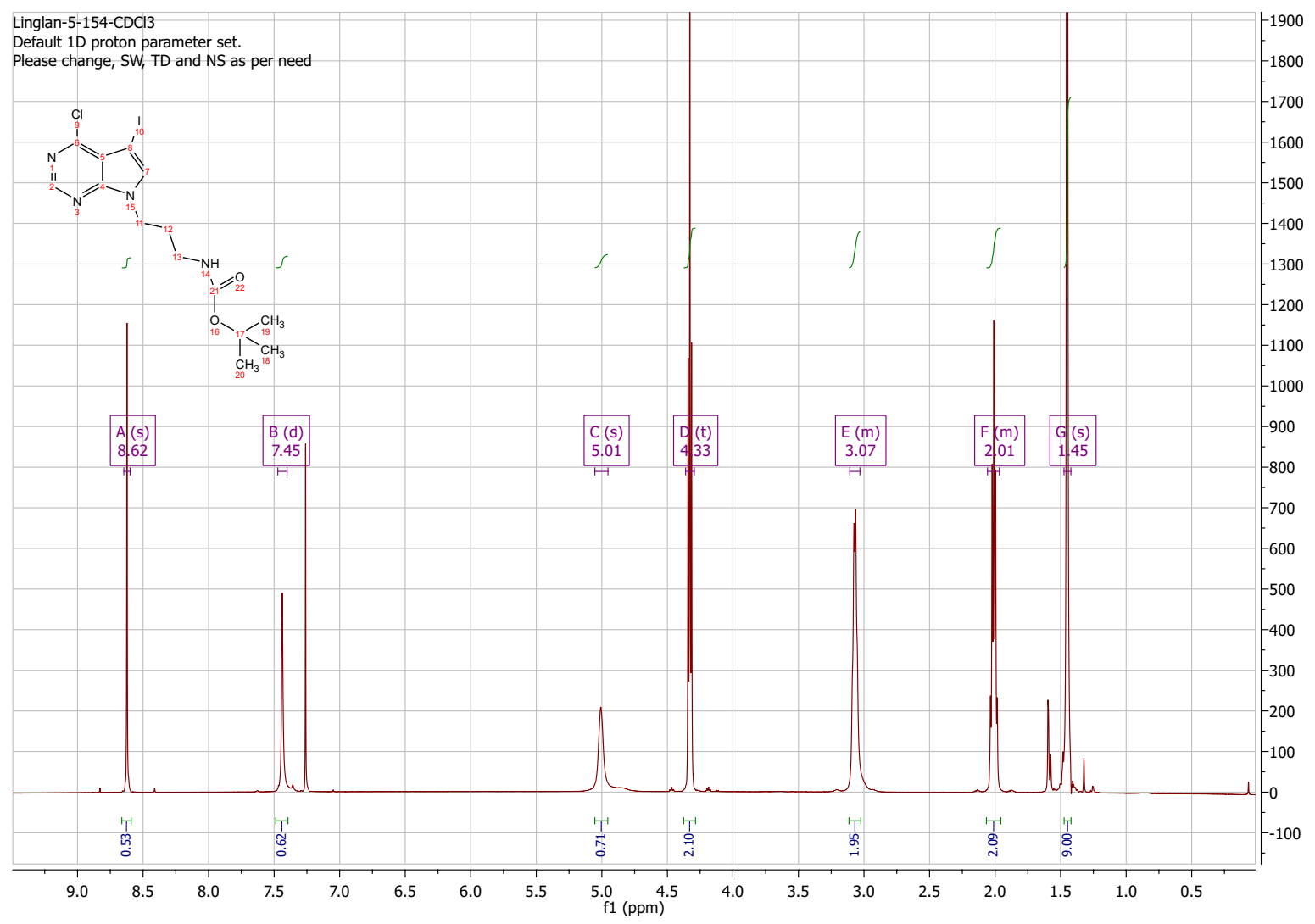


Tert-butyl (3-(4-chloro-6-formyl-5-iodo-7H-pyrrolo[2,3- $d]$ pyrimidin-7-yl)propyl)carbamate

(6)

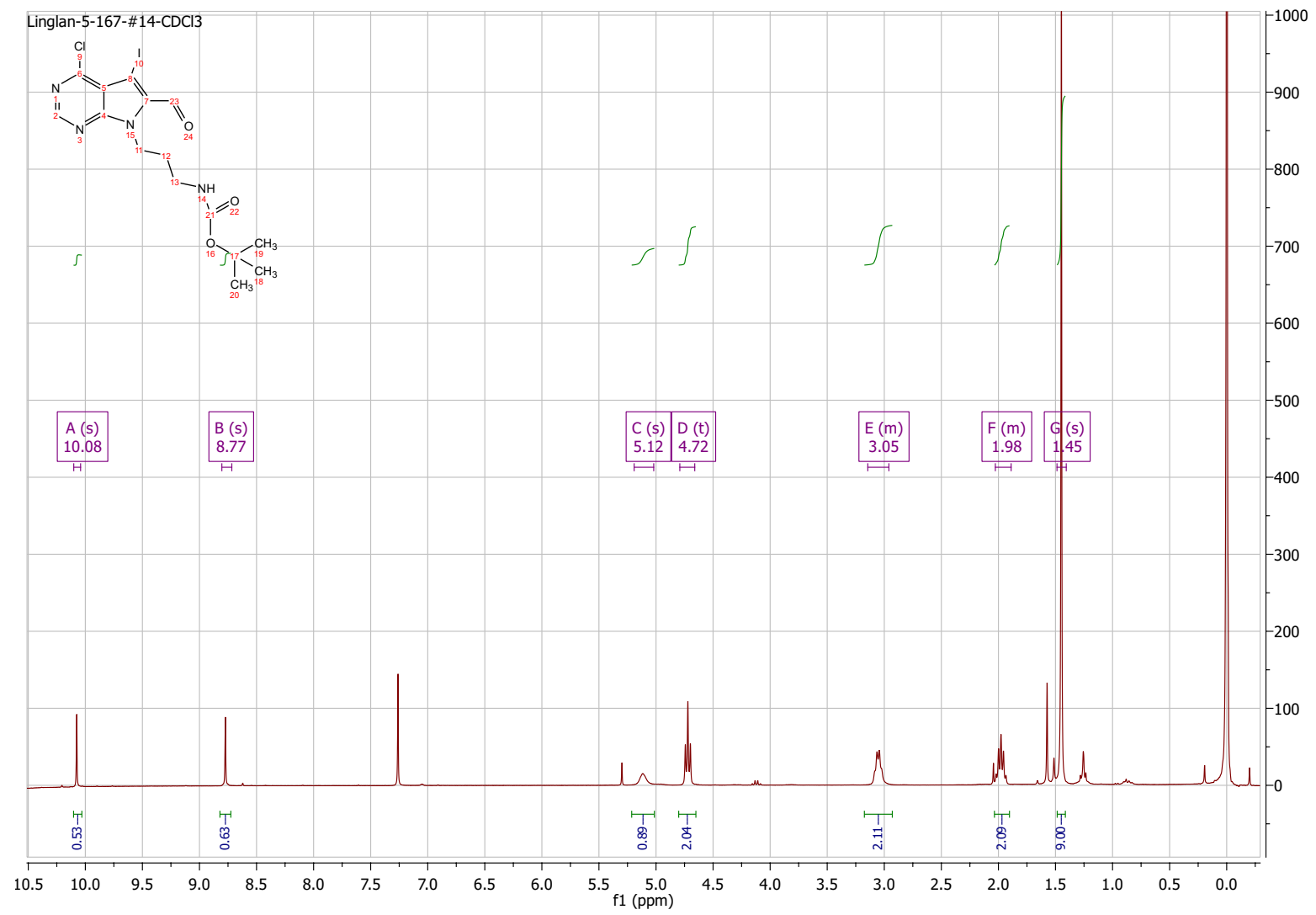


Tert-butyl (3-(4-amino-6-formyl-5-iodo-7H-pyrrolo[2,3- $d]$ pyrimidin-7-yl)propyl)carbamate

(7)

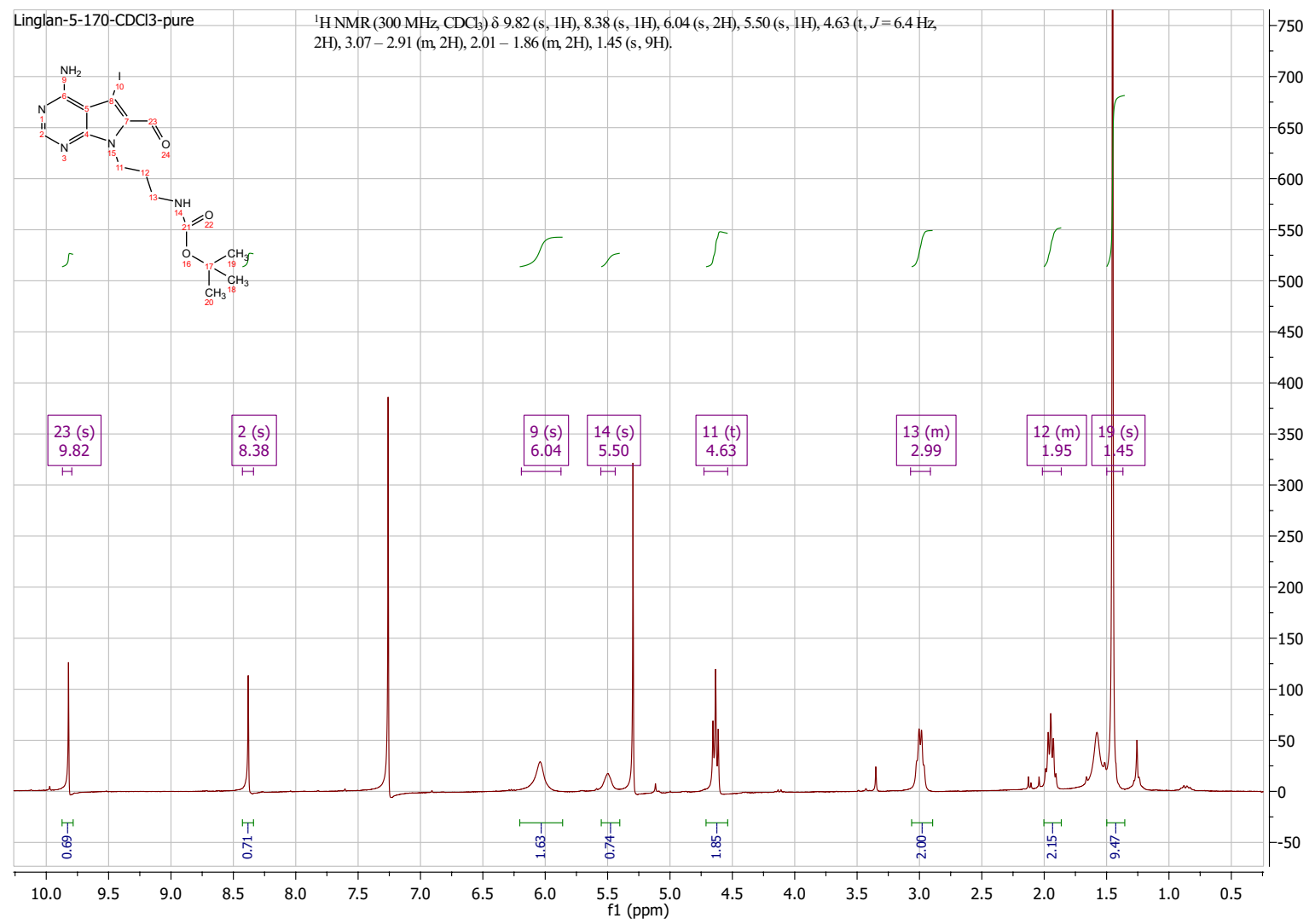


yl)propyl)carbamate (8)

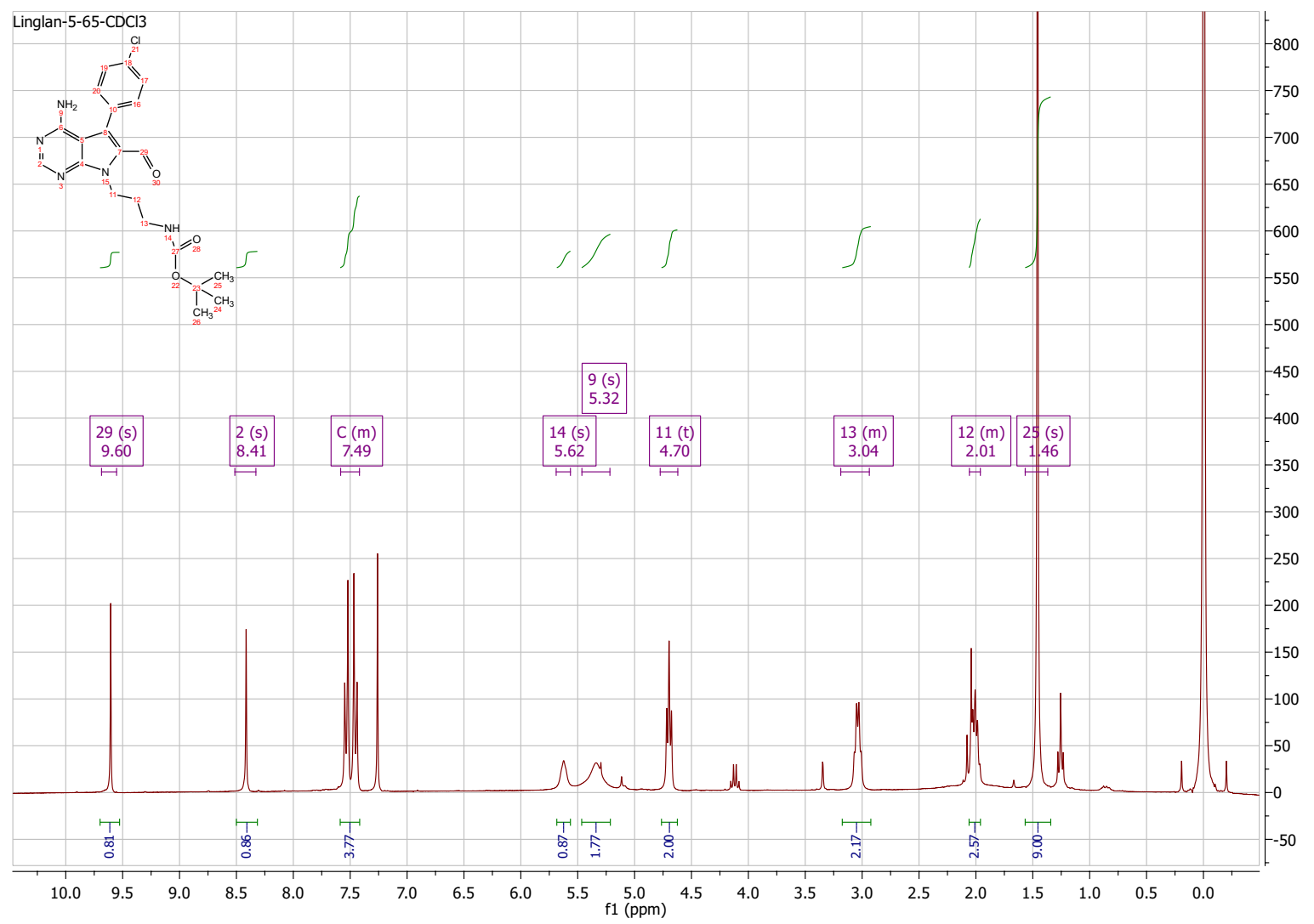


(Z)- or (E)-tert-butyl (3-(4-amino-5-(4-chlorophenyl)-6-(2-cyano-3-(isopropylamino)-3oxoprop-1-en-1-yl)-7H-pyrrolo[2,3- $d]$ pyrimidin-7-yl)propyl)carbamate (9)

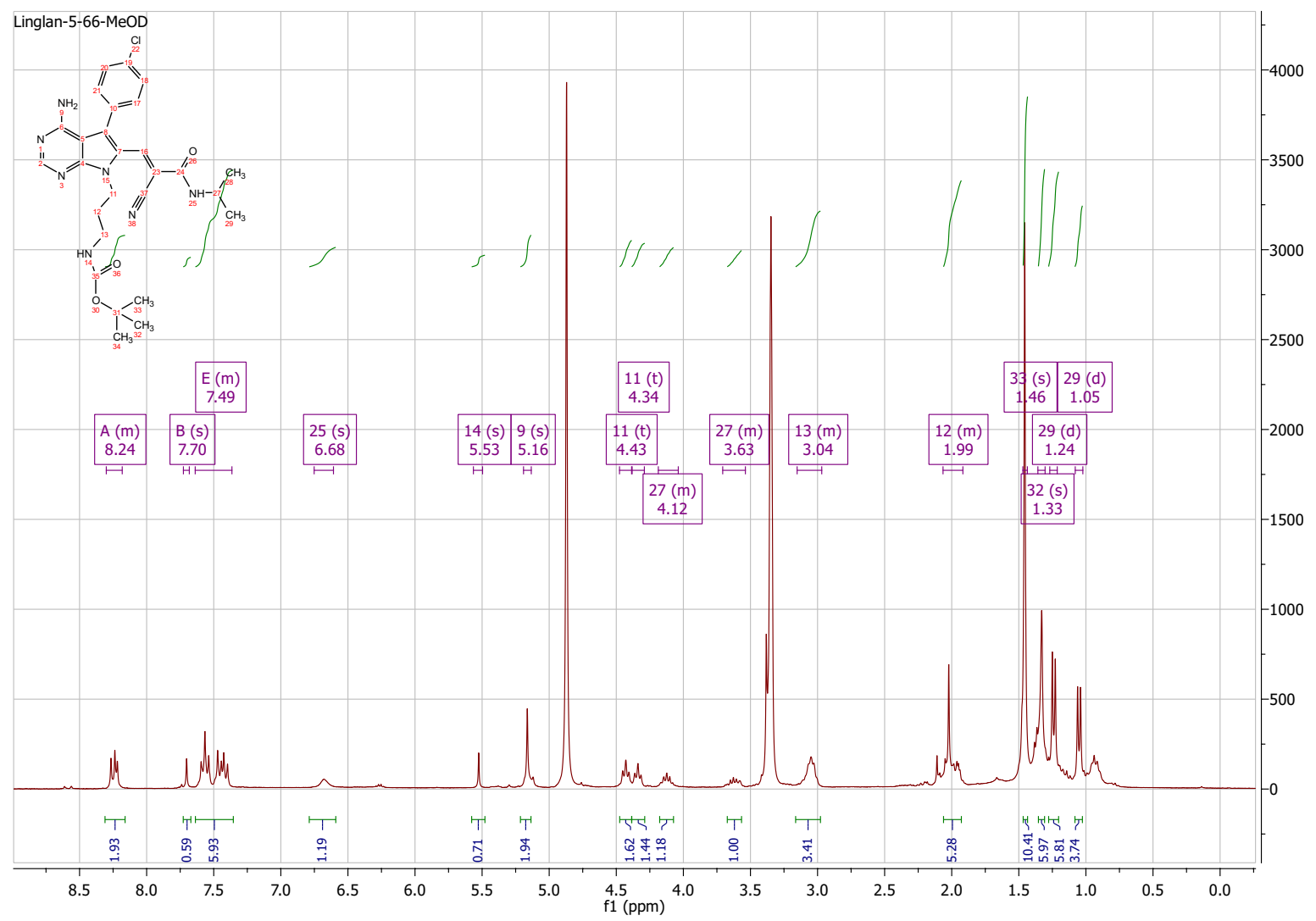


(E)-cyclooct-4-en-1-yl (3-(4-amino-5-(4-chlorophenyl)-6-((E)-2-cyano-3-(isopropylamino)-3oxoprop-1-en-1-yl)-7H-pyrrolo[2,3-d]pyrimidin-7-yl)propyl)carbamate or (E)-cyclooct-4en-1-yl (3-(4-amino-5-(4-chlorophenyl)-6-((Z)-2-cyano-3-(isopropylamino)-3-oxoprop-1-en1-yl)-7H-pyrrolo[2,3-d]pyrimidin-7-yl)propyl)carbamate (1-TCO)

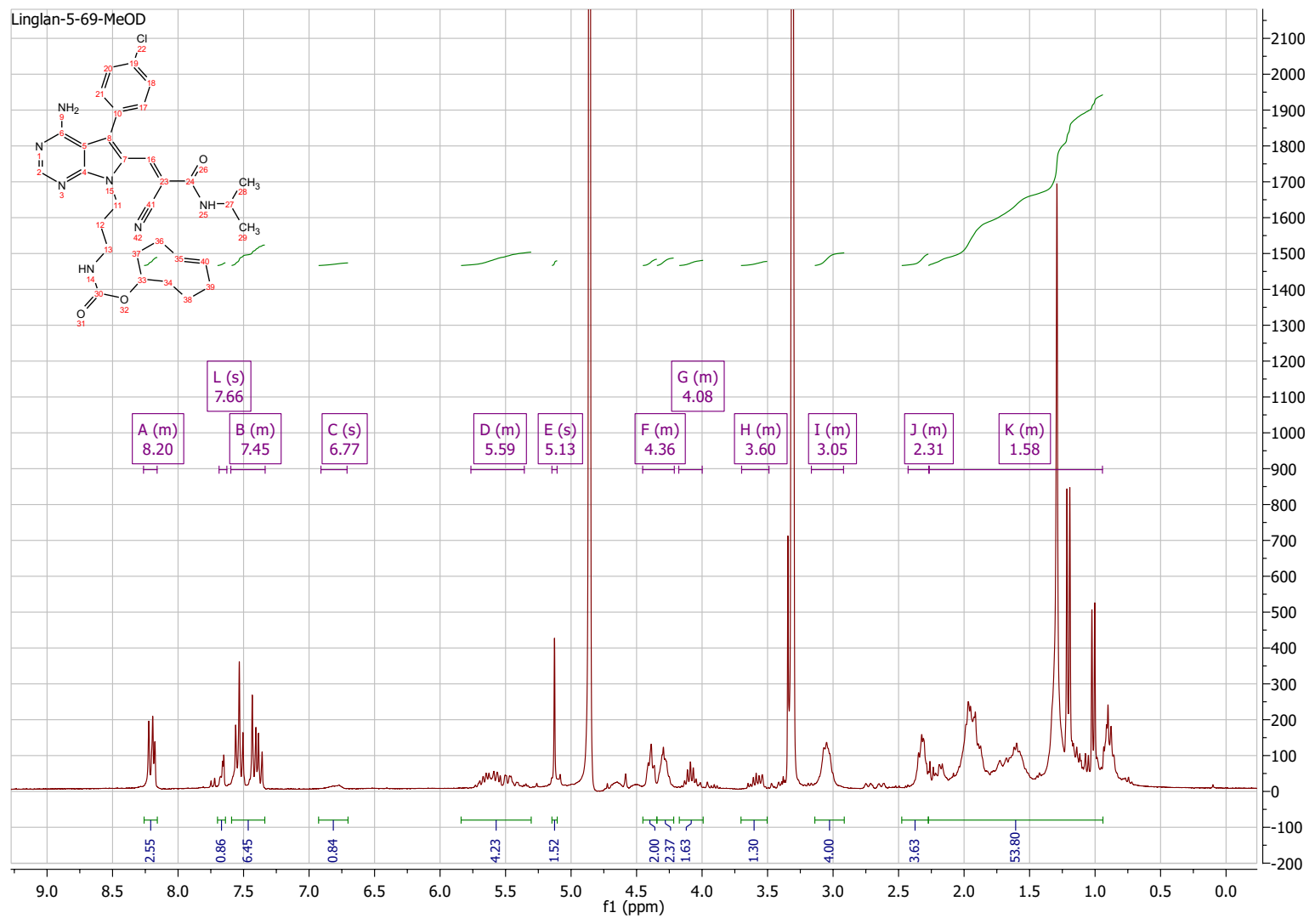


Tert-butyl (3-(4-amino-6-formyl-5-(4-phenoxyphenyl)-7H-pyrrolo[2,3- $d]$ pyrimidin-7yl)propyl)carbamate

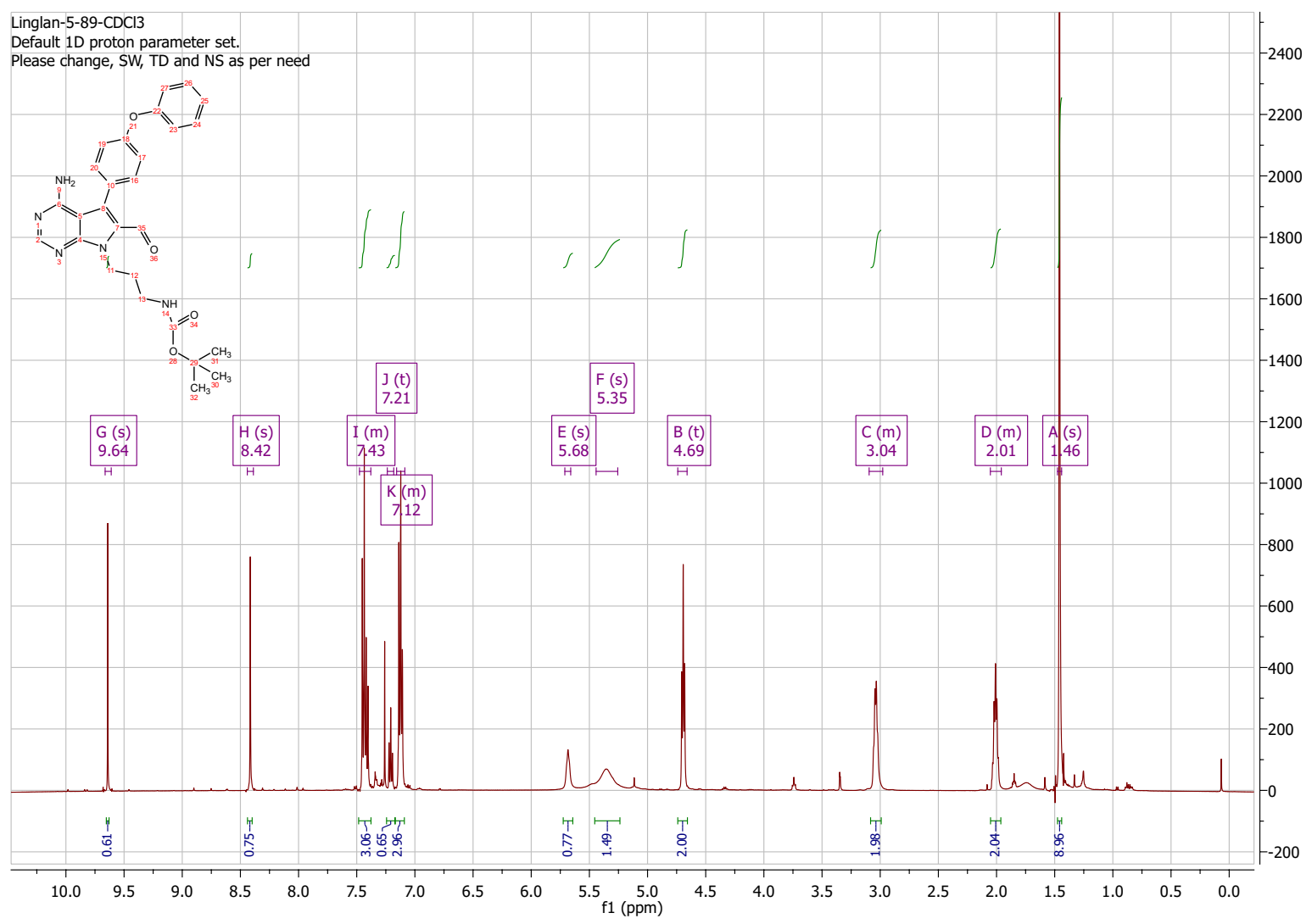


(Z)- or $(E)$-tert-butyl (3-(4-amino-6-(2-cyano-3-(isopropylamino)-3-oxoprop-1-en-1-yl)-5-(4phenoxyphenyl)-7H-pyrrolo[2,3- $d]$ pyrimidin-7-yl)propyl)carbamate (11)

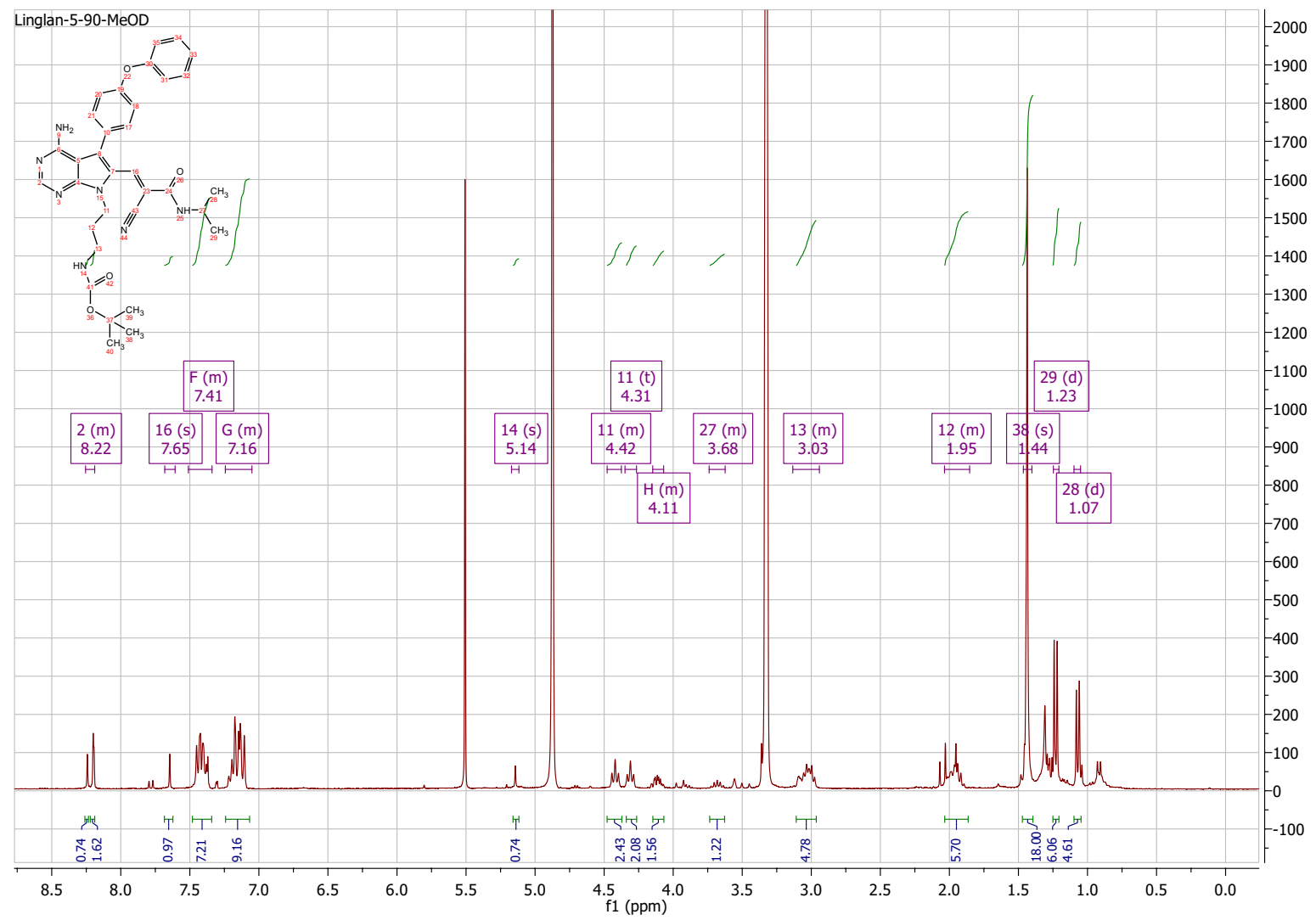


(E)-cyclooct-4-en-1-yl (3-(4-amino-6-((E)-2-cyano-3-(isopropylamino)-3-oxoprop-1-en-1-yl)5-(4-phenoxyphenyl)-7H-pyrrolo[2,3-d]pyrimidin-7-yl)propyl)carbamate or (E)-cyclooct-4en-1-yl (3-(4-amino-6-((Z)-2-cyano-3-(isopropylamino)-3-oxoprop-1-en-1-yl)-5-(4phenoxyphenyl)-7H-pyrrolo[2,3- $d]$ pyrimidin-7-yl)propyl)carbamate (2-TCO)

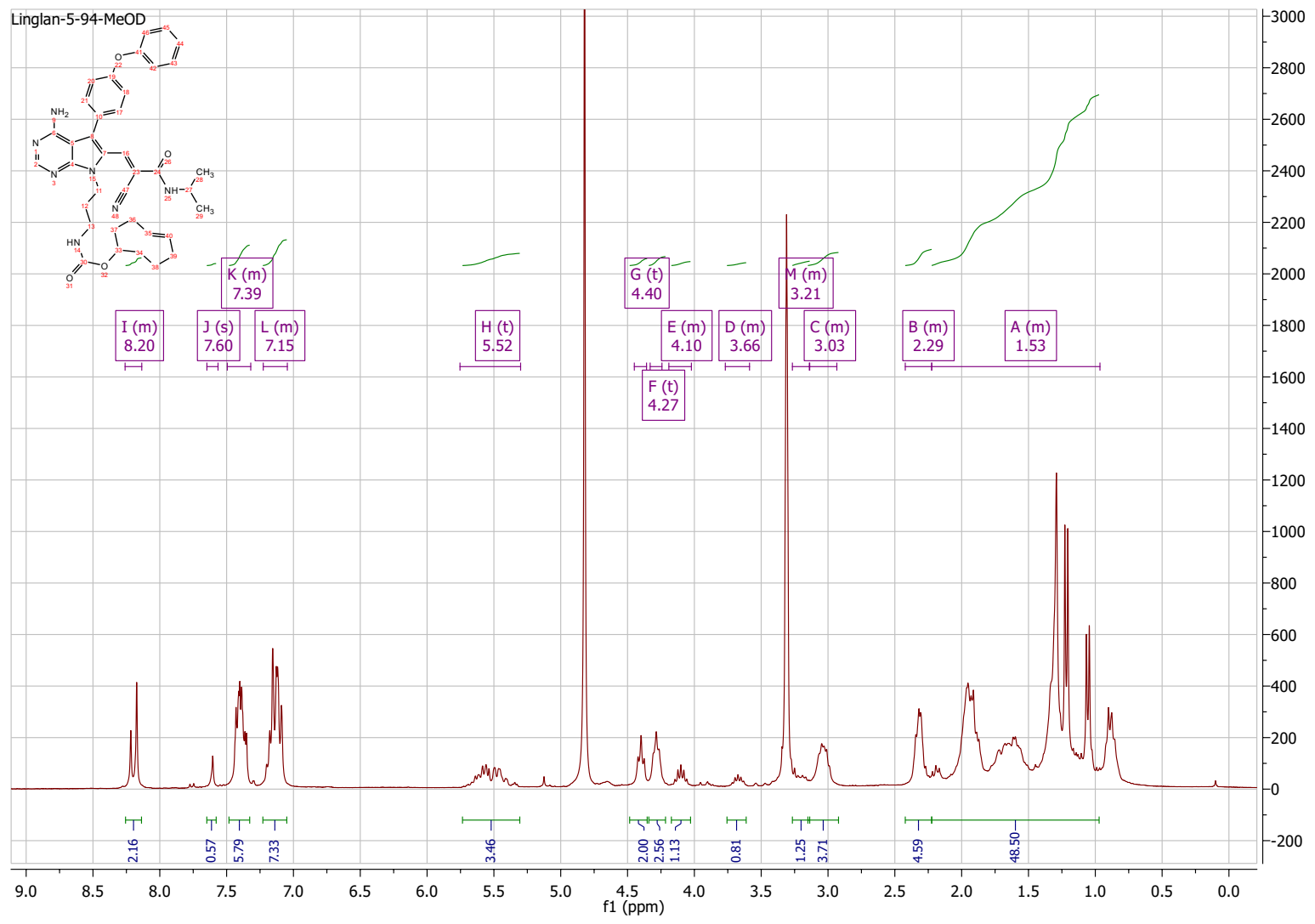


Tert-butyl (3-(5-iodo-7H-pyrrolo[2,3-d]pyrimidin-7-yl)propyl)carbamate (12)

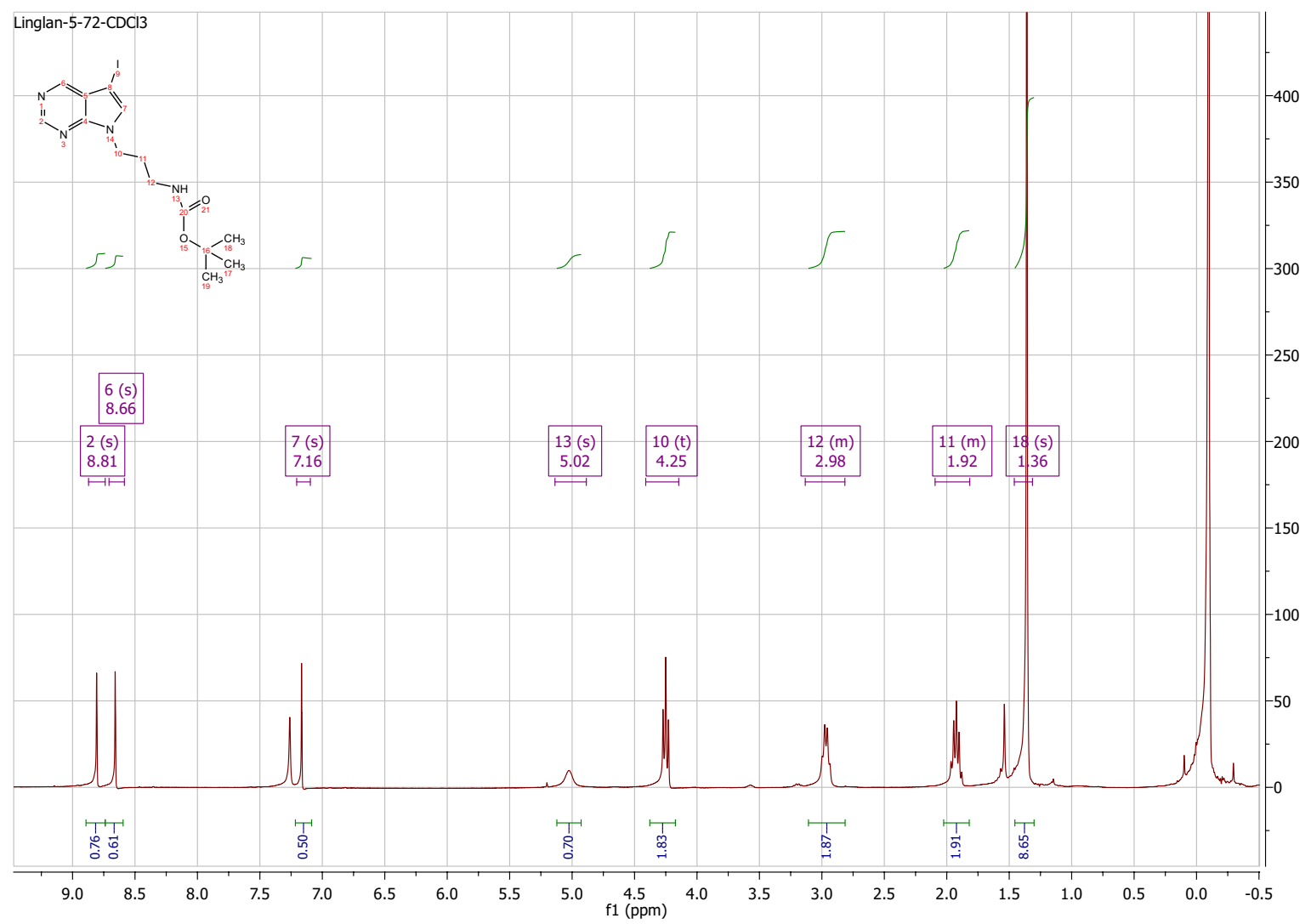


Tert-butyl (3-(6-formyl-5-iodo-7H-pyrrolo[2,3-d]pyrimidin-7-yl)propyl)carbamate (13)

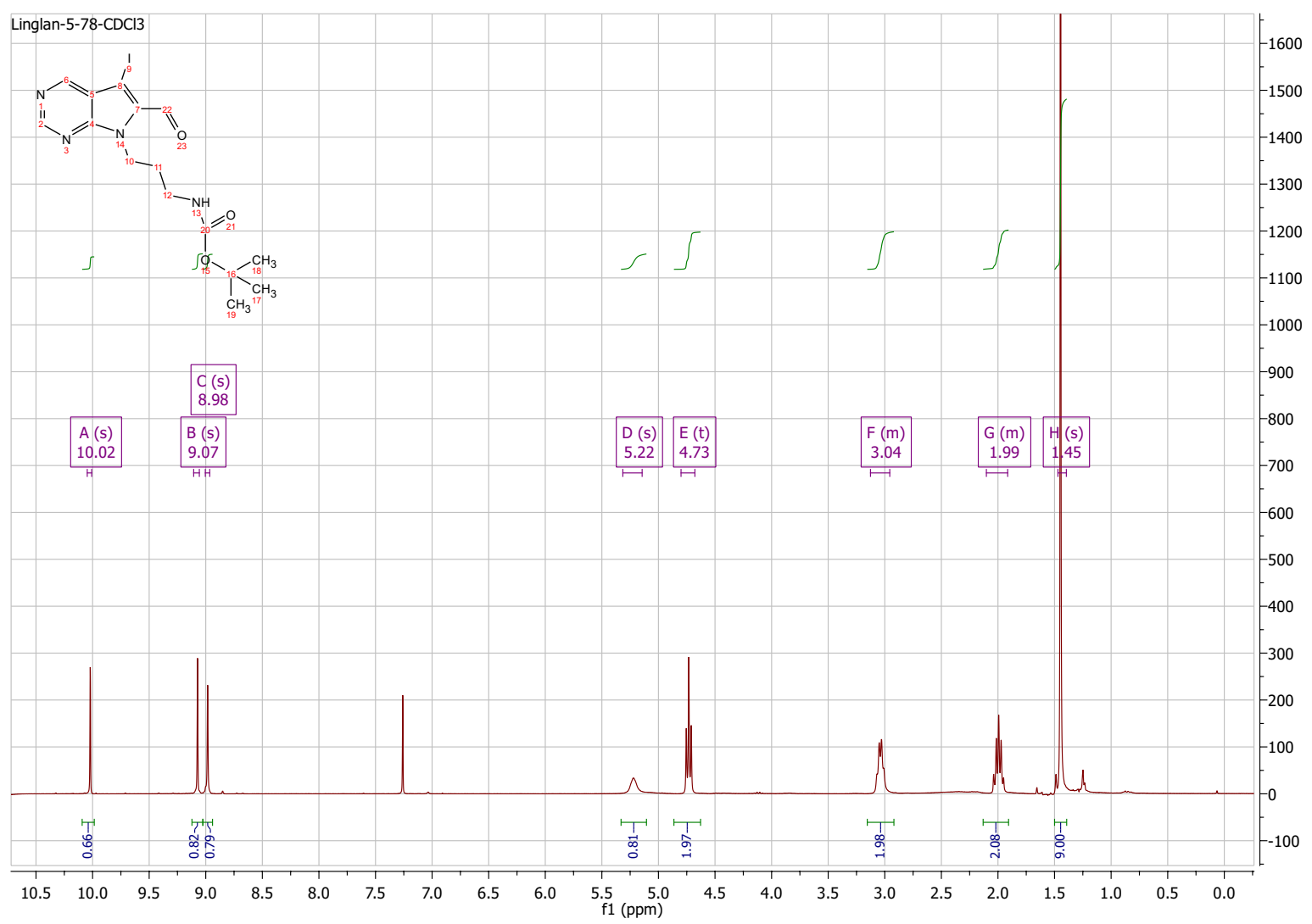


Tert-butyl (3-(6-formyl-5-((2-methyl-5-(3-(trifluoromethyl)benzamido)phenyl)ethynyl)-7Hpyrrolo[2,3-d]pyrimidin-7-yl)propyl)carbamate (14)

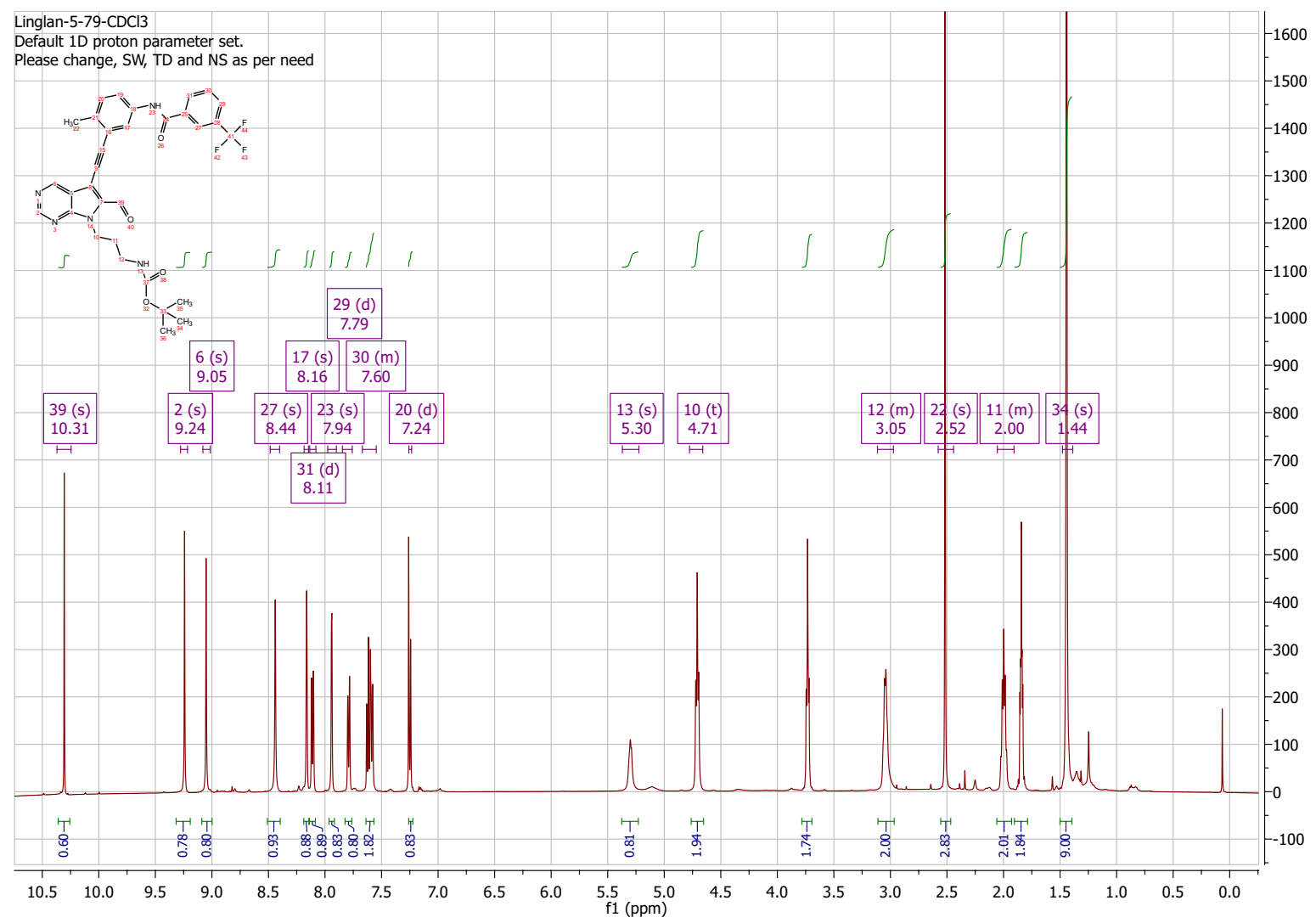


(Z)- or (E)-tert-butyl (3-(6-(3-amino-2-cyano-3-oxoprop-1-en-1-yl)-5-((2-methyl-5-(3(trifluoromethyl)benzamido)phenyl)ethynyl)-7H-pyrrolo[2,3- $d]$ pyrimidin-7-

\section{yl)propyl)carbamate (15)}

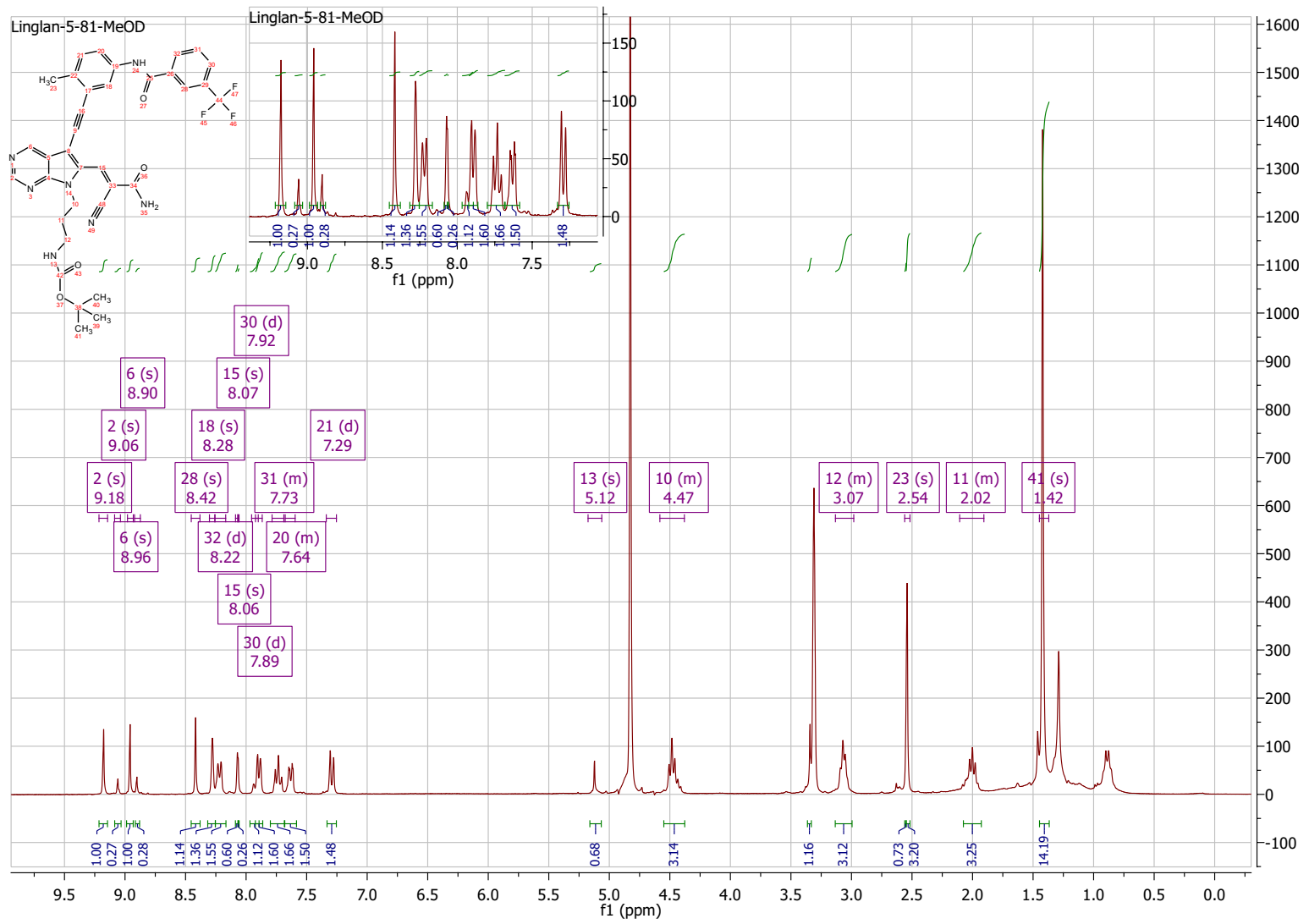


(E)-cyclooct-4-en-1-yl (3-(6-((E)-3-amino-2-cyano-3-oxoprop-1-en-1-yl)-5-((2-methyl-5-(3(trifluoromethyl)benzamido)phenyl)ethynyl)-7H-pyrrolo[2,3- $d]$ pyrimidin-7-

yl)propyl)carbamate or (E)-cyclooct-4-en-1-yl (3-(6-((Z)-3-amino-2-cyano-3-oxoprop-1-en-

1-yl)-5-((2-methyl-5-(3-(trifluoromethyl)benzamido)phenyl)ethynyl)-7H-pyrrolo[2,3-

d]pyrimidin-7-yl)propyl)carbamate (3-TCO)

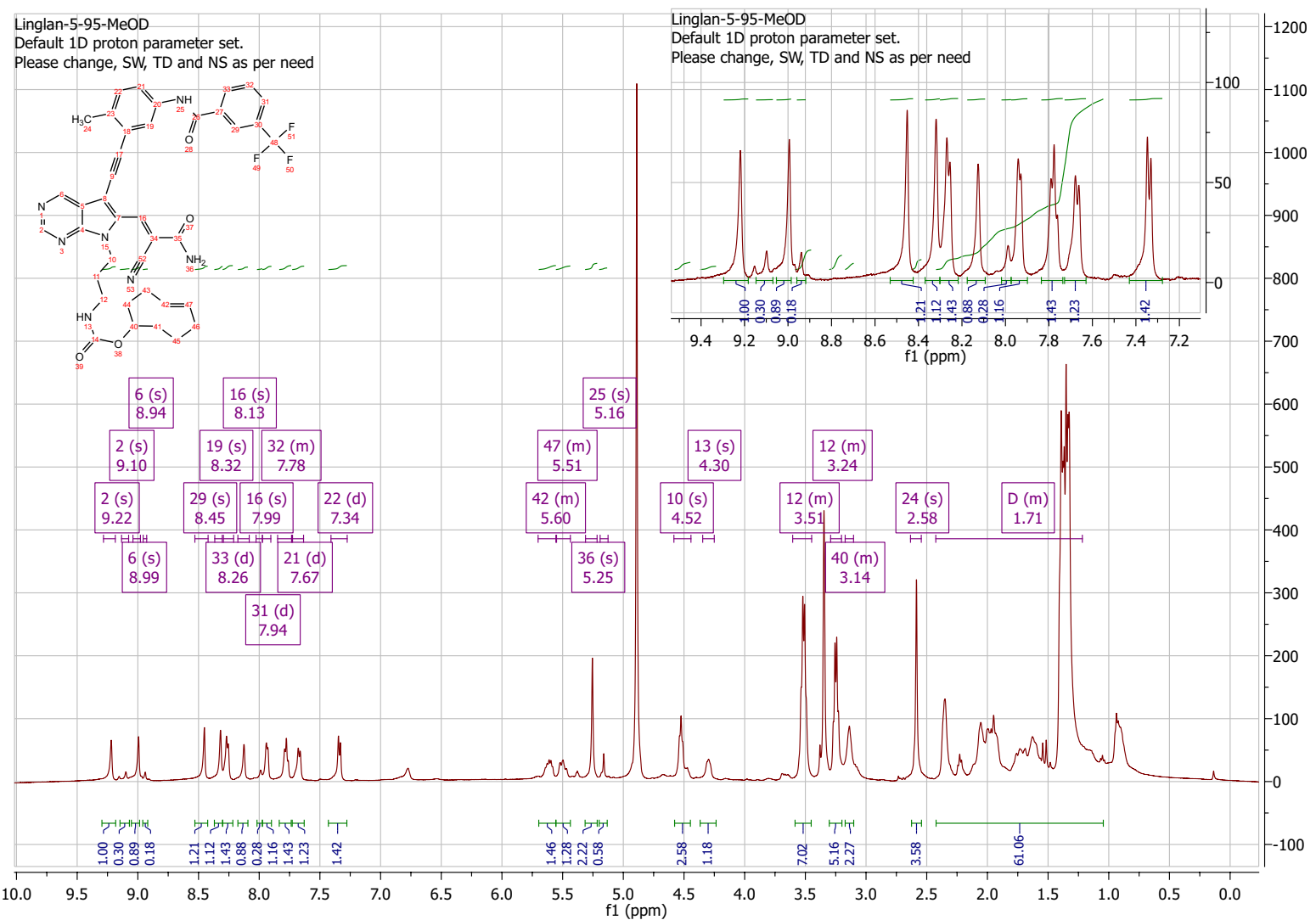


Tert-butyl (1-(4-(1,2,4,5-tetrazin-3-yl)phenyl)-2-oxo-6,9,12,15,18-pentaoxa-3-azaicosan-20yl)carbamate

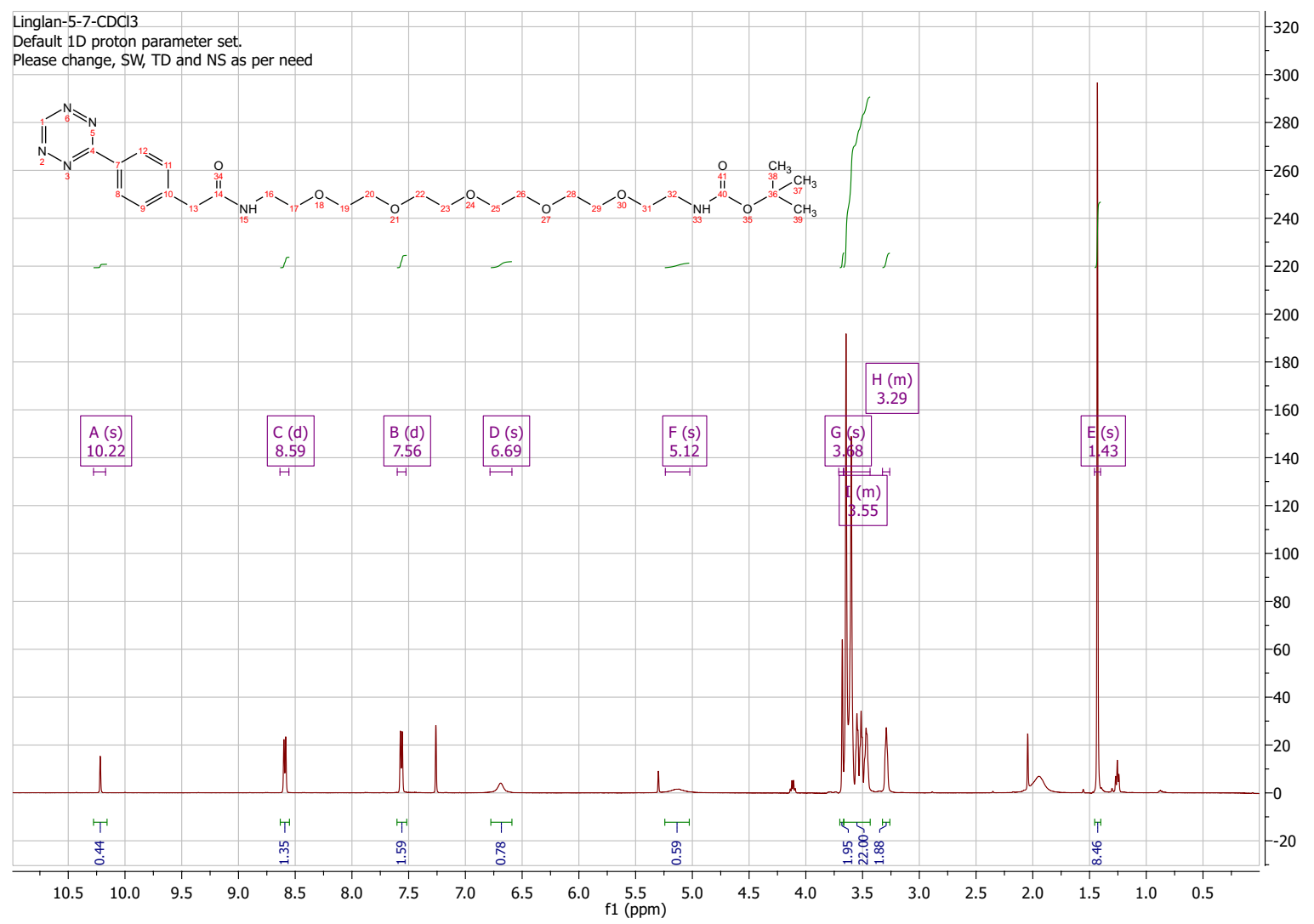


Immunoblots for Figure 2E (contain protein ladders)

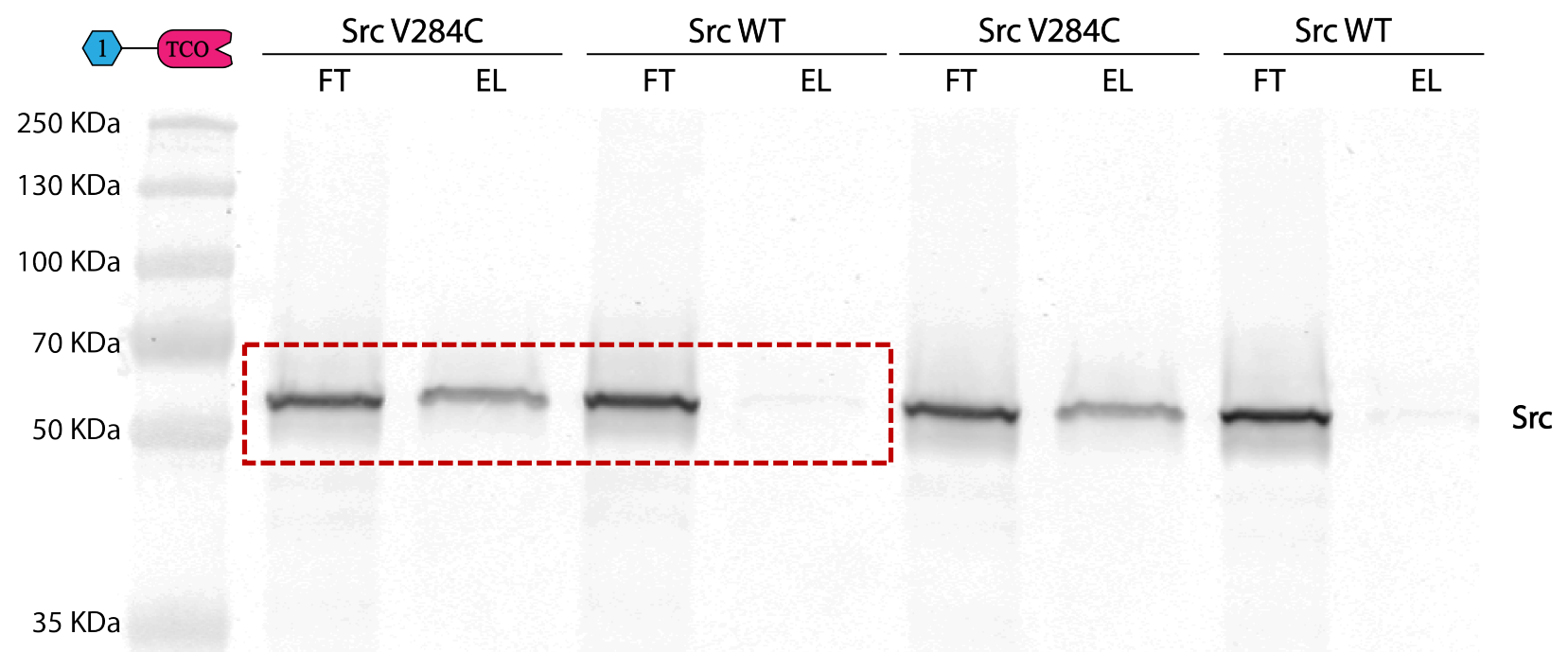

Immunoblots for Figure 3C (contain protein ladders)

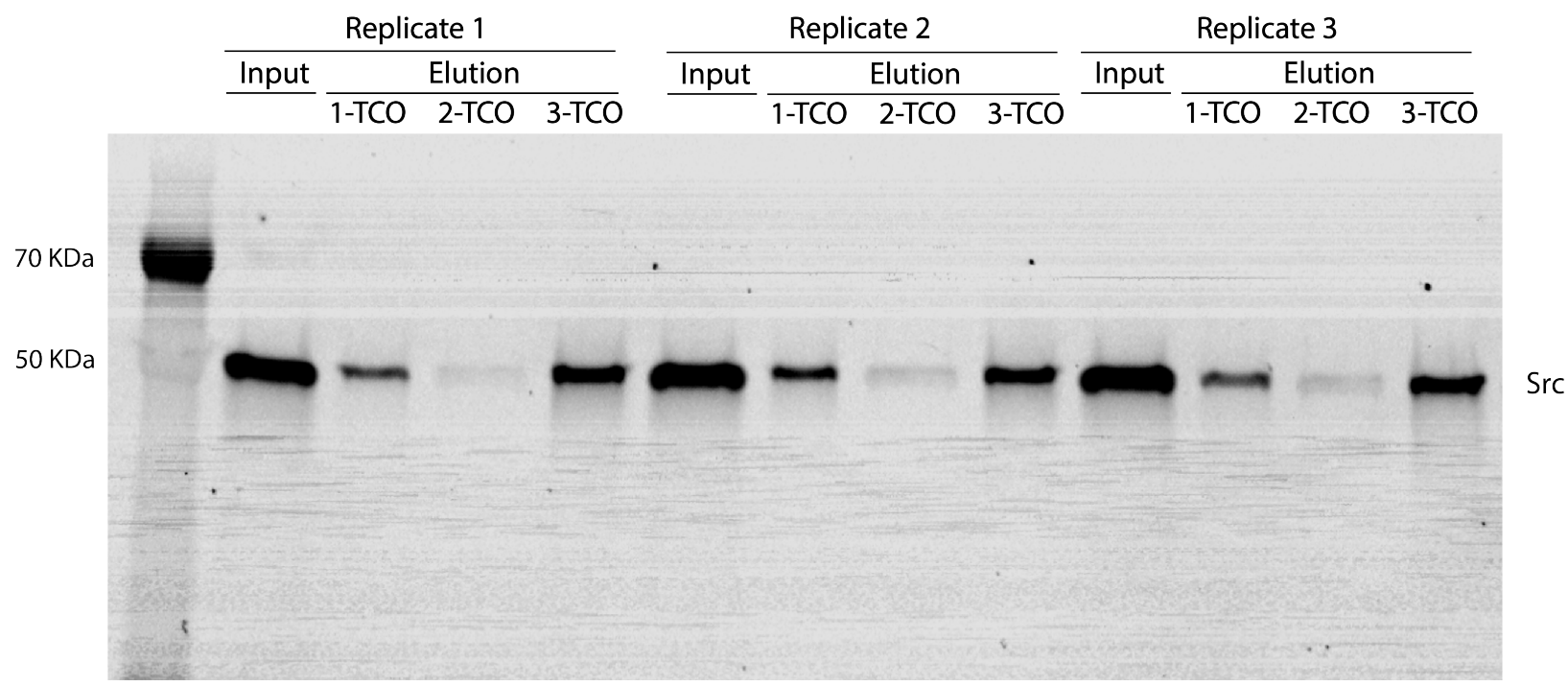


Immunoblots for Figure 3D (top) (contain protein ladders)

(2)-

$130 \mathrm{KDa}$

$100 \mathrm{KDa}$

$70 \mathrm{KDa}$

$50 \mathrm{KDa}$

$35 \mathrm{KDa}$

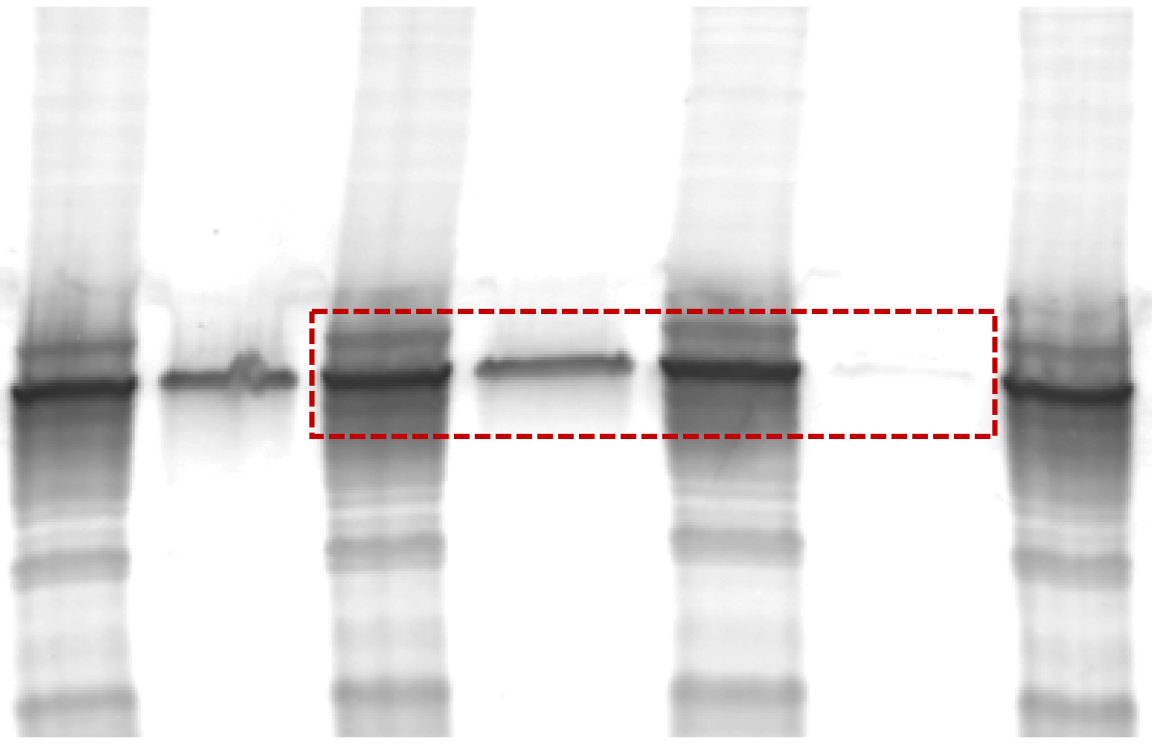

Src

Immunoblots for Figure 3D (bottom) (contain protein ladders)

(3)-

$130 \mathrm{KDa}$

$100 \mathrm{KDa}$

$70 \mathrm{KDa}$

$50 \mathrm{KDa}$

$35 \mathrm{KDa}$ 
Immunoblots for Figure 3F (contain protein ladders)

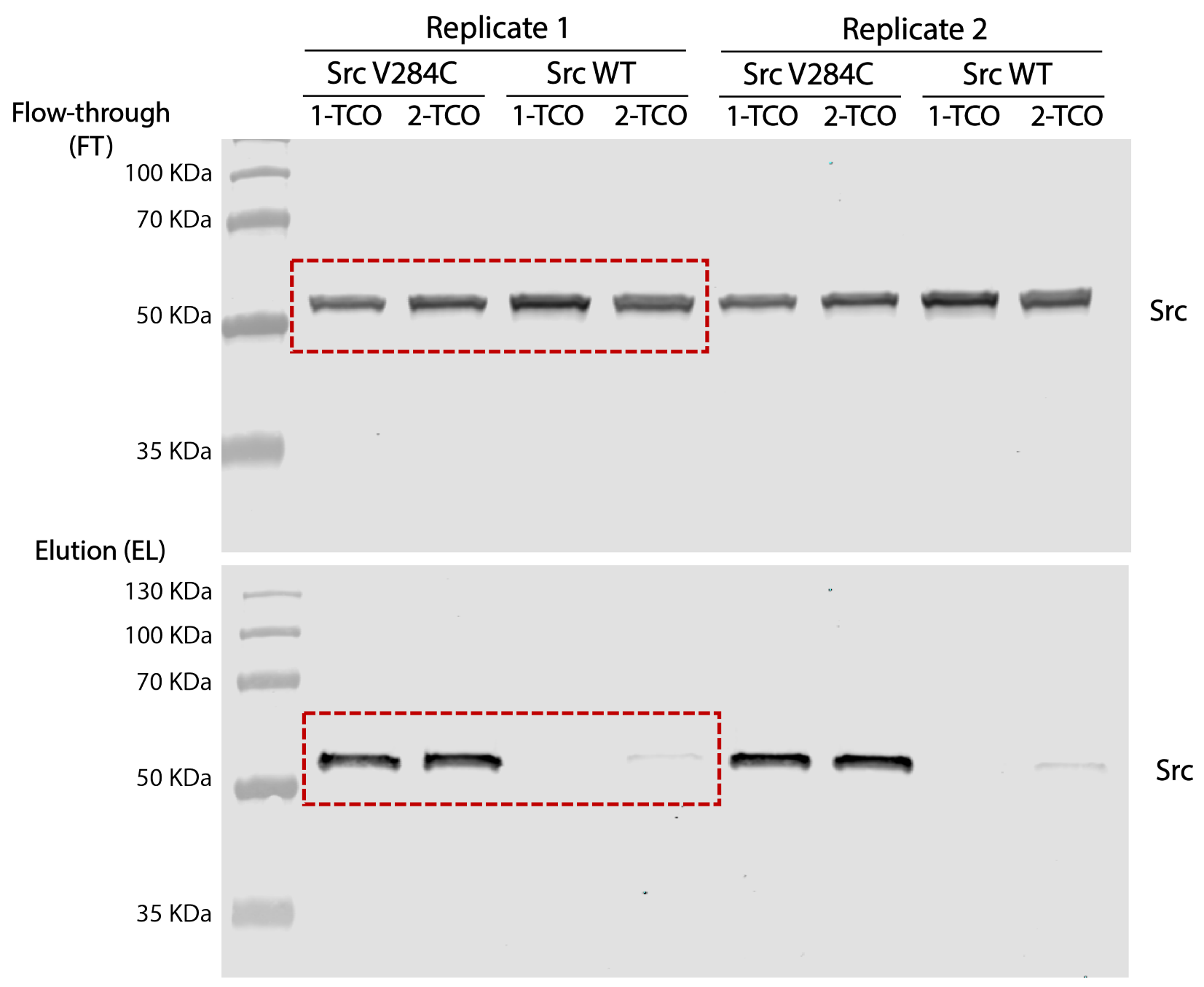


Immunoblots for Figure 3G (contain protein ladders)

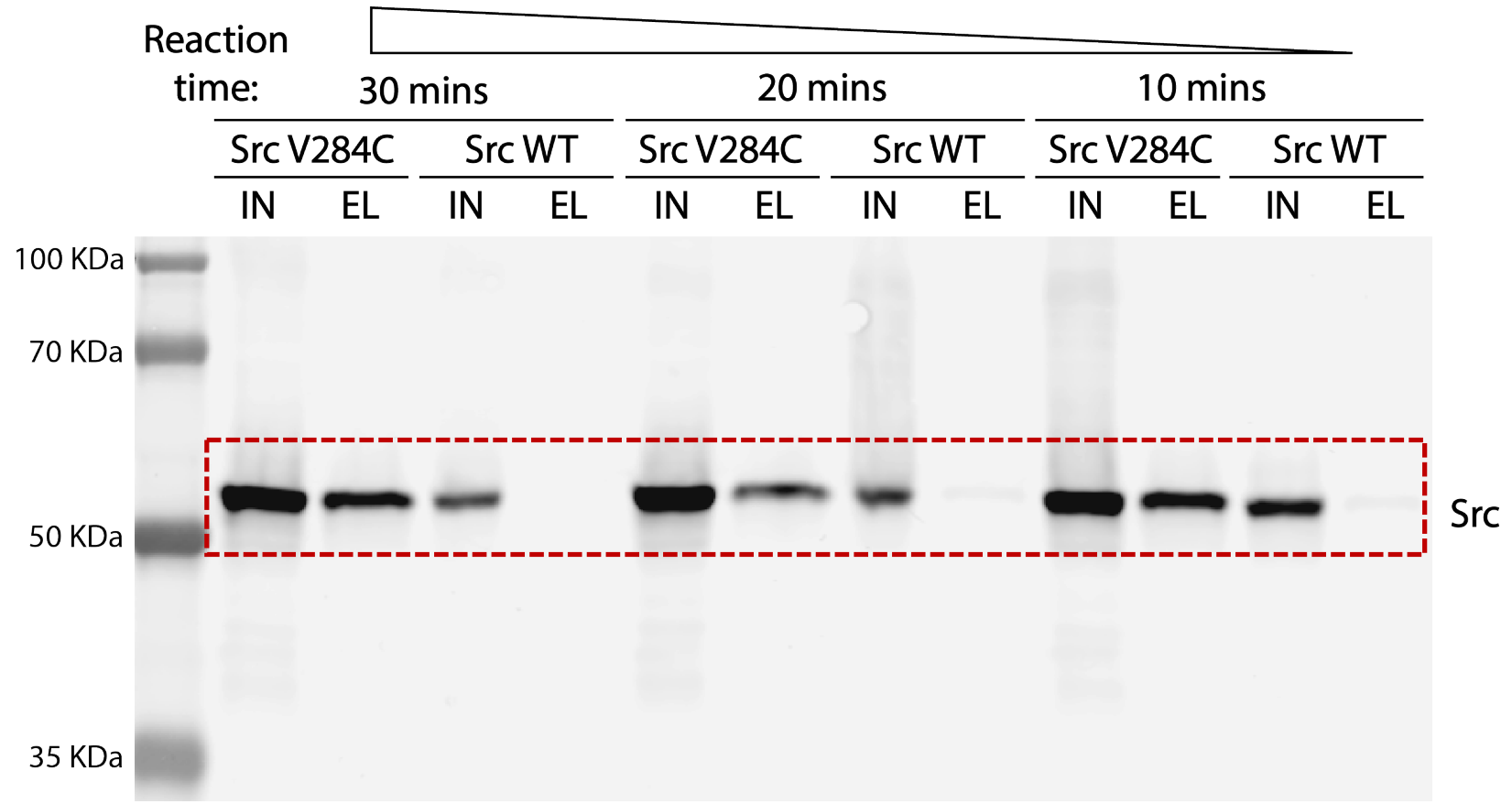

In vitro activity assay for determining IC50 values of 1, 2 and 3 against Src, EphA2 and JNK2. 
Immunoblots for Figure S6

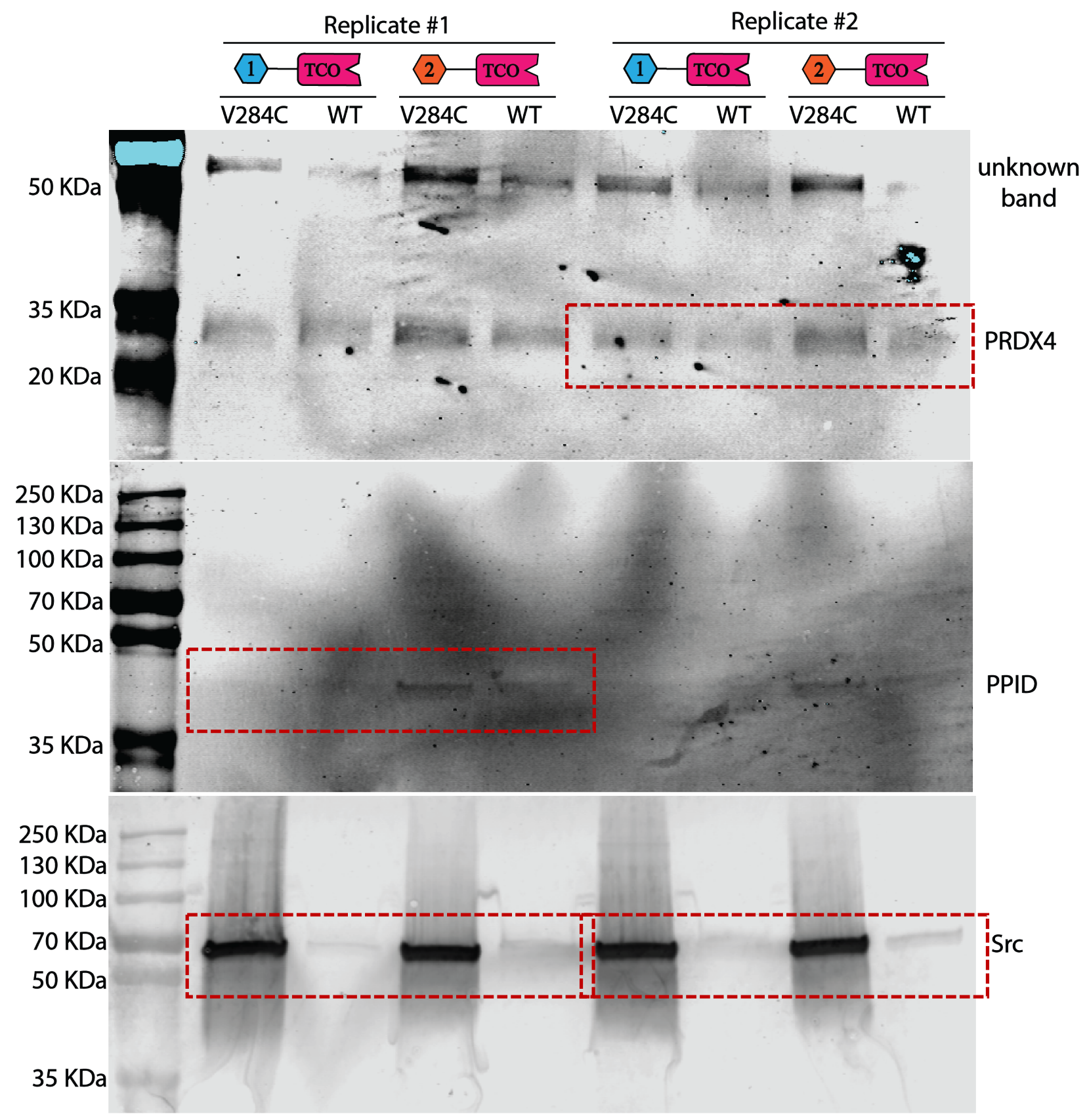


Immunoblots for Figure S3B

$\frac{\text { His-EphA2 V627C }}{\text { FT }} \frac{\text { His-EphA2 WT* }}{\text { EL }} \frac{\text { FT }}{E L}$

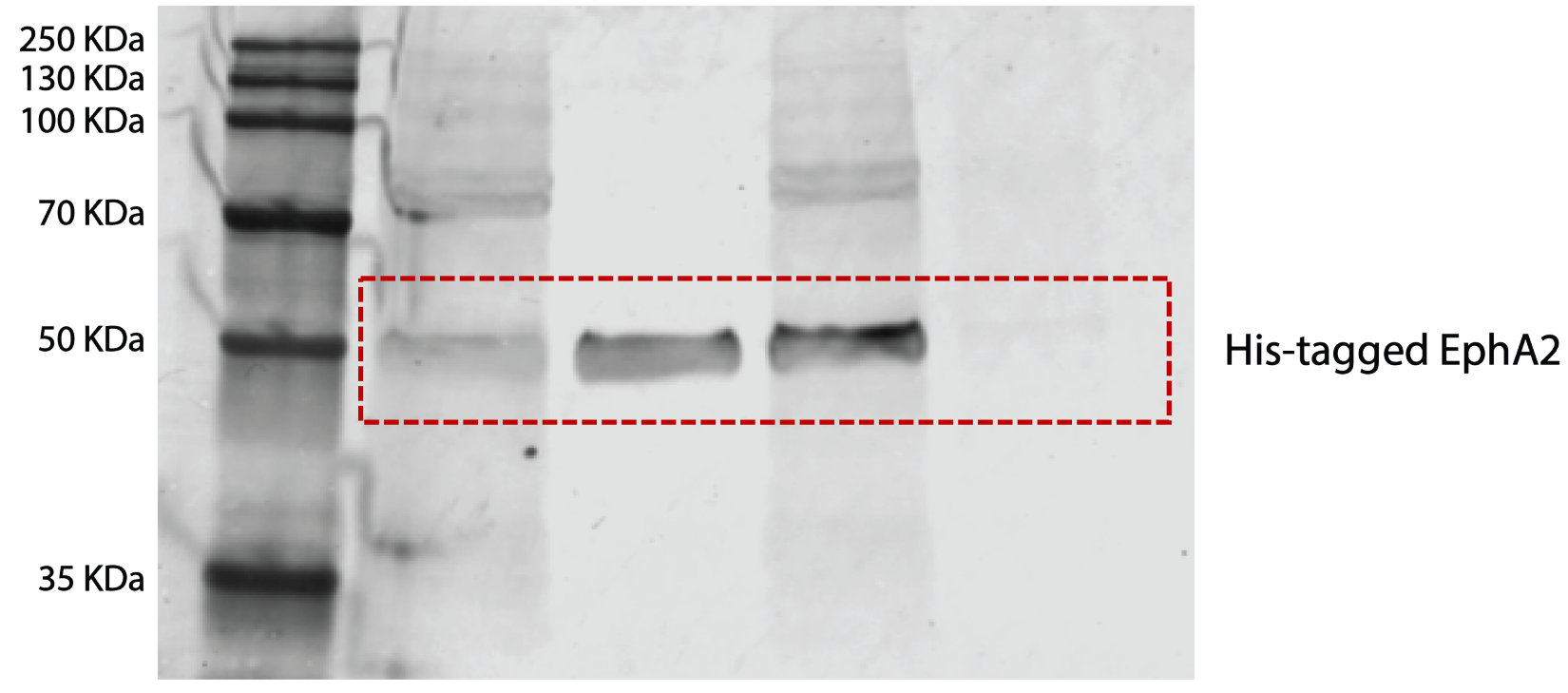

Immunoblots for Figure S3C, S4C

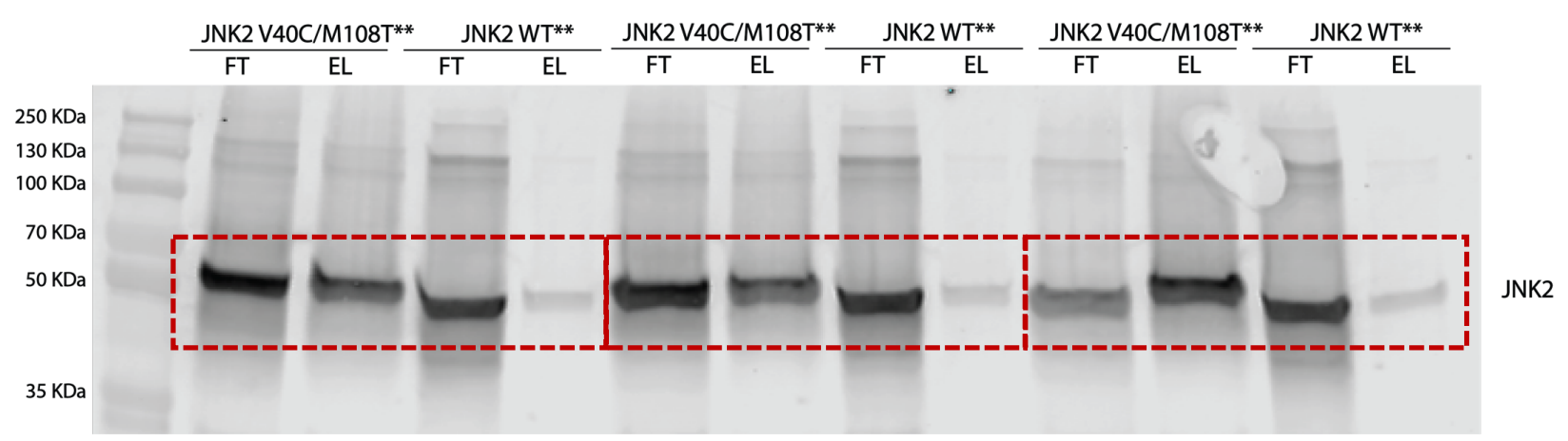

